November 2009

\title{
On the Inconsistency of Fayet-Iliopoulos Terms in Supergravity Theories
}

\author{
Keith R. Dienes ${ }^{1,2,3 *}$ and Brooks Thomas 3 沛 \\ 1 Physics Division, National Science Foundation, Arlington, VA 22230 USA \\ 2 Department of Physics, University of Maryland, College Park, MD 20742 USA \\ ${ }^{3}$ Department of Physics, University of Arizona, Tucson, AZ 85721 USA
}

\begin{abstract}
Motivated by recent discussions, we revisit the issue of whether globally supersymmetric theories with non-zero Fayet-Iliopoulos terms may be consistently coupled to supergravity. In particular, we examine claims that a fundamental inconsistency arises due to the conflicting requirements which are imposed on the $R$-symmetry properties of the theory by the supergravity framework. We also prove that certain kinds of Fayet-Iliopoulos contributions to the supercurrent supermultiplets of theories with non-zero Fayet-Iliopoulos terms fail to exist. A key feature of our discussion is an explicit comparison between results from the chiral (or "old minimal") and linear (or "new minimal") formulations of supergravity, and the effects within each of these formalisms that are induced by the presence of non-zero Fayet-Iliopoulos terms.
\end{abstract}

\footnotetext{
* E-mail address: dienes@physics.arizona.edu

$\dagger$ E-mail address: brooks@physics.arizona.edu

${ }^{\ddagger}$ Permanent address.
} 


\section{Introduction and overview}

Within the context of supersymmetric gauge theories, the $D$-field is truly unique. It is the only field component which is simultaneously invariant under both gauge and supersymmetry (SUSY) transformations, and thus the $D$-field can play roles in supersymmetric theories which are forbidden to other fields. One such role is in the introduction of the Fayet-Iliopoulos (FI) term [1]

$$
\xi D \in \xi \int d^{2} \theta d^{2} \bar{\theta} V
$$

where $\xi$ is the FI coefficient and $V$ is a real vector superfield. As is well known, FI terms figure prominently in the history of supersymmetry, and constitute one of only two ways of inducing spontaneous supersymmetry breaking. As a result, over the past two decades, FI terms have appeared in attempts at supersymmetric model-building which are too numerous to list.

Yet experience has shown that even within these contexts, FI terms face significant limitations. For example, they have very restrictive renormalization group flows. It also turns out that they almost never dominate in dynamical supersymmetry breaking. It also turns out to be very difficult to couple FI terms to supergravity, and progress in this area has a long and somewhat tortu(r)ous history (see, e.g., Refs. [2$25]$ ). As a result, despite the existence of a vast literature on this subject spanning several decades, the question of whether it is even consistent to couple theories with FI terms to gravity remains largely unresolved.

The first steps in attempting to generalize FI terms to local supersymmetry were undertaken in Ref. [3], where it was shown that the action for theories with such terms is invariant under a set of transformations which combines $U(1)$ gauge transformations with local chiral rotations of the supergravity fields. This picture was further refined in Refs. 8, 12], where it was shown that the presence of an FI term in supergravity results in a mixing between the $U(1)_{\mathrm{FI}}$ gauge symmetry and $U(1)_{R}$ rotations, leaving behind only a single $U(1)$ invariance which is a linear combination of the two original symmetries. Following this work, a number of investigators then endeavored to couple such a theory to matter fields in a consistent, gauge-invariant manner. Because the presence of the FI term effectively gauges the global $U(1)_{R}$ symmetry, any theory involving an FI term would have to satisfy an additional set of anomaly-cancellation conditions involving $U(1)_{R}$. Unfortunately, these conditions turn out to be quite stringent. In Ref. [20, for example, it was found that these conditions are not satisfied in the MSSM or in the simplest extensions thereof. While there do exist rather baroque models (e.g., models with flavor-dependent $R$-charges) which satisfy these constraints, such models tend to be riddled with other undesirable traits, such as rapid proton-decay rates or a broken $S U(3)$ color group. Subsequent investigations 22$]$ yielded similar results, and while such models have not been ruled out, phenomenologically successful ones are not easy to construct. 
But this is not the only difficulty. Even if a phenomenologically-consistent model could be realized, a further problem arises because the charge shifts of the sort required to preserve gauge invariance in FI models have been shown to be inconsistent with Dirac quantization in the presence of magnetic monopoles [16]. As a result, no consistent and phenomenologically viable models with FI terms are known to exist, either in field theory or in string theory. Taken together, these considerations have even led to speculations that fundamental FI terms may be incompatible with supergravity altogether.

In a recent paper [25], an explanation was proposed for these difficulties: although the FI term is indeed invariant under supersymmetry transformations as well as the regular abelian gauge transformations that remain after passing to Wess-Zumino gauge, the FI term breaks the invariance of the theory with respect to the larger, full set of abelian gauge transformations that are required in a fully supersymmetric context. Specifically, the supercurrent and energy-momentum tensor fail to be fully gauge-invariant when an FI term of the form in Eq. (1.1) is added to the theory. Moreover, in the presence of an FI term, the associated $R$-current also fails to be gauge invariant, even after the truncation to Wess-Zumino gauge.

In principle, these results would have enormous consequences [25]. For example, if a supersymmetric field theory has no FI term at high scales, then no FI term can be generated at lower scales. These results would also imply that FI terms cannot be generated either perturbatively or non-perturbatively. Finally, these results would imply, once and for all, that all supergravity theories must have vanishing FI terms. Thus, if true, the results in Ref. [25] would truly amount to a death penalty for FI terms. While FI terms might still arise in certain limited contexts (e.g., string theories whose particle spectra exhibit FI charges with non-vanishing traces), their role in most of supersymmetric particle physics would be seriously curtailed.

This paper is devoted to developing a more complete understanding of these results. In particular, because the $D$-field does not carry an $R$-charge, an FI term cannot be responsible for the breaking of the $R$-symmetry that would otherwise exist in a superconformal theory. Yet the results which are found in Ref. 25] explicitly break this $R$-symmetry. Similarly, the FI term, by itself, preserves the Fayet-Iliopoulos gauge symmetry. Yet the results which are found in Ref. [25] explicitly break this gauge symmetry - indeed, this is the primary point of Ref. [25].

The reason for these apparent inconsistencies is that the results of Ref. [25] were implicitly derived using what is known as the "chiral" (or "old minimal") formulation of supergravity [5]. Indeed, one of the subtleties associated with making supersymmetry local is that there exist several different formulations for off-shell supergravity. These differ from each other in the auxiliary-field content of the supergravity multiplet, and as a result they also differ from each other in their formulations of the supercurrent supermultiplet to which the supergravity multiplet must couple. However, even the classical symmetry properties of a given theory differ from one formulation to the next. As we will show, the gauge-non-invariance of the supercurrent 
highlighted in Ref. 25] is such a formalism-dependent property. Indeed, the chiral formalism is well known to implicitly break not only the $U(1)_{\text {FI }}$ gauge symmetry (if originally present), but also $R$-symmetry (if originally present). This then explains the results found in Ref. [25].

There do, however, exist other formalisms in which the supercurrent remains gauge invariant, and in which $R$-symmetry is explicitly preserved. One such formulation is the so-called "linear" (or "new minimal") formalism [10]. This formalism has the advantage of manifestly preserving the symmetries of the original theory from the outset, and thus does not lead to spurious broken-symmetry effects. One natural question, then, is to determine the extent to which the conclusions of Ref. [25] continue to apply, even within a gauge-invariant formalism such as the linear formalism.

In this paper, we shall therefore undertake a general analysis of the supercurrent supermultiplet and general symmetry structures of theories with non-zero FI terms. Throughout our analysis, we shall bear in mind the special role played by the fact that FI terms, by themselves, preserve $R$-symmetry. Other than this, we shall make no special assumptions about the theory in question. A key feature of our discussion will be an explicit comparison between results from the chiral and linear formulations of supergravity, and the effects within each of these formalisms that are induced by the presence of non-zero Fayet-Iliopoulos terms. Indeed, one of our results will be to prove that certain kinds of Fayet-Iliopoulos contributions to the supercurrent supermultiplets of theories with non-zero Fayet-Iliopoulos terms fail to exist.

Unfortunately, the literature on supersymmetry and supergravity is notoriously plagued with a plethora of different notations and conventions. Moreover, as mentioned above, there also exist a variety of different formalisms that can be used for describing what is often ultimately the same physics, and one of the goals of this paper is to provide an explicit comparison between results derived using the chiral formalism and those derived using the linear formalism. We have therefore attempted to write this paper so that our discussion and analysis is as streamlined and as completely self-contained as possible.

In Sect. 2, we review some basic facts about FI terms, establishing our notation and conventions along the way. In Sect. 3, we then outline some basic facts about the supercurrent supermultiplet and discuss the various forms which it may take in supersymmetric theories which do not respect the full set of symmetries of the superconformal group. In Sect. 4, we review the chiral-compensator formalism, paying special attention to the issues that arise in the presence of a non-zero FI term. We show, in particular, how this formalism allows us to obtain one version of the FI supercurrent, and we prove that certain $R$-symmetric FI contributions to the overall supercurrent supermultiplet do not exist in the chiral formalism. We also review the arguments of Ref. [25] which demonstrate that the breaking of $U(1)_{\text {FI }}$ gauge symmetry in this formalism directly leads to the appearance of an extra global symmetry, implying that such a theory cannot be consistently coupled to supergravity in the chiral formalism. In Sect. 5, we then turn our attention to the linear-compensator 
formalism. In marked contrast to the chiral-compensator formalism, this formulation has the advantage of maintaining $U(1)_{\mathrm{FI}}$ gauge invariance throughout. We again focus on issues that arise in the presence of a non-zero FI term, and demonstrate once again that certain FI contributions to the overall supercurrent supermultiplet do not exist. Finally, in Sect. 6, we discuss how the chiral and linear formalisms can ultimately be related to each other through a duality transformation in theories that respect $R$-symmetry, such as those that might include non-zero FI terms. We also show how the supercurrent supermultiplets in the two formalisms can be related to each other, and discuss the symmetry properties of the theories that emerge in the linear formalism in the presence of a non-zero FI term.

\section{The Fayet-Iliopoulos term: Basic facts}

We begin, for completeness, by recording some standard result: $*$ concerning FI terms. This material is completely standard and can be found in almost any textbook on elementary supersymmetry. As such, our goal in repeating it here is primarily to establish our notation and conventions, as well as to have it readily available for later use.

We begin by recalling that a general vector superfield $V$ can be expanded in the form

$$
\begin{aligned}
V=C & +i \theta \chi-i \bar{\theta} \bar{\chi}+\frac{i}{2} \theta \theta(M+i N)-\frac{i}{2} \overline{\theta \theta}(M-i N) \\
& -\theta \sigma^{\mu} \bar{\theta} A_{\mu}+i \theta \theta \bar{\theta}\left(\bar{\lambda}+\frac{i}{2} \bar{\sigma}^{\mu} \partial_{\mu} \chi\right)-i \overline{\theta \theta} \theta\left(\lambda+\frac{i}{2} \sigma^{\mu} \partial_{\mu} \bar{\chi}\right) \\
& +\frac{1}{2} \theta \theta \overline{\theta \theta}\left(D+\frac{1}{2} \square C\right)
\end{aligned}
$$

where $C, D, M, N$, and $A_{\mu}$ are all real, and where $\square \equiv \partial_{\mu} \partial^{\mu}$. Under a supersymmetry transformation with parameter $\epsilon$, these fields transform according to

$$
\begin{aligned}
\delta_{\epsilon} C & =i \epsilon \chi-i \overline{\epsilon \chi} \\
\delta_{\epsilon} \chi_{\alpha} & =\epsilon_{\alpha}(M+i N)+\left(\sigma^{\mu} \bar{\epsilon}\right)_{\alpha} \partial_{\mu} C+i\left(\sigma^{\mu} \bar{\epsilon}\right)_{\alpha} A_{\mu} \\
\delta_{\epsilon}(M+i N) & =2 i \overline{\epsilon \sigma^{\mu}} \partial_{\mu} \chi+2 \bar{\lambda} \bar{\epsilon} \\
\delta_{\epsilon} A^{\mu} & =i \overline{\epsilon \sigma} \bar{\sigma}^{\mu} \lambda+i \epsilon \sigma^{\mu} \bar{\lambda}+\epsilon \partial^{\mu} \chi+\bar{\epsilon} \partial^{\mu} \bar{\chi} \\
\delta_{\epsilon} \lambda_{\alpha} & =i \epsilon_{\alpha} D+\frac{1}{2}\left(\sigma^{\mu} \bar{\sigma}^{\nu} \epsilon\right)_{\alpha} F_{\mu \nu} \\
\delta_{\epsilon} D & =-\epsilon \sigma^{\mu} \partial_{\mu} \bar{\lambda}+\overline{\epsilon \sigma}^{\mu} \partial_{\mu} \lambda,
\end{aligned}
$$

${ }^{*}$ Here and throughout this paper, we shall follow the notation and conventions of Ref. 26. exactly. We shall also use the phrase " $R$-symmetry" to refer to a generic $\theta$-rotation, while we shall let $R_{5}$ denote the specific chiral $\theta$-rotation whose generator appears in the $\mathcal{N}=1$ supersymmetry algebra. 
where $F_{\mu \nu} \equiv \partial_{\mu} A_{\nu}-\partial_{\nu} A_{\mu}$. We observe from these results that only the $D$-field transforms into a total derivative.

Let us also recall the properties of these fields under $U(1)$ gauge transformations. In a supersymmetric theory, a $U(1)$ gauge transformation of the vector superfield $V$ generalizes to take the form

$$
V \rightarrow V+\Phi+\Phi^{\dagger}
$$

where $\Phi$ is a chiral superfield. For the individual component fields within $V$, this leads to the gauge transformations

$$
\begin{aligned}
\delta C & =\phi+\phi^{*} \\
\delta \chi & =-i \sqrt{2} \psi \\
\delta(M+i N) & =-2 i F \\
\delta A_{\mu} & =-i \partial_{\mu}\left(\phi-\phi^{*}\right) \\
\delta \lambda & =0 \\
\delta D & =0
\end{aligned}
$$

where $\phi, \psi$, and $F$ are the component fields within $\Phi$. The transformation for the vector field $A_{\mu}$ is the usual $U(1)$ gauge transformation, while the remaining transformations are associated with the generalizations of $U(1)$ gauge symmetry in a supersymmetric context. Passing to Wess-Zumino gauge amounts to fixing these remaining gauge transformations in such a way that $C, \chi, M$, and $N$ all vanish; this then leaves behind only the ordinary $U(1)$ gauge transformation associated with $A_{\mu}$. For complete generality, we shall avoid restricting to Wess-Zumino gauge and retain all fields in our theory. However, we shall continue to distinguish between the traditional $U(1)$ gauge symmetry associated with $A_{\mu}$, and the fuller, more general gauge symmetry which is additionally associated with the transformations of $C, \chi, M$, and $N$. When needed, we shall refer to these gauge symmetries as "little" and "big" respectively.

We thus see that $\lambda$ and $D$ are fully gauge invariant: their values are unaltered under the most general gauge transformations associated with all of the degrees of freedom within $\Phi$. By contrast, the fields $C, \chi, M$, and $N$ are invariant only under the traditional $U(1)$ gauge transformations associated with $A_{\mu}$ that survive the truncation to Wess-Zumino gauge. Finally, as expected, the vector field $A_{\mu}$ is not invariant under any of these gauge transformations.

Given these results, we can now analyze the transformation properties of the FI action

$$
\mathcal{S}_{\mathrm{FI}}=\int d^{4} x \mathcal{L}_{\mathrm{FI}} \quad \text { where } \quad \mathcal{L}_{\mathrm{FI}} \equiv 2 \xi \int d^{2} \theta d^{2} \bar{\theta} V=\xi\left(D+\frac{1}{2} \square C\right) .
$$

Under supersymmetry transformations, we see that both the $D$-term and the $C$ term transform into total derivatives, and these vanish under the spacetime integral $\int d^{4} x$. Thus, the FI Lagrangian is not supersymmetry invariant, but the FI action 


\begin{tabular}{||c||c|c|c||}
\hline \hline & SUSY & traditional $U(1)$ & full gauge invariance \\
\hline FI Lagrangian w/o $C$-term & no & yes & yes \\
FI action w/o $C$-term & yes & yes & yes \\
\hline FI Lagrangian w/ $C$-term & no & yes & no \\
FI action w/ $C$-term & yes & yes & yes \\
\hline \hline
\end{tabular}

Table 1: Invariances of the FI Lagrangian and FI action under supersymmetry transformations, under the traditional $U(1)$ gauge transformations that survive the truncation to Wess-Zumino gauge, and under the full gauge transformations associated with Eq. (2.3). We list results for the case in which the (total-derivative) $C$-term is dropped from Eq. (2.5) as well as the case in which it is retained.

is. By contrast, the situation regarding gauge invariance is a bit more subtle. Under the traditional $U(1)$ gauge symmetry (i.e., the remnant of the full gauge symmetry that survives the truncation to Wess-Zumino gauge), both the $D$-term and $C$-term are gauge invariant. As a result, both the FI Lagrangian and FI action are invariant under the traditional $U(1)$ gauge symmetry. However, under the full gauge symmetry associated with Eq. (2.3), the $D$-term is invariant while the $C$-term is not. Yet, even in this case, the $C$-term within Eq. (2.5) transforms into a total derivative. Thus, the FI action is actually invariant under the full gauge symmetry associated with Eq. (2.3) even though the FI Lagrangian is not. These transformation properties for the FI term are summarized in Table 1.

\section{Three possible structures for the supercurrent supermul- tiplet: A quick review}

In this section, we review the emergence and constraints that govern the supercurrent supermultiplet. We discuss these constraints in both the chiral and linear formalisms, as well as the relations between them.

\subsection{The supercurrent supermultiplet}

The $\mathcal{N}=1$ supersymmetry algebra is the algebra associated with super-Poincaré symmetry. Each of these charges in this algebra is associated with a current, and these currents (through the Noether theorem) are associated with different symmetries that together constitute the super-Poincaré group.

In this paper, we shall be concerned with three of these currents: the $R_{5}$-symmetry current $j_{\mu}^{(5)}$, associated with the chiral $R_{5}$-variations (phase rotations) of $\theta$ and $\bar{\theta}$; the supercurrent $j_{\mu \alpha}$, associated with supersymmetry transformations; and the energy-

momentum tensor $T_{\mu \nu}$, associated with spacetime translations. Each of these may be calculated in the usual way, through the Noether theorem; since equations of motion 
are used in the Noether derivation, such currents are intrinsically on-shell objects.

Ultimately, however, these currents are not physical: the only parts which are physical are their divergences $\partial^{\mu} j_{\mu}^{(5)}, \partial^{\mu} j_{\mu \alpha}$, and $\partial^{\mu} T_{\mu \nu}$ (indicating whether or not these symmetries are preserved), and their associated charges (i.e., the spatial integrals of their $\mu=0$ components). As a result, these currents themselves are not unique, and each may be modified in a variety of ways through the addition of socalled "improvement" terms which do do not change the physical properties of the theory in question. Thus, for any current $j_{\mu}$, an improvement term may affect the value of neither $\partial^{\mu} j_{\mu}$ nor $Q \equiv \int d^{3} x j_{0}$. It then follows that if a particular theory has a Noether current corresponding to an unbroken symmetry, i.e., if $\partial^{\mu} j_{\mu}=0$, then no improvement term can induce a non-zero value for $\partial^{\mu} j_{\mu}$. Improvement terms can, however, can be useful for making certain properties of a theory manifest. For example, in the case of abelian gauge theories, it is well known that the Noether procedure does not always yield an energy-momentum tensor $T_{\mu \nu}$ which is gauge invariant or symmetric in its indices. However, there exist improvement terms which can fix both of these difficulties. Likewise, in supersymmetric theories involving Kähler potentials, Kähler transformations will also modify the currents by altering the forms of these improvement terms.

In Ref. [2], it was shown that the $R_{5}$-current $j_{\mu}^{(5)}$, the supercurrent $j_{\mu \alpha}$, and the energy-momentum tensor $T_{\mu \nu}$ - all suitably improved - can be related to the lowestlying components of a real supermultiplet, the so-called supercurrent superfield. This fact is ultimately a consequence of the structure of the underlying super-Poincaré algebra which relates the charges associated with these currents. In general, the supercurrent supermultiplet is a real vector superfield which we may write in the form

$$
\begin{aligned}
J_{\mu}=C_{\mu} & +i \theta \chi_{\mu}-i \bar{\theta} \bar{\chi}_{\mu}+\frac{i}{2} \theta \theta\left(M_{\mu}+i N_{\mu}\right)-\frac{i}{2} \overline{\theta \theta}\left(M_{\mu}-i N_{\mu}\right) \\
& -\theta \sigma^{\nu} \bar{\theta} \hat{T}_{\nu \mu}+i \theta \theta \bar{\theta}\left(\bar{\lambda}_{\mu}+\frac{i}{2} \bar{\sigma}^{\nu} \partial_{\nu} \chi_{\mu}\right)-i \overline{\theta \theta} \theta\left(\lambda_{\mu}+\frac{i}{2} \sigma^{\nu} \partial_{\nu} \bar{\chi}_{\mu}\right) \\
& +\frac{1}{2} \theta \theta \overline{\theta \theta}\left(D_{\mu}+\frac{1}{2} \square C_{\mu}\right)
\end{aligned}
$$

where $C_{\mu}, D_{\mu}, M_{\mu}, N_{\nu}$, and $\hat{T}_{\nu \mu}$ are all real. Note that this form is completely analogous to that in Eq. (2.1) except that all fields now carry an additional Lorentz index. Consequently, under supersymmetry transformations, the components in Eq. (3.1) mix according to the relations in Eq. (2.2), suitably extended with an extra Lorentz index:

$$
\begin{aligned}
\delta_{\epsilon} C_{\mu} & =i \epsilon \chi_{\mu}-i \overline{\epsilon \chi}_{\mu} \\
\delta_{\epsilon} \chi_{\mu \alpha} & =\epsilon_{\alpha}\left(M_{\mu}+i N_{\mu}\right)+\left(\sigma^{\nu} \bar{\epsilon}\right)_{\alpha}\left(\partial_{\nu} C_{\mu}+i \hat{T}_{\nu \mu}\right) \\
\delta_{\epsilon}\left(M_{\mu}+i N_{\mu}\right) & =2 i \overline{\epsilon \sigma}^{\nu} \partial_{\nu} \chi_{\mu}+2 \bar{\epsilon} \bar{\lambda}_{\mu} \\
\delta_{\epsilon} \hat{T}_{\nu \mu} & =i \overline{\epsilon \sigma}_{\nu} \lambda_{\mu}+i \epsilon \sigma_{\nu} \bar{\lambda}_{\mu}+\epsilon \partial_{\nu} \chi_{\mu}+\bar{\epsilon} \partial_{\nu} \bar{\chi}_{\mu}
\end{aligned}
$$




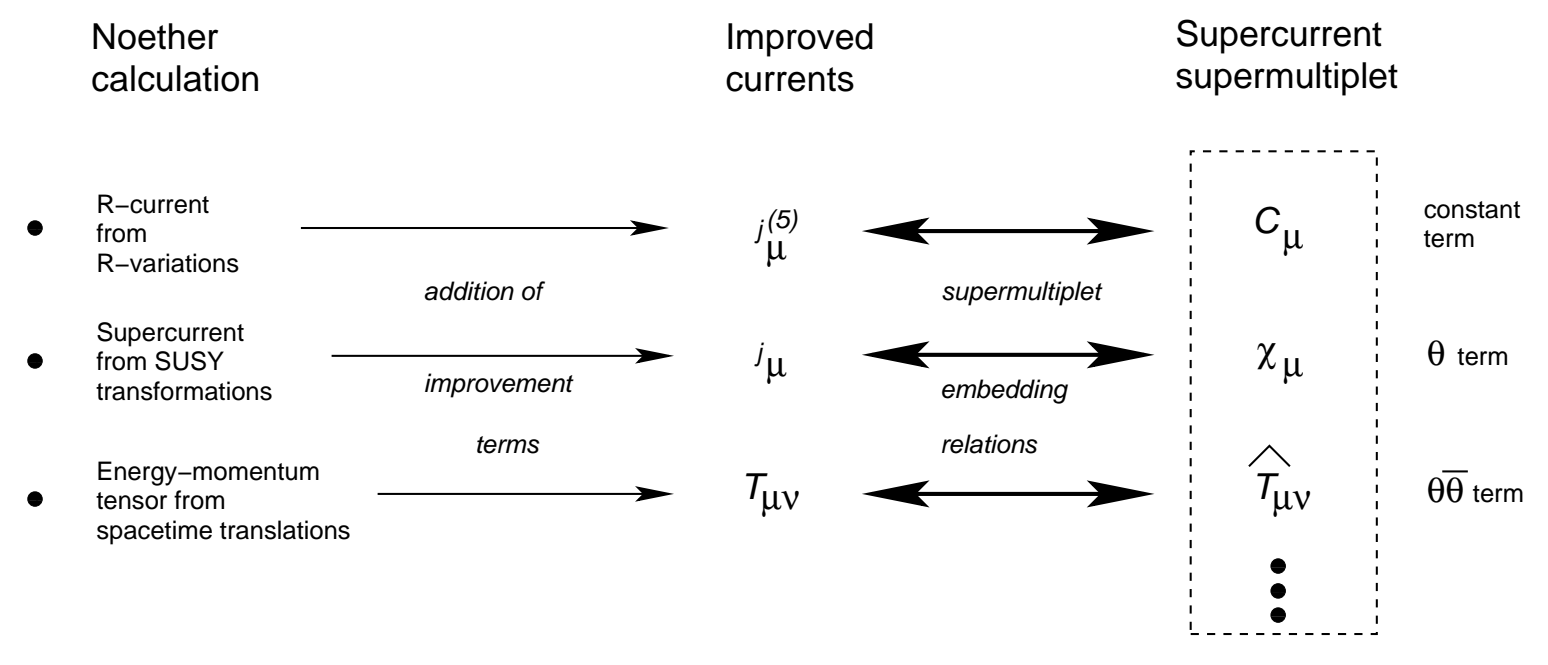

Figure 1: The general relation between the individual Noether currents and the supercurrent supermultiplet. While the Noether currents follow directly from their associated symmetries, they can each be modified through the addition of appropriate so-called "improvement" terms which preserve their divergences and charges. When suitably improved, these currents can then be mapped into the lowest components of a single supercurrent superfield through mapping relations which depend on the particular constraints that the supercurrent supermultiplet is assumed to obey.

$$
\begin{aligned}
\delta_{\epsilon} \lambda_{\mu \alpha} & =i \epsilon_{\alpha} D_{\mu}+\frac{1}{2}\left(\sigma^{\rho} \bar{\sigma}^{\sigma} \epsilon\right)_{\alpha}\left(\partial_{\rho} \hat{T}_{\sigma \mu}-\partial_{\sigma} \hat{T}_{\rho \mu}\right) \\
\delta_{\epsilon} D_{\mu} & =-\epsilon \sigma^{\nu} \partial_{\nu} \bar{\lambda}_{\mu}+\overline{\epsilon \sigma}^{\nu} \partial_{\nu} \lambda_{\mu} .
\end{aligned}
$$

The fields $C_{\mu}, \chi_{\mu \alpha}$, and $\hat{T}_{\nu \mu}$ are directly related to the improved versions of $j_{\mu}^{(5)}$, $j_{\mu \alpha}$, and $T_{\mu \nu}$ respectively. In fact, we can immediately identify $C_{\mu}=j_{\mu}^{(5)}$, but the precise mappings between $\chi_{\mu \alpha}$ and $j_{\mu \alpha}$, and between $\hat{T}_{\nu \mu}$ and $T_{\mu \nu}$, depend on the particular constraint equations satisfied by the supercurrent supermultiplet $J_{\mu}$ in Eq. (3.1). These in turn depend on the precise supermultiplet structure of the superconformal anomalies in the theory. Several different formalisms for approaching this issue exist, as we shall discuss in Sect. 3.2. This situation is schematically depicted in Fig. 1.

\subsection{Constraints on supercurrent supermultiplets in the superconformal, chiral, and linear formalisms: A review and comparison}

In this section, we review the forms that the supercurrent supermultiplet is expected to take in three different cases. These cases are ultimately distinguished by whether superconformal symmetry and $R_{5}$-symmetry are either separately preserved or broken. While additional cases may also exist [27, 28], these three cases represent the "minimal" constructions and will be the primary focus of this paper.

In general, there are three quantities which are relevant to determining the precise 
superconformal anomaly structure of a given theory: $\partial^{\mu} j_{\mu}^{(5)}, \bar{\sigma}^{\mu} j_{\mu \alpha}$, and $T \equiv T_{\mu}^{\mu}$. In a theory with unbroken superconformal invariance, all three of these quantities vanish, and the supercurrent supermultiplet satisfies the constraint equation

$$
\bar{D}^{\dot{\alpha}} J_{\alpha \dot{\alpha}}=0
$$

where $D_{\alpha}$ and $\bar{D}_{\dot{\alpha}}$ are the standard chiral covariant derivatives

$$
\begin{aligned}
D_{\alpha} & \equiv \frac{\partial}{\partial \theta^{\alpha}}+i\left(\sigma^{\mu} \bar{\theta}\right)_{\alpha} \partial_{\mu} \\
\bar{D}_{\dot{\alpha}} & \equiv-\frac{\partial}{\partial \bar{\theta}^{\dot{\alpha}}}-i\left(\theta \sigma^{\mu}\right)_{\dot{\alpha}} \partial_{\mu}
\end{aligned}
$$

and where

$$
J_{\alpha \dot{\alpha}} \equiv \sigma_{\alpha \dot{\alpha}}^{\mu} J_{\mu} .
$$

These equations therefore describe the superconformal case. Note that these constraints do not define $J_{\alpha \dot{\alpha}}$ uniquely; instead, $J_{\alpha \dot{\alpha}}$ is defined only up to the addition of real superfield improvement terms which are annihilated by $\bar{D}^{\dot{\alpha}}$. This is related to the fact that the supercurrent supermultiplet $J_{\alpha \dot{\alpha}}$ is not a physical object; indeed, only its divergence, charge, and associated anomalies are of relevance.

By contrast, in a general theory in which the superconformal symmetry is maximally broken, all three of the anomalies accrue non-zero values and fill out a chiral multiplet. In such a case, the supercurrent supermultiplet satisfies a constraint equation of the form

$$
\bar{D}^{\dot{\alpha}} J_{\alpha \dot{\alpha}}=D_{\alpha} S
$$

where $S$ is a chiral multiplet. Eq. (3.6) defines the chiral case. In this case, $J_{\alpha \dot{\alpha}}$ is defined up to the addition of real superfield improvement terms which, when acted on by $\bar{D}^{\dot{\alpha}}$, take the form $D_{\alpha} X$, where $X$ is a chiral superfield. Such improvement terms can be generated through Kähler transformations.

However, it is possible for a theory to break superconformal symmetry and yet preserve $R_{5}$-symmetry. In such a case, $\partial^{\mu} j_{\mu}^{(5)}$ vanishes while $\bar{\sigma}^{\mu} j_{\mu \alpha}$ and $T$ accrue non-zero values and together form a so-called linear multiplet $*$ The supercurrent supermultiplet then satisfies a constraint equation of the form

$$
\bar{D}^{\dot{\alpha}} J_{\alpha \dot{\alpha}}=L_{\alpha}
$$

where $L_{\alpha}$ is a chiral multiplet satisfying

$$
\bar{D}_{\dot{\beta}} L_{\alpha}=0 \quad \text { and } \quad D^{\alpha} L_{\alpha}=\bar{D}_{\dot{\alpha}} \bar{L}^{\dot{\alpha}} .
$$

${ }^{*}$ Recall that a linear multiplet, like a chiral multiplet, is an irreducible representation of the supersymmetry algebra contained within a vector multiplet $T$. Specifically, a linear multiplet can be realized as a vector multiplet $T$ which additionally satisfies the constraints $D^{\alpha} D_{\alpha} T=\bar{D}_{\dot{\alpha}} \bar{D}^{\dot{\alpha}} T=0$. Equivalently, a linear multiplet can be obtained from a general vector multiplet $T$ by setting the $\theta \theta$ and $\overline{\theta \theta}$ components to zero and imposing the reality condition $T^{\dagger}=T$. 
In other words, despite its name, $L_{\alpha}$ is structurally identical to the gauge multiplet $W_{\alpha}=\left(\lambda, F_{\mu \nu}, D\right)$, where $F_{\nu \mu}=-F_{\mu \nu}$. We will henceforth denote the components of $L_{\alpha}$ as $L_{\alpha}=\left(\Lambda, \Phi_{\mu \nu}, \Delta\right)$, where similarly $\Phi_{\nu \mu}=-\Phi_{\mu \nu}$. Eq. (3.7) then defines the linear case. Indeed, as we shall shortly demonstrate, Eq. (3.8) implies that $J_{\alpha \dot{\alpha}}$ is itself a linear multiplet.

In Table 2, we indicate the explicit supercurrent supermultiplet structures that emerge in each of these three cases. In the first two columns, we provide the explicit term-by-term expansion of the superfield $\bar{D}^{\dot{\alpha}} J_{\alpha \dot{\alpha}}$, where

$$
\bar{D}^{\dot{\alpha}} J_{\alpha \dot{\alpha}}=-\epsilon^{\dot{\alpha} \dot{\beta}} \sigma_{\alpha \dot{\alpha}}^{\mu} \frac{\partial}{\partial \bar{\theta}^{\dot{\beta}}} J_{\mu}+i\left(\sigma^{\mu} \bar{\sigma}^{\nu} \theta\right)_{\alpha} \partial_{\nu} J_{\mu}
$$

with $J_{\mu}$ is given in Eq. (3.1). In the remaining columns, we also provide the corresponding term-by-term expansion of the different superfields to which $\bar{D}^{\dot{\alpha}} J_{\alpha \dot{\alpha}}$ must be equated in each of these three cases. In general, equating the coefficients of these expansions yields a set of constraint equations for the fields that appear in these coefficients. Note that in extracting the constraint equations for these coefficients, we have made use of two facts which apply when the coefficients are scalars in spinor space:

$$
\begin{array}{rll}
\sigma^{\mu} A_{\mu}=0 & \Longrightarrow & A_{\mu}=0 \\
\sigma^{\mu \nu}\left(A_{\mu \nu}+i B_{\mu \nu}\right)=0 & \Longrightarrow \quad A^{\mu \nu}=\frac{1}{2} \epsilon^{\mu \nu \rho \sigma} B_{\rho \sigma}
\end{array}
$$

where in the second line $A_{\mu \nu}$ and $B_{\mu \nu}$ are each taken to be real and anti-symmetric in $(\mu, \nu)$ indices.

Solving the resulting constraint equations in each case, we indeed find that our anomalies take the required vanishing or non-vanishing forms indicated at the bottom of Table 2. Moreover, given these solutions to the constraint equations, we can also determine which particular combinations of $\chi_{\mu}$ and $\hat{T}_{\mu \nu}$ are conserved and can thus correspond to the supercurrent $j_{\mu}$ and energy-momentum tensor $T_{\mu \nu}$, respectively. Our results are as follows. In the superconformal case, we find

$$
\left\{\begin{array}{l}
j_{\mu \alpha}=\chi_{\mu \alpha} \\
T_{\mu \nu}=-\frac{1}{4}\left(\hat{T}_{\mu \nu}+\hat{T}_{\nu \mu}\right)
\end{array}\right.
$$

while in the chiral case, we find

$$
\left\{\begin{array} { l } 
{ j _ { \mu \alpha } = \chi _ { \mu \alpha } + ( \sigma _ { \mu } \overline { \sigma } ^ { \nu } \chi _ { \nu } ) _ { \alpha } } \\
{ T _ { \mu \nu } = - \frac { 1 } { 4 } ( \hat { T } _ { \mu \nu } + \hat { T } _ { \nu \mu } - 2 g _ { \mu \nu } \hat { T } ) }
\end{array} \Longrightarrow \left\{\begin{array}{l}
\chi_{\mu \alpha}=j_{\mu \alpha}+\frac{1}{3}\left(\sigma_{\mu} \bar{\sigma}^{\nu} j_{\nu}\right)_{\alpha} \\
\left.\hat{T}_{\mu \nu}\right|_{\mathrm{symm}}=-2 T^{\mu \nu}+\frac{2}{3} g_{\mu \nu} T
\end{array}\right.\right.
$$

where $\left.\hat{T}_{\mu \nu}\right|_{\text {symm }}$ indicates the symmetric part of $\hat{T}_{\mu \nu}$. Note that Eq. (3.12) implies the anomaly relations $\bar{\sigma}^{\mu} j_{\mu}=-3 \bar{\sigma}^{\mu} \chi_{\mu}$ and $T=3 \hat{T} / 2$. However, in the linear case, we find

$$
\left\{\begin{array}{l}
j_{\mu \alpha}=\chi_{\mu \alpha} \\
T_{\mu \nu}=-\frac{1}{4}\left(\hat{T}_{\mu \nu}+\hat{T}_{\nu \mu}\right) .
\end{array}\right.
$$




\begin{tabular}{|c|c|c|c|c|}
\hline Term & $\bar{D}^{\dot{\alpha}} J_{\alpha \dot{\alpha}}$ & $\begin{array}{c}\text { super- } \\
\text { conformal }\end{array}$ & $\begin{array}{l}D_{\alpha} S, \text { where } \\
S:(\phi, \psi, F) \\
\end{array}$ & $\begin{array}{c}L_{\alpha}, \text { where } \\
L:\left(\Lambda, \Phi_{\mu \nu}, \Delta\right) \\
\end{array}$ \\
\hline no $\theta, \bar{\theta}$ & $-i\left(\sigma^{\mu} \bar{\chi}_{\mu}\right)_{\alpha}$ & 0 & $\sqrt{2} \psi_{\alpha}$ & $-i \Lambda_{\alpha}$ \\
\hline $\begin{array}{l}\theta_{\alpha}:(\operatorname{Re}) \\
\theta_{\alpha}:(\operatorname{Im})\end{array}$ & $\begin{array}{c}-\hat{T} \\
-i \partial^{\mu} C_{\mu}\end{array}$ & $\begin{array}{l}0 \\
0\end{array}$ & $\begin{array}{l}2 \operatorname{Re}(F) \\
2 \operatorname{Im}(F)\end{array}$ & $\begin{array}{l}\Delta \\
0\end{array}$ \\
\hline$\left(\sigma^{\mu \nu} \theta\right)_{\alpha}$ & $\begin{array}{c}-\left(\hat{T}_{\mu \nu}-\hat{T}_{\nu \mu}\right) \\
+\frac{1}{2} \epsilon_{\mu \nu \rho \sigma}\left(\partial^{\rho} C^{\sigma}-\partial^{\sigma} C^{\rho}\right)\end{array}$ & 0 & 0 & $\frac{1}{2} \epsilon_{\mu \nu \rho \sigma} \Phi^{\rho \sigma}$ \\
\hline $\begin{array}{l}\left(\sigma^{\mu} \bar{\theta}\right)_{\alpha}:(\operatorname{Re}) \\
\left(\sigma^{\mu} \bar{\theta}\right)_{\alpha}:(\operatorname{Im})\end{array}$ & $\begin{array}{l}-N_{\mu} \\
-i M_{\mu}\end{array}$ & $\begin{array}{l}0 \\
0\end{array}$ & $\begin{array}{l}-2 \partial_{\mu} \operatorname{Im}(\phi) \\
2 i \partial_{\mu} \operatorname{Re}(\phi)\end{array}$ & $\begin{array}{l}0 \\
0\end{array}$ \\
\hline$\theta \theta$ & $i\left(\sigma^{\mu} \bar{\lambda}_{\mu}\right)_{\alpha}$ & 0 & 0 & $\left(\sigma^{\mu} \partial_{\mu} \bar{\Lambda}\right)_{\alpha}$ \\
\hline$\overline{\theta \theta}$ & 0 & 0 & 0 & 0 \\
\hline$\left(\sigma^{\nu} \bar{\theta}\right)_{\gamma} \theta^{\beta}$ & $\begin{array}{c}-\delta_{\beta}^{\gamma}\left(\sigma^{\mu} \partial_{\nu} \bar{\chi}_{\mu}\right)_{\alpha} \\
-2 i \delta_{\alpha}^{\gamma} \lambda_{\nu \beta}+2 \delta_{\alpha}^{\gamma}\left(\sigma^{\mu} \partial_{\mu} \bar{\chi}_{\nu}\right)_{\beta}\end{array}$ & 0 & $\begin{array}{l}-\sqrt{2} i \delta_{\beta}^{\gamma} \partial_{\nu} \psi_{\alpha} \\
+2 \sqrt{2} i \partial_{\alpha}^{\gamma} \partial_{\nu} \psi_{\beta}\end{array}$ & $-\delta_{\beta}^{\gamma} \partial_{\nu} \Lambda_{\alpha}$ \\
\hline $\begin{array}{l}(\theta \theta)\left(\sigma^{\mu} \bar{\theta}\right)_{\alpha}:(\mathrm{Re}) \\
(\theta \theta)\left(\sigma^{\mu} \bar{\theta}\right)_{\alpha}:(\operatorname{Im})\end{array}$ & $\begin{array}{c}D_{\mu}+\frac{1}{2} \square C_{\mu}+\frac{1}{2} \epsilon_{\mu \nu \rho \sigma} \partial^{\nu} \hat{T}^{\rho \sigma} \\
-\frac{i}{2} \partial^{\nu}\left(\hat{T}_{\mu \nu}+\hat{T}_{\nu \mu}-g_{\mu \nu} \hat{T}\right)\end{array}$ & $\begin{array}{l}0 \\
0\end{array}$ & $\begin{array}{c}-\partial_{\mu} \operatorname{Im}(F) \\
i \partial_{\mu} \operatorname{Re}(F)\end{array}$ & $\begin{array}{l}\frac{1}{2} \partial^{\nu} \Phi_{\mu \nu} \\
-\frac{i}{2} \partial_{\mu} \Delta\end{array}$ \\
\hline $\begin{array}{l}(\overline{\theta \theta}) \theta_{\alpha}:(\mathrm{Re}) \\
(\overline{\theta \theta}) \theta_{\alpha}:(\operatorname{Im})\end{array}$ & $\begin{array}{c}-\frac{1}{2} \partial^{\mu} M_{\mu} \\
\frac{i}{2} \partial^{\mu} N_{\mu}\end{array}$ & $\begin{array}{l}0 \\
0\end{array}$ & $\begin{array}{l}\square \operatorname{Re}(\phi) \\
i \square \operatorname{Im}(\phi)\end{array}$ & $\begin{array}{l}0 \\
0\end{array}$ \\
\hline$(\overline{\theta \theta})\left(\sigma^{\mu \nu} \theta\right)_{\alpha}$ & $\begin{array}{c}-\frac{1}{2}\left(\partial_{\mu} M_{\nu}-\partial_{\nu} M_{\mu}\right) \\
-\frac{1}{4} \epsilon_{\mu \nu \rho \sigma}\left(\partial^{\rho} N^{\sigma}-\partial^{\sigma} N^{\rho}\right)\end{array}$ & 0 & 0 & 0 \\
\hline \multirow[t]{2}{*}{$\theta \theta \overline{\theta \theta}$} & $-\frac{1}{2}\left(\sigma^{\nu} \bar{\sigma}^{\mu} \partial_{\mu} \lambda_{\nu}\right)_{\alpha}+\frac{i}{4}\left(\sigma^{\mu} \square \bar{\chi}_{\mu}\right)_{\alpha}$ & 0 & $-\frac{1}{2 \sqrt{2}} \square \psi_{\alpha}$ & $-\frac{i}{4} \square \Lambda_{\alpha}$ \\
\hline & $\begin{aligned} & \text { superconformal }\left\{\begin{array}{l}\partial^{\mu} C_{\mu} \\
\text { anomalies : }\end{array}(\bar{\sigma} \chi)_{\alpha}\right. \\
& \hat{T}\end{aligned}$ & $\begin{array}{l}0 \\
0 \\
0\end{array}$ & $\begin{array}{l}-2 \operatorname{Im}(F) \\
\sqrt{2} i \psi_{\alpha} \\
-2 \operatorname{Re}(F)\end{array}$ & $\begin{array}{c}0 \\
\Lambda_{\alpha} \\
-\Delta\end{array}$ \\
\hline
\end{tabular}

Table 2: Three different structures for the supercurrent supermultiplet, corresponding to the unbroken superconformal case, the maximally broken (chiral) case, and the (linear) case in which superconformal symmetry is broken but $R_{5}$-symmetry is preserved. 
Thus, we see that the linear case resembles the superconformal case, even though the superconformal symmetry is broken and $\bar{\sigma}^{\mu} \chi_{\mu}$ and $T$ are both non-zero. Indeed, the critical feature that links these two cases is the fact that $R_{5}$-symmetry is unbroken.

Ultimately, in passing from global supersymmetry to local supersymmetry, these supercurrents are coupled to the supergravity multiplet. As a result, each of these formalisms leads to a different self-consistent formulation of off-shell supergravity with a unique auxiliary-field content. In the chiral formalism [5], for example, the complete supercurrent supermultiplet takes the form $\left(j_{\mu}^{(5)}, j_{\mu \alpha}, T_{\mu \nu}, P, Q\right)$ where $P$ and $Q$ are two additional scalar currents. This multiplet can therefore couple to a supergravity multiplet of the form $\left(b_{\mu}, \psi_{\mu \alpha}, e_{\mu}^{m}, M, N\right)$, where where $b_{\mu}$ is the connection field associated with local chiral rotations, where $\psi_{\mu \alpha}$ is the gravitino, where $e_{\mu}^{m}$ is the vielbein, and where $M$ and $N$ are additional real scalar degrees of freedom. Indeed, this supergravity multiplet is nothing but a particular truncation of the more general superconformal supergravity multiplet $\left(b_{\mu}, \psi_{\mu \alpha}, e_{\mu}^{m}, a_{\mu}\right)$ where $a_{\mu}$ is the connection field associated with local chiral dilatations. Thus, we see that the form of the supercurrent supermultiplet uniquely fixes the form of the corresponding supergravity supermultiplet.

By contrast, in the linear formalism (also occasionally called the "new" minimal formalism) [10], the full supercurrent supermultiplet has the structure of a linear multiplet $\left(C_{\mu}, \chi_{\mu \alpha},\left.\hat{T}_{\mu \nu}\right|_{\text {symm }},\left.\hat{T}_{\mu \nu}\right|_{\text {anti-symm }}\right)$. This then corresponds to a supergravity multiplet which can be written in the form $\left(b_{\mu}, \psi_{\mu \alpha}, e_{\mu}^{m}, a_{\mu \nu}\right)$, where $a_{\mu \nu}$ is an antisymmetric tensor. As we shall discuss in Sect. 5, this formalism can only be applied to $R_{5}$-invariant theories.

Clearly, the off-shell structures of the supergravity multiplet in the chiral and linear formalisms are quite different. However, it has been shown in Ref. [14] that although the linear and chiral formalisms are not generally equivalent, they become so at the classical level if the Lagrangian of the theory respects $R_{5}$-symmetry. This will be discussed in more detail in Sect. 6.1. In such a case, the corresponding supercurrent supermultiplets can be related to each other through the addition of appropriate improvement terms [7]. Their corresponding anomaly multiplets can also be related in a similar way. These relations will be discussed more fully in Sect. 6 .

Each of these formalisms has its advantages and disadvantages, as well as its range of validity in terms of the globally supersymmetric theories to which it may be applied. For example, the chiral formalism is applicable in a wide variety of contexts, including models with explicit $R_{5}$-symmetry breaking, though the superconformal gauge choice which renders it useful for calculation can also obscure the symmetry properties of the underlying theory, especially those associated with $R_{5}$-symmetries. By contrast, the linear formalism can only be applied to $R_{5}$-invariant theories. However, it has the advantage of manifesting the full $R_{5}$-invariance of such theories, which is obscured by superconformal gauge-fixing in the chiral formalism.

In the special case when the theory possesses an $R_{5}$-symmetry, these two for- 
malisms are related by a duality transformation. This will be discussed in Sect. 6 . Nevertheless, even for $R_{5}$-invariant theories, the representations of the supergravity multiplets in these two formalisms differ in many salient aspects. In the chiral formalism, for example, the usual form of the FI Lagrangian (i.e., the D-term within the vector superfield $\xi V$ ) is not gauge invariant. However, this can be remedied [8] by replacing the FI Lagrangian with the $D$-term associated with the superfield $\kappa^{-2} e^{\kappa^{2} \xi V}$ (with $\kappa^{2} \equiv M_{P}^{-2}$ ). In this case, gauge transformations of the form $V \rightarrow V^{\prime}=V+\Phi+\Phi^{\dagger}$, where $\Phi$ denotes a chiral superfield and $\Phi^{\dagger}$ its conjugate, can be compensated for by mandating that the fields of the supergravity multiplet transform in such a manner as to render the action invariant [12]. If the theory contains matter, the matter fields must also transform non-trivially under $U(1)$ gauge shifts in order to compensate for the requisite shifts in the supergravity fields. Consequently, the $U(1)$ charges of the matter fields of the theory are modified from their globally supersymmetric values [4]. One can show that a given action can only be made gauge invariant if the corresponding superpotential has an $R_{5}$-symmetry.

By contrast, the picture in the linear formalism is quite different. Here, the standard form of the FI Lagrangian - appropriately modified to include couplings between the fields in $V$ and the fields of the supergravity multiplet - is manifestly gauge invariant [11. The theory is $R_{5}$-invariant by assumption, and, as stated in the Introduction, the $U(1)$ invariance of the theory involves a combination of $U(1)_{\mathrm{FI}}$ gauge shifts and $U(1)_{R}$ rotations.

\subsection{Theories with unbroken $R_{5}$-symmetry}

Because $R_{5}$-symmetry is preserved in both the superconformal and linear cases, the supercurrent supermultiplet that emerges in the linear case shares many properties with the multiplet that emerges in the superconformal case. In particular, both supercurrent multiplets are truncations of the general supercurrent supermultiplet in Eq. (3.1) in which $M_{\mu}$ and $N_{\mu}$ vanish - i.e., they are linear multiplets. We can therefore probe their common structure by examining the general properties of supermultiplets in which these two components vanish. Consulting Eq. (3.2), we find that such a truncated multiplet can indeed be consistent with the supersymmetry algebra only if its remaining components satisfy the additional relations

$$
\begin{aligned}
\lambda_{\mu \alpha} & =-i\left(\sigma^{\nu} \partial_{\nu} \bar{\chi}_{\mu}\right)_{\alpha} \\
\partial^{\nu} \hat{T}_{\nu \mu} & =0 \\
D_{\mu} & =-\square C_{\mu} .
\end{aligned}
$$

Note, in particular, that the second equation in Eq. (3.14) is not a conservation law for $\hat{T}_{\nu \mu}$ because $\hat{T}_{\nu \mu}$ is not generally symmetric, and because a conservation law would be a statement about $\partial^{\mu} \hat{T}_{\nu \mu}$, not $\partial^{\nu} \hat{T}_{\nu \mu}$. Given $M_{\mu}=N_{\mu}=0$ and Eq. (3.14), the supersymmetry transformations in Eq. (3.2) then reduce to

$$
\delta_{\epsilon} C_{\mu}=i \epsilon \chi_{\mu}-i \overline{\epsilon \chi}_{\mu}
$$




$$
\begin{aligned}
\delta_{\epsilon} \chi_{\mu \alpha} & =\left(\sigma^{\nu} \bar{\epsilon}\right)_{\alpha}\left(\partial_{\nu} C_{\mu}+i \hat{T}_{\nu \mu}\right) \\
\delta_{\epsilon} \hat{T}_{\nu \mu} & =2 \overline{\epsilon \sigma}_{\nu \rho} \partial^{\rho} \bar{\chi}_{\mu}+2 \epsilon \sigma_{\nu \rho} \partial^{\rho} \chi_{\mu} .
\end{aligned}
$$

Several things are immediately apparent from Eq. (3.15). First, acting with $\partial^{\mu}$ on the first equation within Eq. (3.15), we see that

$$
\left(\partial^{\mu} C_{\mu}=0\right) \quad \Longrightarrow \quad\left(\partial^{\mu} \chi_{\mu}=0\right) \text {. }
$$

This explains why the conservation of $R_{5}$-symmetry and the identification $j_{\mu}^{(5)}=C_{\mu}$ together compel the identification $j_{\mu \alpha}=\chi_{\mu \alpha}$ for such multiplets, as already seen in Eqs. (3.11) and (3.13). Moreover, taking the trace of the third equation in Eq. (3.15), we find

$$
(\hat{T}=0) \quad \Longrightarrow \quad\left(\sigma^{\mu} \bar{\chi}_{\mu}=0\right) \text {. }
$$

Indeed, these results are intrinsic to the structure of any multiplet in which $M_{\mu}$ and $N_{\mu}$ vanish.

It is important to note that the intrinsic structure of such a multiplet does not, in and of itself, require that $\partial^{\mu} C_{\mu}=0$, nor that $\sigma^{\mu} \bar{\chi}_{\mu}=0$ or $\hat{T}=0$. Indeed, whether or not such additional constraints hold depends on whether we demand that such a multiplet $J_{\alpha \dot{\alpha}}$ additionally satisfy either the superconformal condition $\bar{D}^{\dot{\alpha}} J_{\alpha \dot{\alpha}}=0$ or the "linear" condition $\bar{D}^{\dot{\alpha}} J_{\alpha \dot{\alpha}}=L_{\alpha}$, where $L_{\alpha}$ satisfies the constraints in Eq. (3.8). Consulting Table 2, we find that imposing these additional conditions leads to the constraint $\partial^{\mu} C_{\mu}=0$ in both cases, while $\sigma^{\mu} \bar{\chi}_{\mu}$ and $\hat{T}$ are either both zero (in the superconformal case) or non-zero (in the linear case). Likewise, again consulting Table 2, we see that $\hat{T}_{\mu \nu}$ will be symmetric in the superconformal case, while it will not be symmetric in the linear case. In either case, however, the components $\left(C_{\mu}, \chi_{\mu}, \hat{T}_{\nu \mu}\right)$ are guaranteed to satisfy Eqs. (3.15), (3.16), and (3.17).

Thus far, we have been discussing the behavior of $R_{5}$-symmetric theories in the linear formalism. We have been concentrating on the linear formalism because we necessarily have $\partial^{\mu} C_{\mu}=0$ in the linear case, while we necessarily have $\partial^{\mu} C_{\mu} \neq 0$ in the chiral case. Indeed, we cannot have $\partial^{\mu} C_{\mu}=0$ within the chiral case unless $S=0$, and this only occurs for a superconformal theory. Thus, strictly speaking, an $R_{5}$-symmetric theory has a supercurrent supermultiplet which can only be described through the linear formalism.

Despite this, there can be situations in which we might wish to use the chiral formalism, even for theories in which $R_{5}$-symmetry is preserved but superconformal invariance is broken. Indeed, while the linear-case formalism incorporates this symmetry structure in the most manifest way, it may still be possible to adopt the chiral-case formalism in which this full symmetry structure is not manifest. Therefore, for an $R_{5}$-invariant theory, we have the possibility of constructing two distinct supercurrent supermultiplets, $J_{\alpha \dot{\alpha}}^{(L)}$ and $J_{\alpha \dot{\alpha}}^{(C)}$, the first of which satisfies $\bar{D}^{\dot{\alpha}} J_{\alpha \dot{\alpha}}^{(L)}=L_{\alpha}$ and the second of which satisfies $\bar{D}^{\dot{\alpha}} J_{\alpha \dot{\alpha}}^{(C)}=D_{\alpha} S$. We may interpret $J_{\alpha \dot{\alpha}}^{(L)}$ as the supercurrent supermultiplet, unique up to Kähler transformations, that may be formed 
from our original three Noether currents (corresponding to $R_{5}$-chiral rotations, supersymmetry transformations, and spacetime translations) through the addition of a specific set of improvement terms of each case. By contrast, we may interpret $J_{\alpha \dot{\alpha}}^{(C)}$ as an alternative supercurrent supermultiplet which can be formed for such a theory if we are willing to disregard any connection between $C_{\mu}$ and $j_{\mu}^{(5)}$, and only focus on embedding $j_{\mu \alpha}$ and $T_{\mu \nu}$ into a supercurrent superfield. Such an embedding is clearly different from the embedding that would occur within $J_{\alpha \dot{\alpha}}^{(L)}$, and corresponds to different improvement terms for $j_{\mu \alpha}$ and $T_{\mu \nu}$. Yet, for an $R_{5}$-invariant theory, $J_{\alpha \dot{\alpha}}^{(C)}$ represents an alternative supercurrent superfield that we may consider if we are willing to concentrate on $j_{\mu \alpha}$ and $T_{\mu \nu}$ only, and disregard any connection between $j_{\mu}^{(5)}$ (which is conserved) and $C_{\mu}$ (which is not conserved).

Clearly, for $R_{5}$-invariant theories, $J_{\alpha \dot{\alpha}}^{(C)}$ does not capture the full symmetry properties of the theory. Indeed, the bottom component of $J_{\alpha \dot{\alpha}}^{(C)}$ is not conserved and therefore cannot bear any relation to $j_{\mu}^{(5)}$, regardless of the addition of any possible improvement terms. Therefore, strictly speaking, the supercurrent supermultiplet $J_{\alpha \dot{\alpha}}^{(C)}$ does not correspond to the original $R_{5}$-symmetric theory in question. However, as we shall discuss in Sect. $4, J_{\alpha \dot{\alpha}}^{(C)}$ actually corresponds to a different theory which is closely related to our original $R_{5}$-symmetric theory. Thus, it is with this understanding that we shall loosely speak of an $R_{5}$-symmetric theory as having two possible supercurrent supermultiplets, $J_{\alpha \dot{\alpha}}^{(L)}$ and $J_{\alpha \dot{\alpha}}^{(C)}$.

\subsection{An example, both with and without an FI term}

As an example of these results, let us consider the specific pure-gauge $U(1)$ theory defined by the Lagrangian

$$
\begin{aligned}
\mathcal{L} & =\frac{1}{4}\left(\left.W^{\alpha} W_{\alpha}\right|_{\theta \theta}+\left.\bar{W}_{\dot{\alpha}} \bar{W}^{\dot{\alpha}}\right|_{\overline{\theta \theta}}\right) \\
& =-\frac{1}{4} F^{2}-\frac{i}{2} \lambda \sigma^{\mu}\left(\partial_{\mu} \bar{\lambda}\right)+\frac{i}{2}\left(\partial_{\mu} \lambda\right) \sigma^{\mu} \bar{\lambda}+\frac{1}{2} D^{2}
\end{aligned}
$$

where $W_{\alpha}$ is the gauge supermultiplet and $\square=\partial_{\mu} \partial^{\mu}$. The equations of motion for the different fields in this theory are then given by $\bar{D}_{\dot{\alpha}} \bar{W}^{\dot{\alpha}}=0$, i.e.,

$$
\partial_{\mu} F^{\mu \nu}=0, \quad \bar{\sigma}^{\mu} \partial_{\mu} \lambda=\sigma^{\mu} \partial_{\mu} \bar{\lambda}=0, \quad D=0,
$$

and the Noether currents corresponding to this theory are given by

$$
\begin{aligned}
j_{\mu}^{(5)}= & -\lambda \sigma_{\mu} \bar{\lambda} \\
j_{\mu \alpha}= & -i\left(F_{\mu \nu}+\tilde{F}_{\mu \nu}\right)\left(\sigma^{\nu} \bar{\lambda}\right)_{\alpha}-F_{\mu \nu} \partial^{\nu} \chi_{\alpha} \\
T_{\mu \nu}= & -\frac{1}{4} g_{\mu \nu} F^{2}+F_{\mu \rho} \partial_{\nu} A^{\rho} \\
& \quad+\frac{i}{2} \lambda \sigma_{\mu}\left(\partial_{\nu} \bar{\lambda}\right)-\frac{i}{2}\left(\partial_{\nu} \lambda\right) \sigma_{\mu} \bar{\lambda}+\frac{1}{2} g_{\mu \nu} D^{2}
\end{aligned}
$$


where $\tilde{F}^{\mu \nu} \equiv \frac{i}{2} \epsilon^{\mu \nu \lambda \sigma} F_{\lambda \sigma}$. Note that we may use the equations of motion in Eq. (3.19) in order to eliminate the $D$-term within $T_{\mu \nu}$ if we wish. Moreover, using the equations of motion, it is straightforward to verify that each of these currents is conserved, i.e.,

$$
\partial^{\mu} j_{\mu}^{(5)}=0, \quad \partial^{\mu} j_{\mu \alpha}=0, \quad \partial^{\mu} T_{\mu \nu}=0
$$

as guaranteed by the Noether construction.

At first glance, it may seem a bit surprising that the supercurrent and energymomentum tensor contain terms of the form $-F_{\mu \nu} \partial^{\nu} \chi_{\alpha}$ and $F_{\mu \rho} \partial_{\nu} A^{\rho}$, respectively, which are not gauge invariant. However, the first of these does not affect the supercharge (as may be verified by noting that the three-space integral of its zero component vanishes), and consequently it can be "improved" away. Likewise, the second becomes the gauge-invariant expression $F_{\mu \rho} F_{\nu}{ }^{\rho}$ when it is improved via the addition of the improvement term $-F_{\mu \rho} \partial^{\rho} A_{\nu}$. As required, this latter term is also conserved and has a zero-component with a vanishing three-integral. Thus, any failure of gauge invariance in this theory is at best only spurious.

This theory is clearly an example of the superconformal case in which the superconformal and $R_{5}$-symmetries are both unbroken. Indeed, each of these currents can be improved so that they together fill out the supercurrent supermultiplet [2]:

$$
J^{\mu} \equiv-\frac{1}{2} \bar{\sigma}^{\mu \dot{\alpha} \alpha} J_{\alpha \dot{\alpha}} \quad \text { where } \quad J_{\alpha \dot{\alpha}} \equiv 2 W_{\alpha} \bar{W}_{\dot{\alpha}} .
$$

Expanding this multiplet in components, we find

$$
\begin{aligned}
C_{\mu} \equiv & -\lambda \sigma_{\mu} \bar{\lambda} \\
\chi_{\mu \alpha} \equiv & -\left(\sigma_{\mu} \bar{\lambda}\right)_{\alpha} D-i\left(\sigma^{\nu} \bar{\lambda}\right)_{\alpha}\left(F_{\mu \nu}+\tilde{F}_{\mu \nu}\right) \\
\hat{T}_{\nu \mu} \equiv & \left(F_{\mu}{ }^{\rho}+\tilde{F}_{\mu}{ }^{\rho}\right)\left(F_{\rho \nu}-\tilde{F}_{\rho \nu}\right)+2 i D \tilde{F}_{\mu \nu} \\
& \quad+i\left(\partial_{\nu} \lambda\right) \sigma_{\mu} \bar{\lambda}-i \lambda \sigma_{\mu}\left(\partial_{\nu} \bar{\lambda}\right)+g_{\mu \nu} D^{2}
\end{aligned}
$$

Use of the relations in Eq. (3.11), as appropriate for a superconformal theory, then leads to the "improved" expressions for $j_{\mu}^{(5)}, j_{\mu}$, and $T_{\mu \nu}$ in this theory.

Given these results, let us now consider the same theory with an FI term. Indeed, this now becomes the simplest possible theory that can be constructed with an FI term:

$$
\begin{aligned}
\mathcal{L} & =\frac{1}{4}\left(\left.W^{\alpha} W_{\alpha}\right|_{\theta \theta}+\left.\bar{W}_{\dot{\alpha}} \bar{W}^{\dot{\alpha}}\right|_{\overline{\theta \theta}}\right)+\left.2 \xi V\right|_{\theta \theta \overline{\theta \theta}} \\
& =-\frac{1}{4} F^{2}-\frac{i}{2} \lambda \sigma^{\mu}\left(\partial_{\mu} \bar{\lambda}\right)+\frac{i}{2}\left(\partial_{\mu} \lambda\right) \sigma^{\mu} \bar{\lambda}+\frac{1}{2} D^{2}+\xi\left(D+\frac{1}{2} \square C\right)
\end{aligned}
$$

Because the $C$-term in Eq. (3.24) is a total derivative, it is legitimate to drop this term completely from any subsequent analysis. We then find that the equations of motion for this theory are unchanged from those of Eq. (3.19), except that we now

${ }^{\dagger}$ Note that the dropping of the $C$-term does not mean that we have passed to Wess-Zumino 
have $D=-\xi$. In other words, the equations of motion of this theory now take the form

$$
\bar{D}_{\dot{\alpha}} \bar{W}^{\dot{\alpha}}=2 \xi \text {. }
$$

Likewise, straightforwardly repeating the Noether procedure, it is easy to verify that the presence of the FI term induces the following additional $\xi$-dependent contributions to the Noether currents in Eq. (3.20):

$$
\begin{aligned}
\Delta j_{\mu}^{(5)} & =0 \\
\Delta j_{\mu \alpha} & =\xi\left(\sigma_{\mu} \bar{\lambda}\right)_{\alpha} \\
\Delta T_{\mu \nu} & =\xi g_{\mu \nu} D .
\end{aligned}
$$

There are several features to note concerning the results in Eq. (3.26). First, we see that the results for these Noether currents are completely gauge invariant. This is true for the full gauge invariance associated with the shifts in Eq. (2.3) as well as the restricted traditional $U(1)$ gauge symmetry which remains after truncation to Wess-Zumino gauge. This makes sense, since we have already seen from Table 1 that the FI term itself respects these symmetries as far as the action is concerned.

Second, we observe that this theory now effectively has a cosmological constant as a result of the FI term. Previously, without the FI term, equations of motion could be used to eliminate the final term $\frac{1}{2} g_{\mu \nu} D^{2}$ which appeared in $T_{\mu \nu}$. However, in the presence of an FI term, our equation of motion for $D$ becomes $D=-\xi$. As a result, this term now yields a constant $\frac{1}{2} \xi^{2} g_{\mu \nu}$. Combining this with the extra contribution $\xi g_{\mu \nu} D$ coming from Eq. (3.26) yields an overall constant $-\frac{1}{2} \xi^{2} g_{\mu \nu}$. This term is nothing but the effective cosmological constant $\Lambda=+\frac{1}{2} \xi^{2}$ induced by the FI term; indeed, $\Lambda>0$ (as required for such a broken-supersymmetry theory in flat space) because $g^{00}=-1$.

But most importantly for our purposes, we observe from Eq. (3.26) that the appearance of an FI term does not affect the $R_{5}$-current. This is entirely as expected: the FI term is composed of the $D$ and $C$ fields, and these are both entirely neutral under the chiral $R_{5}$-symmetry transformations. This, then, is an explicit Noetherderived verification of the fact that FI terms, in and of themselves, do not break $R_{5}$ symmetries. In particular, we have $\partial^{\mu} j_{\mu}^{(5)}=0$ both with and without the inclusion of the FI term. Thus, this property must remain true - even with the possible

gauge; in particular, we are continuing to operate in a completely general gauge. We have simply dropped the $C$-term because it is a total derivative and as such contains no physics at the classical level. Indeed, if we had retained the $C$-term in the following, we would have found that the corresponding equations of motion would have left $C$ entirely unconstrained; likewise, the corresponding Noether $R_{5}$-current and supercurrent would not have depended on $C$ in any way, and the Noether energy-momentum tensor would have accrued an extra $C$-dependent term $\frac{1}{2} \xi\left(g_{\mu \nu} \square-\partial_{\mu} \partial_{\nu}\right) C$ which is in the form of an improvement term - i.e., a term which is conserved (in this case both onand off-shell) and which makes no contribution to the corresponding physical Noether charge. It is therefore legitimate to drop the $C$-term from the Lagrangian, even in completely general gauge. 
addition of improvement terms - for any supercurrent supermultiplet which is to be associated with the FI term.

Having assembled at our disposal all the results we will need, we now seek to construct the corresponding supercurrent supermultiplet for this theory in the presence of an FI term. This should take the form

$$
J^{\mu} \equiv-\frac{1}{2} \bar{\sigma}^{\mu \dot{\alpha} \alpha} J_{\alpha \dot{\alpha}} \quad \text { where } \quad J_{\alpha \dot{\alpha}} \equiv 2 W_{\alpha} \bar{W}_{\dot{\alpha}}+\Xi_{\alpha \dot{\alpha}}
$$

where $\Xi_{\alpha \dot{\alpha}}$ represents the extra $\xi$-dependent contribution to the supercurrent supermultiplet. As discussed at the end of Sect. 3.3, $\Xi_{\alpha \dot{\alpha}}$ can be different depending on whether we work within the chiral or linear formalism. But what precisely is this contribution from the FI term in each case? That is the question to which we now turn.

\section{Analysis in the chiral formalism}

In this section, we address the issue of deriving $\Xi_{\alpha \dot{\alpha}}$ within the chiral formalism. We begin by reviewing the formalism of so-called "chiral compensators", as this will be our method for deriving the corresponding supercurrent. We then discuss the implications of these results for the existence of additional global symmetries in any theory with a non-zero FI term. Finally, we provide a proof that there does not exist any solution for $\Xi_{\alpha \dot{\alpha}}$ in which $R_{5}$-symmetry is conserved, and discuss one possible method by which such a proof might be evaded.

\subsection{Chiral compensator formalism: General outline}

Our chief interest in this paper concerns the manner in which a globally supersymmetric theory can be coupled to supergravity (i.e., be made locally supersymmetric). Of course, for a theory which exhibits a full superconformal invariance, the answer is simple: since the relevant currents are all conserved, they can be coupled directly to the fields of the the conformal supergravity multiplet $\left(b_{\mu}, \psi_{\mu \alpha}, e_{\mu}^{m}, a_{\mu}\right)$. By contrast, a supersymmetric theory which is not superconformal cannot be coupled directly to this multiplet. However, it is always possible to "promote" such a theory to a superconformal one by adding to the theory a set of additional fields called "conformal compensators" which artificially restore superconformal invariance to the theory. These conformal compensator fields are introduced in such a way that salient algebraic aspects of the original non-conformal theory can be reproduced from the conformal theory simply by assigning certain fixed values to these conformal compensators; indeed, assigning fixed values to such fields necessarily breaks any symmetries with respect to which these fields carry a charge. In this way, the entire anomaly structure of the original non-superconformal theory is embedded into the structure of the compensator fields. 
Given such conformal compensators, it is then straightforward to couple any globally super-Poincaré invariant theory to supergravity, regardless of its symmetry structure. We simply promote the theory to a superconformal theory by judiciously introducing appropriate conformal compensator fields, and then couple the resulting superconformal theory to the conformal gravity multiplet. As we shall see, setting the conformal compensator fields to fixed values then reproduces either our original theory or a theory whose symmetry properties are the same as those of our original theory.

As discussed in Sect. 3, many different constructions of this sort exist, each with its own set of conformal compensator fields. However, it can be shown [29] that only two such formulations may be regarded as minimal, meaning that the auxiliary-field formulation of supergravity to which the compensated theory may be coupled contains the minimal number of auxiliary degrees of freedom necessary to achieve equal numbers of bosonic and fermionic degrees of freedom. These are the chiral ("old minimal") and linear ("new minimal") formulations. In this section, we shall discuss the chiral compensator formalism, deferring a discussion of the linear compensator formalism to Sect. 5. In each case, we shall also emphasize how FI terms are incorporated into the formalism, as well as the consequences that result from doing so.

In general, the conformal (scaling) properties of a given quantity $X$ are determined by its so-called Weyl charge (or Weyl weight) $w_{X}$. Gauge vector superfields $V$ and gauge field-strength superfields $W_{\alpha}$ have Weyl weights $\left(w_{V}, w_{W_{\alpha}}\right)=(0,3 / 2)$, while constants (even dimensionful constants) have vanishing Weyl weights. The Weyl weights $w_{i}$ corresponding to chiral matter superfields $\Phi_{i}$ can vary depending on the theory in question. Since our superspace coordinates $(x, \theta)$ have Weyl weights $\left(w_{x}, w_{\theta}\right)=(-1,-1 / 2)$ respectively, we see that a globally supersymmetric theory will be conformally invariant only if its Kähler potential $K$ and superpotential $W$ have Weyl charges

$$
w_{K}=2, \quad w_{W}=3 .
$$

If the Kähler potential and superpotential of a given theory do not satisfy Eq. (4.1), then the theory is not conformal.

A similar situation exists for charges under $R_{5}$-transformations. Specifically, if the Kähler potential and superpotential of the theory do not have $R_{5}$-charges 0 and 2 respectively, then the theory also breaks $R_{5}$-symmetry. Note that while the $R_{5}$-charge of a given chiral superfield $\Phi_{i}$ is arbitrary, depending on the theory in question, a given left- (right-)chiral superfield $\Phi_{i}$ must have an $R_{5}$-charge $R_{i}^{(5)}$ and Weyl weight $w_{i}$ which satisfy

$$
R_{i}^{(5)}= \pm \frac{2}{3} w_{i}
$$

This condition ensures that a left- (right-)chiral superfield remains left- (right-)chiral under both $R_{5}$-rotations and Weyl rescalings [13]. By contrast, other types of 
multiplets satisfy different relations. For example, linear multiplets satisfy $R^{(5)}=$ $2(w-2) / 3$.

The relation in Eq. (4.2) is the minimum constraint needed for a general chiral superfield. In this context, we remark that all $R$-symmetries are essentially on the same footing in the absence of any coupling to supergravity; indeed, it is only the coupling to supergravity that selects one of these $R$-symmetries (here denoted $R_{5}$ ) to become local. Thus the question of which symmetry can be identified as $R_{5}$ is ultimately a formalism-dependent question. In the chiral formalism, $R_{5}$ corresponds to the charge assignments $R_{i}^{(5)}=2 / 3$, or $w_{i}=1$, for all chiral superfields $\Phi_{i}$. With this convention, $R_{5}$ may or may not actually be a symmetry of our original theory. By contrast, in Sect. 5, we shall see that there is no constraint on $R_{5}$ in the linear formalism; $R_{5}$ can be associated with whichever $R$-symmetry is preserved, and the use of the linear formalism presupposes that there is at least one such symmetry in our theory. However, it can be shown [7] that the linear formalism will not yield a supercurrent $J_{\alpha \dot{\alpha}}$ satisfying Eq. (3.7), and likewise will not yield a symmetric energymomentum tensor $T_{\mu \nu}$, unless all $R_{i}^{(5)}$ are equal.

Conformal (Weyl) symmetry and $R_{5}$-symmetry are closely related. By itself, $R_{5^{-}}$ symmetry is a $U(1)$ symmetry, but this is merely an ordinary $U(1)$ symmetry, not a full $U(1)$ superfield symmetry as in Eq. (2.4). However, when joined with conformal (Weyl) symmetry and so-called "special SUSY" transformations, $R_{5}$ fills out a full super-Weyl $U(1)_{\mathrm{SW}}$ superfield symmetry of the form in Eq. (2.4). In other words, $R_{5}$-symmetry is what remains of the full $U(1)_{\mathrm{SW}}$ superfield symmetry in its WessZumino gauge. The above relations between the $R_{5}$-charges and conformal $w$-weights for different kinds of superfields simply enforce the requirement that these superfields retain their defining characteristics (chiral, vector, linear, etc..) under $U(1)_{\mathrm{SW}}$. As a matter of normalization convention, a chiral superfield $\Phi_{i}$ will be defined to have charge $q_{\mathrm{SW}} \equiv R_{i}^{(5)}$ under $U(1)_{\mathrm{SW}}$.

If a given theory breaks conformal symmetry and/or $R_{5}$-symmetry, we can restore these symmetries by introducing two conformal compensator fields: a chiral superfield $\Sigma$, and its hermitian conjugate $\bar{\Sigma}$. These fields are respectively assigned $R_{5}$-charges $\pm 2 / 3$ and Weyl weights $w_{\Sigma}=w_{\bar{\Sigma}}=1$. Given such fields, our prescription for promoting our theory to a superconformal one is straightforward.

First, in the superpotential, we define the new fields $\widetilde{\Phi}_{i}$ through the relations

$$
\Phi_{i} \equiv\left(\frac{\Sigma}{\sqrt{3} M_{P}}\right) \widetilde{\Phi}_{i}
$$

where $M_{P}$ is the Planck mass and the factor of $\sqrt{3}$ is merely conventional. A similar definition holds for the conjugate fields $\Phi^{\dagger}$ and $\bar{\Sigma}$. Note that by construction, these new fields $\widetilde{\Phi}, \widetilde{\Phi}^{\dagger}$ have vanishing Weyl weights and $R_{5}$-charges. We then re-express our original fields $\Phi_{i}$ in terms of the new fields $\widetilde{\Phi}_{i}$. By contrast, any coupling $X_{n}$ that appears in the superpotential with mass dimension $n$ (such as a mass $m_{i j}$, or a 
Yukawa coupling $\left.y_{i j k}\right)$ is algebraically replaced with a corresponding superfield:

$$
X_{n} \rightarrow\left(\frac{\Sigma}{\sqrt{3} M_{P}}\right)^{n} \widetilde{X}_{n} .
$$

Here $\widetilde{X}_{n}$ can be viewed as another coupling with the same numerical value in fixed units. The net effect of these operations is thus to shift

$$
W \rightarrow \widehat{W} \equiv\left(\frac{\Sigma}{\sqrt{3} M_{P}}\right)^{3} \widetilde{W}
$$

where $\widetilde{W}$ has vanishing $R_{5}$-charge and Weyl weight. Thus, we have succeeded in constructing a new superconformal superpotential $\widehat{W}$.

Note that the shift from $\Phi_{i}$ to $\widetilde{\Phi}_{i}$ is merely an algebraic rewriting. By contrast, the replacement of the coupling $X_{n}$ with the superfield in Eq. (4.4) for $n \neq 0$ fundamentally changes the theory, producing a superconformal superpotential from a non-superconformal one. Of course, superpotentials $W$ without such couplings will already be superconformal. In such cases, we find that $\widehat{W}=W$.

In general, we also must modify our Kähler potential $K$. In this case, the procedure is easy. First, we may write $K=K\left(\Phi, \Phi^{\dagger}, V\right)=\sum_{n} K_{n}$ where $K_{n}$ has Weyl weight $n$ and vanishing $R_{5}$-charge. Given this form, we then define a new quantity

$$
\widetilde{K} \equiv K\left(\widetilde{\Phi}, \widetilde{\Phi}^{\dagger}, V\right)=\sum_{n}\left(\frac{\Sigma \bar{\Sigma}}{3 M_{P}^{2}}\right)^{-n / 2} K_{n}
$$

with vanishing Weyl weight and $R_{5}$-charge. In terms of $\widetilde{K}$, our new Kähler potential $\widehat{K}$ then takes the form

$$
\widehat{K} \equiv-\bar{\Sigma} \Sigma \exp \left(-\frac{\widetilde{K}}{3 M_{P}^{2}}\right)
$$

and once again we see that $\widehat{K}$ is guaranteed to be superconformal.

Given these definitions, we can now easily promote an arbitrary supersymmetric theory with broken superconformal invariance and broken $R_{5}$-symmetry to a theory in which both symmetries are restored: we simply replace

$$
K \rightarrow \widehat{K} \quad \text { and } \quad W \rightarrow \widehat{W} .
$$

Note that the kinetic terms for gauge fields are unaffected, since they are already superconformal. Now that we have a fully superconformal theory, we can "couple" this theory to conformal supergravity. Specifically, this means that we covariantize all superspace derivatives to make them local, and likewise replace our flat superspace integration measures with curved ones. This is equivalent to retaining our original superspace derivatives, but introducing an additional Lagrangian term which couples 
the flat-space supercurrent superfield $J_{\alpha \dot{\alpha}}$ to an appropriate corresponding supergravity multiplet (in this case, a superconformal one) [28]. As a final step, in order to return back to the symmetry structure and algebraic forms associated with our original theory, we simply set our compensator fields to fixed values,

$$
\Sigma, \bar{\Sigma} \rightarrow \sqrt{3} M_{P} .
$$

Indeed, since $\Sigma$ and $\bar{\Sigma}$ carry non-trivial $R_{5}$-charges and Weyl weights, setting these fields to have fixed values as in Eq. (4.9) has the net effect of breaking $R_{5}$-symmetry and superconformal invariance.

The result of this process is then a theory with the same symmetry structure as our original theory, but coupled to (Poincaré) supergravity. The quantity $M_{P}$, of course, describes the strength of this coupling, and we may compare the theory that results from this procedure with the original globally supersymmetric theory with which we started by taking the formal limit $M_{P} \rightarrow \infty$.

While it is clear that the replacements in Eq. (4.9) algebraically restore our original superpotential, reducing $\widehat{W} \rightarrow W$, it is perhaps less clear that making these replacements and taking the $M_{P} \rightarrow \infty$ limit algebraically restores our original Kähler term as well. However, we note that

$$
\begin{aligned}
\int d^{2} \theta d^{2} \bar{\theta} \widehat{K} & =\int d^{2} \theta d^{2} \bar{\theta}\left[-\bar{\Sigma} \Sigma \exp \left(-\frac{\widetilde{K}}{3 M_{P}^{2}}\right)\right] \\
& \rightarrow \int d^{2} \theta d^{2} \bar{\theta}\left[3 M_{P}^{2}+K+\mathcal{O}\left(M_{P}^{-4}\right)\right] \\
& =\int d^{2} \theta d^{2} \bar{\theta} K
\end{aligned}
$$

where we have used the fact that $\widetilde{K} \rightarrow K$ in passing to the second line. Thus, the Kähler portion of our original theory is algebraically restored as well. Note that in this discussion, we have not explicitly shown the additional supergravity terms which would ordinarily appear in the Lagrangian, as they are not relevant to the present discussion.

In this context, however, it is important to note that this "restoration" of the original theory is merely an algebraic illusion. In truth, our original theory has not been restored at all. Although our extra factors of $\left(\Sigma / \sqrt{3} M_{P}\right)$ and $\left(\bar{\Sigma} / \sqrt{3} M_{P}\right)$ have conveniently disappeared in this process, leaving behind what superficially looks like our original theory, we must recall that these factors also carried with them certain Weyl weights and $R_{5}$-charges. Thus, although the theory that emerges at the end of the day algebraically resembles our original theory, all of the non-trivial Weyl weights and $R_{5}$-charges have been stripped from the fields in question. Thus, the behavior of our final theory under conformal transformations and chiral $R_{5}$-rotations will be completely different than the behavior exhibited by our original theory.

This last point can be illustrated even more dramatically by considering a theory in which superconformal invariance is broken but $R_{5}$-symmetry is preserved. There 
is nothing that prevents us from using the above chiral compensator formalism in this case as well. However, if we use our chiral compensators to promote this theory to a fully superconformal theory and then attempt to return to our original theory following the above prescription, we find that our new theory has neither superconformal invariance nor $R_{5}$-symmetry. In other words, the $R_{5}$-symmetry of the original theory has been broken in the "hysteresis" process of promoting and then demoting the theory.

For this reason, it is critical that we continue to distinguish between our original theory, our promoted (compensated) theory, and the final theory that results after the compensator fields are "frozen" back to fixed values. Indeed, these are three independent theories with entirely different properties. Although the original theory and the final theory may algebraically resemble each other, they behave entirely differently under Weyl rescalings and chiral $R_{5}$-rotations. Thus, they ultimately cannot be identified with each other.

\subsection{Deriving the FI supercurrent supermultiplet in the chiral formalism}

We now turn to the question of deriving a supercurrent supermultiplet in the chiral formalism.

As discussed above, there are three distinct theories that come into play when discussing the chiral formalism: the original theory which lacks conformal symmetry (and which may or may not contain an unbroken $R_{5}$-symmetry), the compensated theory which exhibits a full superconformal invariance, and the final theory that results when our chiral compensator fields are "frozen" to fixed values. In principle, these are three distinct theories, and it is possible for each of them to have a different supermultiplet of currents. Indeed, there is also no guarantee that the currents associated with the (compensated) fully superconformal theory will, when subjected to the subsequent "freezing" process, reduce to the currents that might be calculated directly for the frozen theory. Thus, we must distinguish precisely which theory it is for which we seek to evaluate a supercurrent supermultiplet.

Clearly, all three theories will give rise to supercurrents and energy-momentum tensors which are conserved. This follows from the fact that all three theories exhibit unbroken supersymmetry and translational invariance. However, only the (compensated) fully superconformal theory will have a conserved $R_{5}$-current, for this is the only theory in which $R_{5}$-symmetry is guaranteed to be unbroken. Therefore, our procedure will be to calculate our supercurrent supermultiplet within the context of the fully superconformal compensated theory, and then to subject this current to the "freezing" process of setting our compensators to fixed values. We may then loosely identify this supercurrent as corresponding to our original uncompensated theory by taking the $M_{P} \rightarrow \infty$ limit.

Note that regardless of the $R_{5}$-symmetry properties of our original theory, this procedure is guaranteed to yield a supercurrent supermultiplet whose bottom com- 
ponent $j_{\mu}^{(5)}$ is not conserved. Moreover, we shall also find that this is generally not the same as calculating the currents directly in the final theory that emerges after "freezing". In other words, calculating our currents through the Noether procedure does not commute with the "freezing" process, and it matters whether our currents are calculated before or after the compensator fields are set to their fixed values.

Our interest in this paper concerns theories with FI terms, such as the pure $U(1)_{\mathrm{FI}}$ gauge theory in Eq. (3.24). This theory has $W=0$, and we may identify the Kähler potential of this theory as $K=\widetilde{K}=2 \xi V$, where $V$ is the $U(1)_{\mathrm{FI}}$ vector superfield. We can therefore use our chiral compensator formalism to promote the $\xi$-dependent part of the Lagrangian to the superconformal form

$$
\mathcal{L} \equiv \int d^{2} \theta d^{2} \bar{\theta}\left[-\bar{\Sigma} \Sigma \exp \left(-\frac{2 \xi V}{3 M_{P}^{2}}\right)\right] .
$$

We can then expand this expression in terms of the component fields of $V$, as in Eq. (2.1), and the component fields $\left\{\phi_{\Sigma}, \psi_{\Sigma}, F_{\Sigma}\right\}$ of $\Sigma$. Doing this to quadratic order in $\xi / M_{P}^{2}$, calculating the $R_{5}$-current in the resulting theory using the Noether procedure, and then setting $\Sigma$ and $\bar{\Sigma}$ to their fixed values $\sqrt{3} M_{P}$, we find the result

$$
j_{\mu}^{(5)}=-\frac{4}{3} \xi A_{\mu}-\frac{\xi^{2}}{18 M_{P}^{2}} \chi \sigma_{\mu} \bar{\chi}+\mathcal{O}\left(M_{P}^{-6}\right) .
$$

Note that this expression comes entirely from variations of the $\Sigma, \bar{\Sigma}$ chiral compensator fields, since all of the remaining matter fields have vanishing $R_{5}$-charges in the chiral formalism. Taking the $M_{P} \rightarrow \infty$ limit of Eq. (4.12) then yields the result

$$
j_{\mu}^{(5)}=-\frac{4}{3} \xi A_{\mu},
$$

and this may be identified as the bottom component of the superfield

$$
\Xi_{\alpha \dot{\alpha}}^{(C)}=\frac{2 \xi}{3}\left[D_{\alpha}, \bar{D}_{\dot{\alpha}}\right] V .
$$

This, then, is the result for the FI contribution $\Xi_{\alpha \dot{\alpha}}^{(C)}$ to the supercurrent superfield in the chiral formalism, whereupon we conclude that the total supercurrent supermultiplet for the theory in Eq. (3.24) is given by

$$
J_{\alpha \dot{\alpha}}^{(C)}=2 W_{\alpha} \bar{W}_{\dot{\alpha}}+\frac{2 \xi}{3}\left[D_{\alpha}, \bar{D}_{\dot{\alpha}}\right] V
$$

in the chiral formalism. Indeed, this is precisely the result quoted in Ref. [25].

It is straightforward to generalize this result to a sigma model with arbitrary Kähler potential $K$ and arbitrary superpotential $W$. The corresponding supercurrent supermultiplet in the chiral formalism is then given by the general expression

$$
J_{\alpha \dot{\alpha}}^{(C)}=-g^{i \overline{1}}\left(D_{\alpha} \Phi_{i}\right)\left(\bar{D}_{\dot{\alpha}} \bar{\Phi}_{\overline{1}}\right)+\frac{1}{3}\left[D_{\alpha}, \bar{D}_{\dot{\alpha}}\right] K,
$$


where the Kähler metric is given by

$$
g^{i \overline{1}} \equiv \frac{\partial^{2} K}{\partial \Phi_{i} \partial \bar{\Phi}_{\overline{1}}} .
$$

Indeed, the supercurrent in Eq. (4.16) is independent of the superpotential $W$ except through the equations of motion.

It is important to note that we derived these results by evaluating our Noether currents in the compensated superconformal theory prior to "freezing" our compensators to fixed values and taking the $M_{P} \rightarrow \infty$ limit. By contrast, if we had analyzed the freezing properties of the Lagrangian in Eq. (4.11) directly, we would have found that only one term ultimately survives:

$$
\mathcal{L}=\frac{\xi}{3 M_{P}^{2}} \phi_{\Sigma} \bar{\phi}_{\Sigma}\left(D+\frac{1}{2} \square C\right)+\ldots
$$

where $\phi_{\Sigma}$ and $\bar{\phi}_{\Sigma}$ are respectively the lowest (scalar) components of our compensator fields $\Sigma$ and $\bar{\Sigma}$. Of course, with the substitutions $\Sigma, \bar{\Sigma} \rightarrow \sqrt{3} M_{P}$, we recognize Eq. (4.18) as our original FI Lagrangian. However, since the $D$ - and $C$-fields have vanishing $R_{5}$-charges, this term does not make any contribution to $j_{\mu}^{(5)}$. This is therefore an explicit demonstration that the process of calculating a Noether current does not commute with the process of freezing the compensators to fixed values and taking the $M_{P} \rightarrow \infty$ limit.

Once again, we stress that the results in Eqs. (4.14) and (4.15) correspond to the conformally compensated theory whose Kähler contributions to the Lagrangian are given in Eq. (4.11). In particular, our expression for $j_{\mu}^{(5)}$ in Eqs. (4.12) and (4.13) is nothing but the result of applying the "freezing" process to the Noether current associated with Eq. (4.11). However, as with any such results derived through the chiral formalism, these currents do not correspond to the original theory in Eq. (3.24) with which we started. Indeed, our original theory in Eq. (3.24) has two manifest symmetries which are crucial and which are preserved in spite of the appearance of a non-zero FI term: $R_{5}$-invariance and $U(1)_{\mathrm{FI}}$ invariance. Both of these symmetries are explicitly broken in the results of Eqs. (4.14) and (4.15). In other words, there is no way in which we can connect the bottom component of the superfield in Eq. (4.15) to the $j_{\mu}^{(5)}$ Noether current derived in Sect. 3.4, with or without the addition of any possible improvement terms. Thus, we see that the $U(1)_{\text {FI }}$ gauge non-invariance of the result in Eq. (4.15) is an artifact of the chiral compensator formalism, and is not a property of the underlying physics of our original theory. In other words, in a Noether sense, no supercurrent supermultiplet exists for a theory with a non-zero FI term in the chiral formalism, independent of the compensators.

Despite this fact, the procedure we have followed does describe one of the "minimal" methods by which a theory such as that in Eq. (3.24) might be coupled to supergravity. Thus, the broken $U(1)_{\text {FI }}$ and $R_{5}$-symmetries of the supercurrent will indeed be of relevance insofar as this coupling to supergravity is concerned, with far-reaching consequences that we shall now explore. 


\subsection{The symmetry structure of theories with non-zero FI terms in the chiral formalism}

In this section, we shall explore the structure of local and global symmetries that appear in theories with non-zero FI terms in the chiral formalism. We shall begin by describing the general symmetry structure of such theories and the way in which it emerges. We shall then provide an explicit example.

\subsubsection{General symmetry structure}

In order for the chiral compensator formalism to be sensible, our Lagrangian must at all times exhibit manifest invariance under appropriate gauge transformations. However, the gauge transformations in question depend on the theory in which one is working.

In our original theory prior to the introduction of chiral compensators, we expect to have an unbroken local $U(1)$ gauge symmetry associated with our FI term. For reasons to become clear shortly, we shall refer to this symmetry as $U(1)_{\mathrm{FI}}^{\prime}$. Note that in the presence of a non-zero FI term, the Kähler potential will no longer be $U(1)_{\mathrm{FI}}^{\prime}$ gauge invariant: under a $U(1)_{\mathrm{FI}}^{\prime}$ gauge transformation

$$
V \rightarrow V+i\left(\Lambda-\Lambda^{\dagger}\right)
$$

the Kähler potential transforms as

$$
K \rightarrow K+2 i \xi\left(\Lambda-\Lambda^{\dagger}\right)
$$

Another way of saying this is that gauge transformations induce Kähler transformations, a fact first noticed in Ref. [11] and recently emphasized in Ref. [25]. However, this does not disturb the $U(1)_{\mathrm{FI}}^{\prime}$ gauge invariance of the theory, since the corresponding $D$-field within $V$ is gauge invariant.

This situation changes dramatically upon introduction of the chiral compensators. Now denoting our FI gauge-transformation group as $U(1)_{\mathrm{FI}}$ in the superconformal chirally-compensated theory, we once again see that gauge transformations of the form in Eq. (4.19) induce transformations of the Kähler potential of the form in Eq. (4.20). Note that this is true for both $K$ and $\widetilde{K}$, since the relevant term $2 \xi V$ within $K$ does not experience a rescaling under Eq. (4.6) when passing to $\widetilde{K}$. However, in the superconformal chirally-compensated theory, our final Kähler potential $\widehat{K}$ (or more precisely, its corresponding $D$-term) must be neutral under $U(1)_{\mathrm{FI}}$ symmetries. This in turn then forces us to assign a $U(1)_{\mathrm{FI}}$ charge to our chiral compensator fields, i.e.,

$$
Q_{\Sigma, \bar{\Sigma}}= \pm \frac{2 \xi}{3 M_{P}^{2}}
$$

The fact that the chiral compensator fields carry $U(1)_{\mathrm{FI}}$ charges in the presence of an FI term has three immediate consequences. 
First, this implies that the $U(1)_{\text {FI }}$ gauge symmetry — just like the Weyl symmetry and the $R_{5}$-symmetry which together form the super-Weyl $U(1)_{\mathrm{SW}}$ symmetry — will be broken when the compensator fields are set to fixed values. This explains the lack of $U(1)_{\mathrm{FI}}$ gauge invariance exhibited by the corresponding supercurrent supermultiplet after the compensator fields are "frozen". Moreover, after our chirally-compensated superconformal theory is coupled to supergravity, the $U(1)_{\mathrm{SW}}$-symmetry becomes local, leading to a $U(1)_{\text {SW }}$ gauge symmetry in addition to the $U(1)_{\text {FI }}$ gauge symmetry. The first symmetry is associated with the gauge boson $b_{\mu}$ which appears within the supergravity multiplet and which corresponds directly to $R_{5}$-transformations, while the second symmetry is associated with the $A_{\mu}$ component field within $V$. Because the $\Sigma$ field simultaneously carries both a non-zero $U(1)_{\mathrm{SW}}$-charge $q_{\mathrm{SW}}=2 / 3$ and a non-zero $U(1)_{\mathrm{FI}}$-charge given in Eq. (4.21), setting this field to a fixed value implies that our local gauge symmetry $U(1)_{\mathrm{SW}} \times U(1)_{\mathrm{FI}}$ will be broken down to a single axial $U(1)_{A}$ subgroup [12]:

$$
U(1)_{\mathrm{SW}} \times U(1)_{\mathrm{FI}} \rightarrow U(1)_{A} \equiv U(1)_{\mathrm{FI}}-\frac{\xi}{M_{P}^{2}} U(1)_{\mathrm{SW}}
$$

This residual $U(1)_{A}$ symmetry persists in the effective theory at energy scales below $M_{P}$, with a corresponding gauge boson

$$
A_{\mu}^{\prime} \equiv \frac{1}{\sqrt{1+\xi^{2} / M_{P}^{4}}}\left(A_{\mu}-\frac{\xi}{M_{P}^{2}} b_{\mu}\right) \text {. }
$$

For $\xi \ll M_{P}^{2}$, we see that this symmetry is mostly $U(1)_{\mathrm{FI}}$ itself.

However, there is also a second important consequence of the fact that our chiral compensator fields $(\Sigma, \bar{\Sigma})$ carry a $U(1)_{\mathrm{FI}}$ charge. Since our superpotential $W$ must be $U(1)_{\mathrm{FI}}$ neutral, we see that $\widetilde{W}$ must carry a $U(1)_{\mathrm{FI}}$ charge

$$
Q_{\widetilde{W}}=-\frac{2 \xi}{M_{P}^{2}}
$$

This places important restrictions on the superpotential structure of our theory. In general, $\widetilde{W}$ may contain trilinear terms of the form $y_{i j k} \widetilde{\Phi}_{i} \widetilde{\Phi}_{j} \widetilde{\Phi}_{k}$, mass terms of the form $m_{i j} \widetilde{\Phi}_{i} \widetilde{\Phi}_{j}$, and so forth. However, if it is possible to assign $U(1)_{\mathrm{FI}}$ charges to all of the $\widetilde{\Phi}$ fields such that each of the terms in $\widetilde{W}$ transforms with a uniform charge under $U(1)_{\mathrm{FI}}$ transformations, then this tells us something additional about the original theory that we had prior to introducing the compensators. In particular, this tells us that our original theory must exhibit an invariance under not only the local $U(1)_{\mathrm{FI}}^{\prime}$ symmetry, but also under an additional global $R$-symmetry, to be denoted $R^{\prime}$. If our original symmetry had an $R_{5}$-invariance as well, then this additional global $R^{\prime}$ symmetry may or may not coincide with $R_{5}$. This ultimately depends on the structure of the theory. 
Finally, in a similar vein, there are also additional symmetry repercussions for the chirally-compensated theory. Just as the local $U(1)_{\mathrm{FI}}$ invariance of the chirallycompensated theory implies the existence of an additional global symmetry $R^{\prime}$ in the original uncompensated theory, a similar thing happens in reverse: the local $U(1)_{\mathrm{FI}}^{\prime}$ symmetry of the original uncompensated theory implies the existence of an additional global symmetry in the chirally-compensated theory. To see why this is the case, let us recall that our original uncompensated theory had a local $U(1)_{\mathrm{FI}}^{\prime}$ invariance under which our matter fields $\widetilde{\Phi}_{i}$ were assigned charges leading to an invariant superpotential $W(\Phi)$. We may therefore promote this symmetry into the chirally-compensated theory by choosing our chiral compensator fields $\Sigma, \bar{\Sigma}$ to be neutral under this $U(1)_{\text {FI }}^{\prime}$ symmetry. Of course, in the chirally-compensated theory, this choice can only make sense if this new $U(1)_{\mathrm{FI}}^{\prime}$ symmetry is no longer associated with shifts in the gauge field supermultiplet $V$ or in the Kähler potential $\widehat{K}-i . e$., if this symmetry is no longer local, but global. Indeed, in the chirally-compensated theory, it is not $U(1)_{\mathrm{FI}}^{\prime}$ but $U(1)_{\mathrm{FI}}$ which is associated with the gauge shifts in Eq. (4.19). Nevertheless, in the chirally-compensated theory, we see that our original $U(1)_{\mathrm{FI}}^{\prime}$ symmetry leaves behind a global remnant which is disconnected from $U(1)_{\text {FI }}$ gauge transformations. Indeed, this is a bona-fide global symmetry of the chirally-compensated theory, with

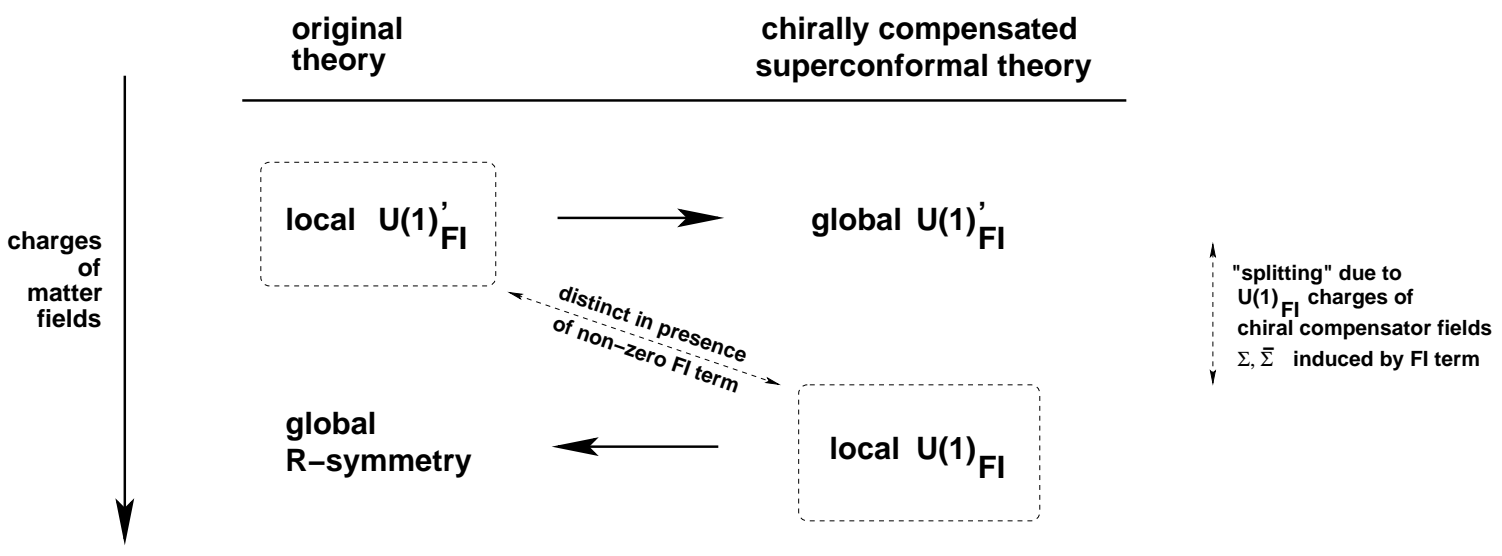

Figure 2: Comparison between the symmetries of our original theory and those of our chirally-compensated superconformal theory. In general, the existence of a local $U(1)_{\mathrm{FI}}$ symmetry in either theory implies the existence of a corresponding global symmetry in the other theory. In the presence of a non-vanishing FI term, the local $U(1)_{\text {FI }}$ symmetries in the two theories are distinct as a result of the non-vanishing $\xi$-dependent $U(1)_{\mathrm{FI}}$-charges of the chiral compensator fields $\Sigma, \bar{\Sigma}$. Thus, in the chiral formalism, the presence of a non-zero FI term requires the existence of additional global symmetries in both theories. However, when the FI term is eliminated, the local $U(1)_{\mathrm{FI}}$ symmetries in both theories can be identified with each other. In this case, the global $U(1)_{\mathrm{FI}}$ symmetry within the local $U(1)_{\text {FI }}$ symmetry of either theory serves as the global symmetry required by the local $U(1)_{\mathrm{FI}}$ symmetry of the other theory, and no additional global symmetries are needed in either theory. 
charge assignments for the matter fields $\widetilde{\Phi}_{i}$ and chiral compensator fields $\Sigma, \bar{\Sigma}$ which are distinct from those corresponding to the gauge symmetry $U(1)_{\mathrm{FI}}$. Under the subsequent "freezing" process in which $\Sigma, \bar{\Sigma}$ are set to fixed values, we have seen that our local $U(1)_{\mathrm{FI}}$ is ultimately broken. However, our global $U(1)_{\mathrm{FI}}^{\prime}$ symmetry remains intact.

We emphasize that this entire structure, with matching local and global symmetries for both the original theory and the chirally-compensated theory, only arises in the presence of a non-zero FI term. Without a non-zero FI term, our Kähler potentials $K$ and $\widehat{K}$ would both be invariant under $U(1)_{\mathrm{FI}}$ transformations. Thus the $U(1)_{\mathrm{FI}}$ and $U(1)_{\mathrm{FI}}^{\prime}$ symmetries would coincide between our original and chirallycompensated theories, and neither would trigger the existence of a global symmetry in the opposite theory. Indeed, it is only because the non-zero FI term requires the introduction of chiral compensator fields $\Sigma, \bar{\Sigma}$ with non-zero $U(1)_{\text {FI }}$ charges that these two symmetries become distinct, with each implying the existence of an additional global symmetry. This situation is illustrated in Fig. 2,

Needless to say, the fact that non-zero FI terms require the existence of additional global symmetries has far-reaching consequences. Indeed, it is currently believed (and indeed proven in the context of string theory [30, 31, 32]) that such additional global symmetries are inconsistent for any theory which is ultimately coupled to gravity. Thus, assuming this "folk" theorem to be true for all classes of gravitycoupled theories, we then conclude that theories with non-zero FI terms cannot be consistently coupled to supergravity in the chiral formalism.

\subsubsection{An explicit example}

For clarity, we shall now illustrate these ideas with a concrete example. Let us consider a simple $R_{5}$-invariant toy model which involves a single $U(1)_{\text {FI }}^{\prime}$ gauge group with a non-trivial FI term, and matter content comprising three chiral superfields $\Phi_{i}, i=1,2,3$. We shall imagine that these chiral superfields carry charges under $U(1)_{\mathrm{FI}}^{\prime}$, as well as under a global $R_{5}$-symmetry, in the manner indicated in Table 3 . We have also shown the charges for the $U(1)_{\mathrm{FI}}^{\prime}$ gaugino field $\lambda_{\alpha}$. Given these charge assignments, the most general renormalizable superpotential invariant under these symmetries is given by

$$
W=y_{1} \Phi_{1} \Phi_{2} \Phi_{3}+y_{2} \Phi_{3}^{3} .
$$

We will also assume that the matter field contribution $K^{\prime}$ to the Kähler potential takes the minimal form

$$
K^{\prime}=\Phi_{1} e^{-V} \Phi_{1}^{\dagger}+\Phi_{2} e^{V} \Phi_{2}^{\dagger}+\Phi_{3} \Phi_{3}^{\dagger}
$$

and that the full Kähler potential is given by $K=K^{\prime}+2 \xi V$, where $V$ denotes the vector superfield associated with $U(1)_{\mathrm{FI}}^{\prime}$. Note that this toy theory is completely $R_{5}$-invariant, with dilatation invariance spoiled only by the presence of the FI term. 


\begin{tabular}{||c||c|c||}
\hline \hline Field & $U(1)_{\mathrm{FI}}^{\prime}$ & $R_{5}$ \\
\hline \hline$\Phi_{1}$ & +1 & $2 / 3$ \\
$\Phi_{2}$ & -1 & $2 / 3$ \\
$\Phi_{3}$ & 0 & $2 / 3$ \\
\hline$\lambda_{\alpha}$ & 0 & 1 \\
\hline \hline
\end{tabular}

Table 3: $U(1)_{\mathrm{FI}^{-}}^{\prime}$ and $R_{5}$-charges of the fields in our toy model, as discussed in the text.

This model therefore contains only two symmetries: $U(1)_{\mathrm{FI}}^{\prime}$ and $R_{5}$, the first local and the second global.

When this theory is promoted to a superconformal theory in the chiral formalism through the introduction of the chiral compensators $\Sigma$ and $\bar{\Sigma}$, several changes occur. First, the matter fields of the theory are rescaled according to Eq. (4.3). Since the chiral compensators are forced to carry $U(1)_{\text {FI }}$ charge according to Eq. (4.21), we see that the resulting charges of the rescaled matter fields $\widetilde{\Phi}_{i}$ are shifted relative to their original $U(1)_{\mathrm{FI}}^{\prime}$ charges. In this way we determine that $U(1)_{\mathrm{FI}}$ and $U(1)_{\mathrm{FI}}^{\prime}$ are now distinct symmetries. Likewise, the shift in the Kähler potential from $K$ to $\widehat{K}$ defined in Eq. (4.7) restores conformal (Weyl) symmetry to the theory, even in the presence of an FI term, thereby generating a full $U(1)_{\text {SW }}$ super-Weyl symmetry as well. Table 4 lists the charges of the matter fields $\Phi_{i}$, the compensator field $\Sigma$, the $U(1)_{\mathrm{FI}}$ gaugino field $\lambda_{\alpha}$, and the gravitino field $\psi_{\mu \alpha}$ under these three $U(1)$ symmetries, along with their $R_{5}$-charges and $w$-weights.

Note that this theory contains two distinct FI-related symmetries, $U(1)_{\mathrm{FI}}$ and $U(1)_{\mathrm{FI}}^{\prime}$, yet only one FI vector superfield $V$. In the fully superconformal compensated

\begin{tabular}{||c||c|c|ccc||}
\hline \hline Field & $U(1)_{\mathrm{FI}}^{\prime}$ & $U(1)_{\mathrm{FI}}$ & $U(1)_{\mathrm{SW}}:$ & $R_{5}$ & Weyl \\
\hline \hline$\widetilde{\Phi}_{1}$ & +1 & $1-2 \xi / 3 M_{P}^{2}$ & 0 & 0 & 0 \\
$\widetilde{\Phi}_{2}$ & -1 & $-1-2 \xi / 3 M_{P}^{2}$ & 0 & 0 & 0 \\
$\widetilde{\Phi}_{3}$ & 0 & $-2 \xi / 3 M_{P}^{2}$ & 0 & 0 & 0 \\
\hline$\Sigma$ & 0 & $2 \xi / 3 M_{P}^{2}$ & $2 / 3$ & $2 / 3$ & 1 \\
\hline$\lambda_{\alpha}$ & 0 & 0 & $*$ & 1 & $3 / 2$ \\
$\psi_{\mu \alpha}$ & 0 & 0 & $*$ & 1 & $3 / 2$ \\
\hline \hline
\end{tabular}

Table 4: The symmetry structure of the chirally-compensated superconformal version of the toy model originally defined in Table 3 . We list the unbroken symmetries that exist in this model, along with the corresponding charges of the matter fields $\widetilde{\Phi}_{i}$, the chiral compensator field $\Sigma$, the $U(1)_{\mathrm{FI}}$ gaugino $\lambda_{\alpha}$, and the gravitino $\psi_{\mu \alpha}$. An entry ' $*$ ' indicates that the corresponding field is not a chiral superfield, and therefore its $R_{5}$ - and Weyl-charges cannot be packaged as a chiral charge under $U(1)_{\mathrm{SW}}$. 
theory, $V$ is associated with $U(1)_{\mathrm{FI}}$. As a result, $U(1)_{\mathrm{FI}}$ is a fully local supersymmetric $U(1)$ gauge symmetry in this theory. By contrast, because $U(1)_{\mathrm{FI}}^{\prime}$ lacks its own vector gauge superfield, we see that the $U(1)_{\mathrm{FI}}^{\prime}$ symmetry is neither local nor fully supersymmetric; rather, it is global, and it is only a Wess-Zumino remnant corresponding to an ordinary global $U(1)$ symmetry. Thus, borrowing the terminology introduced below Eq. (2.4), we see that $U(1)_{\mathrm{FI}}$ is both local and "big", while $U(1)_{\mathrm{FI}}^{\prime}$ is global and "little".

The full symmetry content of the chirally-compensated superconformal theory thus consists of three $U(1)$ symmetries: $U(1)_{\mathrm{FI}}^{\prime}, U(1)_{\mathrm{FI}}$, and $U(1)_{\mathrm{SW}}$. Note that this last symmetry is an $R$-type symmetry - i.e., a symmetry under which the superspace $\theta$-coordinate is charged, or equivalently a symmetry under which the superpotential $\widehat{W}$ is charged. By contrast, the two FI symmetries are not $R$-type. However, writing our three $U(1)$ symmetries in this way is only a basis choice, and we can express the symmetries of this model in terms of any linear combinations of these symmetries that we wish. One particularly important linear combination that we may define is

$$
R_{G} \equiv\left(\frac{\xi}{M_{P}^{2}}\right)^{-1}\left[U(1)_{\mathrm{FI}}^{\prime}-U(1)_{\mathrm{FI}}\right]+U(1)_{\mathrm{SW}}
$$

As a result of its definition, we see that $R_{G}$ is a global, "little", $R$-type symmetry. Under this symmetry the matter fields $\widetilde{\Phi}_{i}$ each have $R_{G}$-charge $2 / 3$, while the compensator fields $\Sigma, \bar{\Sigma}$ are uncharged. However, we emphasize that $R_{G}$ is not an additional symmetry of the theory, but merely a recasting of our global $U(1)_{\mathrm{FI}}^{\prime}$ symmetry into a new basis. Indeed, in all bases, our chirally-compensated superconformal theory contains only one independent global symmetry.

Let us now examine how the symmetry properties of this theory are altered by the freezing of the compensators. Since $\Sigma$ and $\bar{\Sigma}$ transform non-trivially under both $U(1)_{\mathrm{FI}}$ and $U(1)_{\mathrm{SW}}$, these symmetries will not be preserved individually in the frozen theory. However, as discussed above, a linear combination of these two symmetries, namely the gauged $R$-symmetry $U(1)_{A}$ of Eq. (4.22), is preserved in this theory. In addition, there is also a global $U(1)$ symmetry which survives: this may be alternatively interpreted as $U(1)_{\mathrm{FI}}^{\prime}$, as in Table 5, or as the additional $R_{G}$ symmetry defined in Eq. (4.27). Thus, after the compensator fields are frozen, the symmetry structure of our theory reduces to that shown in Table 5 .

As we explicitly see from this example, the symmetry structure that survives after the chiral compensators are frozen contains not only a single $U(1)_{A}$ gauge symmetry, but also an exact global symmetry $U(1)_{\mathrm{FI}}^{\prime}$. Indeed, this is the entire symmetry structure that survives, even in cases such as this in which the original theory is $R_{5}$-symmetric. Moreover, we observe that this structure remains intact for all $\xi \neq 0$. However, for $\xi=0$, we observe that $U(1)_{\mathrm{FI}}^{\prime}$ and $U(1)_{A}$ become redundant. In this case, the local $U(1)_{A}=U(1)_{\mathrm{FI}}^{\prime}$ symmetry survives, but no additional independent global symmetry remains in the theory. 


\begin{tabular}{||c||c|c||}
\hline \hline Field & $U(1)_{\mathrm{FI}}^{\prime}$ & $U(1)_{A}$ \\
\hline \hline$\widetilde{\Phi}_{1}$ & +1 & $1-2 \xi / 3 M_{P}^{2}$ \\
$\widetilde{\Phi}_{2}$ & -1 & $-1-2 \xi / 3 M_{P}^{2}$ \\
$\widetilde{\Phi}_{3}$ & 0 & $-2 \xi / 3 M_{P}^{2}$ \\
\hline$\lambda_{\alpha}$ & 0 & $-\xi / 3 M_{P}^{2}$ \\
$\psi_{\mu \alpha}$ & 0 & $-\xi / 3 M_{P}^{2}$ \\
\hline \hline
\end{tabular}

Table 5: The symmetry structure of the final version of our toy model in Table 4, after the compensator fields are "frozen" to their fixed values in the chiral formalism. We list the charges of the matter fields $\widetilde{\Phi}_{i}$, the $U(1)_{\mathrm{FI}}$ gaugino $\lambda_{\alpha}$, and the gravitino $\psi_{\mu \alpha}$. Note that the $R_{5}$-symmetry of our original model in Table 3 is broken; likewise, the local $U(1)_{\mathrm{FI}}^{\prime}$ gauge symmetry of our original model has also been broken, leaving behind only a global remnant, while the $U(1)_{\mathrm{FI}}$ gauge symmetry of the superconformal version of our theory has been broken entirely.

\subsection{Proof of the non-existence of an FI supercurrent supermultiplet with conserved $R_{5}$-symmetry in the chiral formalism}

As we have discussed, the supercurrent supermultiplet in the chiral formalism must always satisfy the constraint in Eq. (3.6), where $S$ is a chiral supermultiplet. However, even though the chiral formalism can accommodate the special case in which $R_{5}$-symmetry is preserved (such as for FI terms), this feature is not enforced by the formalism itself. Indeed, in the chiral formalism, the bottom component of the supercurrent supermultiplet is not conserved.

This result makes sense, since the supercurrent supermultiplet in this formalism actually corresponds not to our original global theory (in which $R_{5}$-symmetry is preserved), but to the chirally-compensated version of this theory (in which $R_{5}$-symmetry is broken). However, strictly speaking, this feature prevents us from associating the resulting supercurrent supermultiplet with our original global theory. Indeed, no possible improvement term can allow us to connect the resulting value of $C_{\mu}$ with an improved Noether current $j_{\mu}^{(5)}$. A natural question, therefore, is whether it might be possible to construct a supercurrent supermultiplet in the chiral formalism which does exhibit $R_{5}$-symmetry conservation, perhaps as a special case.

We shall now show that this cannot be done. Specifically, we shall assume the constraint in Eq. (3.6), and then attempt to impose $R_{5}$-symmetry conservation by hand as an additional constraint. By imposing both constraints simultaneously, we shall derive a condition on the most general supercurrent supermultiplet $J_{\alpha \dot{\alpha}}^{(C)}$ in the chiral formalism that can be consistent with $R_{5}$-symmetry conservation, i.e., consistent with the requirement that $\partial^{\mu} j_{\mu}^{(5)}=0$.

Since $j_{\mu}^{(5)}$ is by definition the lowest component of the supercurrent supermultiplet 
$J_{\mu}$, the $R_{5}$-symmetry constraint can be expressed as the superfield constraint

$$
\partial^{\mu} J_{\mu}=-\frac{i}{4}\left\{D^{\alpha}, \bar{D}^{\dot{\alpha}}\right\} J_{\alpha \dot{\alpha}}=0,
$$

where $J_{\alpha \dot{\alpha}}$ is defined in Eq. (3.5). However, we now use the chiral-case constraint in Eq. (3.6), along with the fact that an arbitrary chiral superfield $S$ can be written, without loss of generality, in terms of an unconstrained vector superfield $T_{S}$ as

$$
S=\bar{D}^{2} T_{S} .
$$

This enables us to write

$$
\left\{D^{\alpha}, \bar{D}^{\dot{\alpha}}\right\} J_{\alpha \dot{\alpha}}=D^{2} \bar{D}^{2} T_{S}-\bar{D}^{2} D^{2} \bar{T}_{S},
$$

whereupon we find that any supercurrent supermultiplet $J_{\alpha \dot{\alpha}}$ in the chiral formalism will be consistent with unbroken $R_{5}$-symmetry if and only if

$$
D^{2} \bar{D}^{2} T_{S}=\bar{D}^{2} D^{2} \bar{T}_{S} .
$$

Thus, in cases where superconformal invariance is broken while $R_{5}$-symmetry is preserved, Eq. (4.31) should follow as a direct consequence of the equations of motion of the theory. Note that in cases for which $T_{S}$ turns out to be real, Eq. (4.31) reduces to

$$
\left[D^{2}, \bar{D}^{2}\right] T_{S}=0
$$

or equivalently

$$
\square T_{S}=-\frac{i}{2}\left(D \sigma^{\mu} \bar{D}\right) \partial_{\mu} T_{S} .
$$

So what goes wrong in the case of an FI term? Given the above results, the answer is quite easy to see.

First, we observe that in the case of an FI term, the corresponding contribution $\Xi_{\alpha \dot{\alpha}}^{(C)}$ to the total supercurrent supermultiplet would have to correspond to a case in which $T_{S}$ is proportional to the real vector superfield $V$ itself (up to the addition of harmless terms annihilated by $D^{2} \bar{D}^{2}$ ). This result follows immediately from dimensional analysis and Lorentz symmetry. Moreover, as we have already seen in Sect. 4.2, this result is also natural from the supposition that the FI term should follow the expectations associated with a general sigma model, leading to the result in Eq. (4.15). Indeed, given the result in Eq. (4.15), we find that $S=-(\xi / 3) \bar{D}^{2} V$, whereupon we have

$$
T_{S}=-\frac{\xi}{3} V .
$$

Thus, once again, we find that $T_{S}$ would be proportional to $V$.

Unfortunately, the problem with having $T_{S}$ proportional to $V$ is that the constraint equation (4.33) on $T_{S}$ does not hold as a result of the equations of motion 
for $V$. Instead, Eq. (4.33) now has the devastating effect of imposing a structural truncation directly on the multiplet $V$ which goes beyond its equations of motion. Specifically, with $V$ expanded as in Eq. (2.1) and with $T_{S} \sim V$, we find that Eq. (4.33) becomes

$$
\begin{aligned}
\partial_{\mu} A^{\mu} & =0 \\
\partial_{\mu} D & =-\partial_{\mu} \square C \\
\square M & =\square N=0 \\
\square \chi_{\alpha} & =-i\left(\sigma^{\mu} \partial_{\mu} \bar{\lambda}\right)_{\alpha} .
\end{aligned}
$$

These constraints go far beyond the equations of motion for $V$ : they imply that $V$ must be a linear multiplet, up to possible Kähler transformations which do not change the physics. However, this represents a severe and unjustified truncation of the unconstrained real vector multiplet $V$ with which we started.

Thus, we reach a contradiction: if we wish to impose $R_{5}$-conservation on $\Xi_{\alpha \dot{\alpha}}^{(C)}$ in the chiral formalism, we find that $V$ cannot be the unconstrained real gauge field with which we started, and with which we constructed our FI term. Instead, we see that $V$ must actually be another beast entirely — a linear multiplet, up to Kähler transformations - if Eq. (4.15) is to remain valid for this theory. But if $V$ is presumed constrained according to Eq. (4.35), then our supposed FI term with which we began was not an FI term at all, but something entirely different. Thus, we conclude that we cannot self-consistently impose $R_{5}$-conservation on the supercurrent supermultiplet for the FI term in the chiral formalism.

This, then, is the fundamental impasse that emerges upon attempting to construct an FI supercurrent supermultiplet in the chiral formalism while simultaneously demanding manifest $R_{5}$-invariance. Dimensional analysis indicates that any supercurrent that could possibly correspond to the FI term in the chiral formalism must have $T_{S} \sim V$. However, self-consistency then requires that the multiplet $V$ be truncated in a way that transcends its general equations of motion. This in turn prohibits from $V$ from corresponding to the real gauge field with which we started, and in terms of which we constructed our FI term. Consequently, even in the most general possible case, we conclude that there is no self-consistent supercurrent superfield in the chiral formalism which can correspond directly to the FI term and thereby exhibit manifest $R_{5}$-invariance.

These results do, however, illustrate one important theme: the breaking of $U(1)_{\mathrm{FI}}$ gauge invariance in the chiral formalism is directly related to the breaking of $R_{5^{-}}$ symmetry. We have already seen this connection at the level of the chiral compensators in Sect. 4.2: because the chiral compensator $\Sigma$ carries both an $R_{5}$-charge and a $U(1)_{\text {FI }}$ charge, both symmetries are broken simultaneously when the chiral compensator is given a VEV. However, this connection is also apparent from our supercurrent expressions. Identifying Eq. (4.15) as our supercurrent supermultiplet in the chiral formalism, we find that $j_{\mu}^{(5)} \sim A_{\mu}$, and on the basis of this result (and 
other results of a similar nature) we see that the FI supercurrent supermultiplet fails to be $U(1)_{\mathrm{FI}}$ gauge invariant [25]. This makes sense, since the chiral formalism is well known to explicitly break $U(1)_{\mathrm{FI}}$ in the presence of a non-zero FI term (see, e.g., Refs. [8, 12]). However, we now see from the first equation in Eq. (4.35) that if we could also demand consistency with $R_{5}$-current conservation, we would simultaneously be imposing the constraint $\partial^{\mu} A_{\mu}=0-i . e$, a compensating gauge choice. At an algebraic level, the constraint equations in Eq. (4.35) would have the net effect of correctly restoring $R_{5}$-current conservation, as they must, while simultaneously eliminating the $A_{\mu}$-dependence within $j_{\mu}^{(5)}$ which was the source of the $U(1)_{\mathrm{FI}}$ gauge non-invariance of the theory. Thus, we see that the issue of the gauge non-invariance of the FI supercurrent supermultiplet in the chiral formalism is a direct consequence of fact that the chiral formalism also breaks manifest $R_{5}$-symmetry. Indeed, both of these features emerge only because the supercurrent supermultiplet in Eq. (4.15) corresponds not to our original globally supersymmetric theory in Eq. (3.24) (in which both $R_{5}$-symmetry and $U(1)_{\mathrm{FI}}$ gauge invariance are preserved), but to its chirallycompensated cousin (in which both symmetries are broken).

There is yet another way in which we can demonstrate our inability to consistently demand $R_{5}$-current conservation in the chiral formalism, as would be required if Eq. (4.15) were the FI supercurrent supermultiplet which directly corresponds to the theory in Eq. (3.24) (as opposed to its chirally-compensated cousin). Using the equations of motion (3.25) for this theory, we find from Eq. (4.15) that

$$
\begin{aligned}
\bar{D}^{\dot{\alpha}} J_{\alpha \dot{\alpha}} & =-\frac{1}{3} \xi \bar{D}^{2} D_{\alpha} V+\frac{4}{3} \xi\left(\sigma^{\mu} \bar{D}\right)_{\alpha} \partial_{\mu} V \\
& =-\frac{1}{3} \xi D_{\alpha} \bar{D}^{2} V .
\end{aligned}
$$

Moreover, if this supercurrent were to conserve $R_{5}$-symmetry, we have already seen that $V$ would have to be truncated according to Eq. (4.35) - i.e., $V$ would have to become a linear multiplet, up to Kähler transformations which do not affect the physics. However, if $V$ were to become a linear multiplet (up to Kähler transformations), then by definition $\bar{D}^{2} V=0$ (up to Kähler transformations), and consequently we see from Eq. (4.36) that $\bar{D}^{\dot{\alpha}} J_{\alpha \dot{\alpha}}=0$ (up to Kähler transformations which do not affect the physics). This in turn implies that our theory would actually have to exhibit not only an unbroken $R_{5}$-symmetry (as required), but also a full unbroken superconformal symmetry. This, of course, is inconsistent with the fact that the FI term introduces a mass scale into the theory.

This is in fact a general phenomenon: a supercurrent supermultiplet in the chiral formalism can exhibit manifest $R_{5}$-current conservation only when the theory itself is superconformal. Indeed, consulting Table 2, we see that $R_{5}$-current conservation in the chiral formalism requires that $\operatorname{Im} F=0$, where $F$ is the auxiliary field within the chiral multiplet $S$. However, the irreducibility of $S$ with respect to supersymmetry transformations implies that we cannot set $\operatorname{Im} F=0$ without setting $S=0$. This 
will only happen for a superconformal theory.

Thus, to summarize: Eq. (4.15) does not represent the supercurrent supermultiplet corresponding to Eq. (3.24). As we have shown in Sect. 3.4, any potential supercurrent superfield $J_{\alpha \dot{\alpha}}$ corresponding to the FI term must preserve $R_{5}$-symmetry, since $j_{\mu}^{(5)}$-conservation is guaranteed by the Noether theorem, and no possible improvement terms beyond the Noether result can change such a critical piece of physics. However, Eq. (4.15) does represent the supercurrent supermultiplet of a theory which is a close cousin to that in Eq. (3.24), namely its chirally-compensated counterpart. Not surprisingly, this counterpart theory has broken $R_{5}$-invariance and broken $U(1)_{\mathrm{FI}}$ invariance as a result of the assignment of a non-zero VEV to the chiral compensator field $\Sigma$. This, then, is the source of the $U(1)_{\mathrm{FI}}$ gauge non-invariance of the result in Eq. (4.15), and the source of its ensuing implications.

\subsection{Evading the proof?}

In Sect. 4.4, we demonstrated that one cannot employ the chiral formalism in order to derive a supercurrent superfield corresponding to an $R_{5}$-invariant theory such as the FI theory in Eq. (3.18). Indeed, we showed that imposing $R_{5}$-invariance on the final result yields the constraint equations in Eqs. (4.35), and these transcend the equations of motion for $V$. As we discussed, these constraint equations essentially imply that $V$ must be a linear multiplet, up to Kähler transformations which do not change the physics. In general, such a truncation of $V$ is unacceptable, as it does not embody the full set of symmetries of the action.

However, if our Lagrangian also were to contain other terms (in addition to the FI term) which modify the equations of motion for $V$ so that they would now be consistent with the constraint equations in Eq. (4.35), no inconsistency would result. In such a case, a fully consistent, $R_{5}$-conserving FI supercurrent supermultiplet $\Xi_{\alpha \dot{\alpha}}^{(C)}$ could potentially be constructed.

One major clue towards a possible choice for such extra Lagrangian terms comes from the fact that the resulting modified equations of motion for $V$, along with their supersymmetric extensions, would have to include the gauge non-invariant constraint that $\partial_{\mu} A^{\mu}=0$. Thus, any suitable extra Lagrangian term must break the $U(1)_{\mathrm{FI}}$ gauge invariance of the theory.

The obvious choice is to introduce a supersymmetric mass term $m^{2} V^{2}$ into the Lagrangian. In other words, let us now take our theory to be given by

$$
\mathcal{L}=\frac{1}{4}\left(\left.W^{\alpha} W_{\alpha}\right|_{\theta \theta}+\left.\bar{W}_{\dot{\alpha}} \bar{W}^{\dot{\alpha}}\right|_{\overline{\theta \theta}}\right)+\left.m^{2} V^{2}\right|_{\theta \theta \overline{\theta \theta}}+\left.2 \xi V\right|_{\theta \theta \overline{\theta \theta}} .
$$

We then find that the new equations of motion take the general form

$$
\begin{aligned}
\partial_{\mu} F^{\mu \nu} & =m^{2} A^{\nu} \\
m^{2} M & =m^{2} N=0 \\
m^{2} \lambda_{\alpha} & =-i m^{2}\left(\sigma^{\mu} \partial_{\mu} \bar{\chi}\right)_{\alpha}
\end{aligned}
$$




$$
\begin{aligned}
m^{2} D & =-m^{2} \square C \\
D & =-m^{2} C-\xi \\
m^{2} \chi_{\alpha} & =-i\left(\sigma^{\mu} \partial_{\mu} \bar{\lambda}\right)_{\alpha},
\end{aligned}
$$

along with the Bianchi identity $\partial_{\mu} \tilde{F}^{\mu \nu}=0$, where $\tilde{F}^{\mu \nu} \equiv \frac{i}{2} \epsilon^{\mu \nu \lambda \sigma} F_{\lambda \sigma}$. For $m=0$, of course, these equations reduce to the equations of motion of the usual gauge-invariant $U(1)_{\text {FI }}$ theory with an FI term. However, for $m \neq 0$, these equations become

$$
\begin{aligned}
\partial_{\mu} F^{\mu \nu} & =m^{2} A^{\nu} \\
M & =N=0 \\
\lambda_{\alpha} & =-i\left(\sigma^{\mu} \partial_{\mu} \bar{\chi}\right)_{\alpha} \\
D & =-\square C \\
D & =-m^{2} C-\xi \\
m^{2} \chi_{\alpha} & =-i\left(\sigma^{\mu} \partial_{\mu} \bar{\lambda}\right)_{\alpha} .
\end{aligned}
$$

Indeed, combining these equations, we now have

$$
\square^{\prime} C-\xi=\square^{\prime} \chi_{\alpha}=\square^{\prime} A_{\mu}=\square^{\prime} \lambda_{\alpha}=\square^{\prime} D=M=N=0,
$$

where $\square^{\prime} \equiv \square-m^{2}$. Thus, for $m \neq 0$, we see that the equations of motion themselves reduce $V$ to a linear multiplet, whereupon we also have the constraint $\partial_{\mu} A^{\mu}=0$ as a consequence of the supersymmetry algebra.

It is clear that these equations are a subset of those in Eq. (4.35). Thus, for $m \neq 0$, Eq. (4.35) is automatically satisfied and there is no inconsistency in taking $T_{S} \sim V$. In other words, in the presence of a supersymmetric mass for the $U(1)_{\mathrm{FI}}$ gauge field, there is no fundamental obstruction to constructing an $R_{5}$-conserving FI contribution $\Xi_{\alpha \dot{\alpha}}^{(C)}$ to the supercurrent supermultiplet, even within the chiral formalism.

However, this observation begs the question: to what extent can we claim that such a broken- $U(1)_{\mathrm{FI}}$ theory really has an FI term? After all, the Kähler potential for this theory can be written in the form

$$
K=m^{2} V^{2}+2 \xi V,
$$

but thanks to the mass $m$, we are always free to define a shifted vector superfield $V^{\prime}$,

$$
V^{\prime} \equiv V+\frac{\xi}{m^{2}},
$$

in terms of which the Kähler potential now takes the form

$$
K=m^{2}\left(V^{\prime}\right)^{2}-\frac{\xi^{2}}{m^{2}} .
$$

Note that both the shift in Eq. (4.42) and the overall constant in Eq. (4.43) have no physical effects on a theory with global supersymmetry. Even in a theory with 
local supersymmetry, these implications of taking $m \neq 0$ still hold, even though these shifts will have other physical effects. As a consequence, it makes perfect sense that this is the one case in which there is no obstruction to building an FI supercurrent supermultiplet $\Xi_{\alpha \dot{\alpha}}$ : indeed, this is the one case in which our theory really has no FI term at all.

Thus, our central result still stands: true FI terms do not lead to self-consistent $R_{5}$-preserving contributions $\Xi_{\alpha \dot{\alpha}}^{(C)}$ for supercurrent supermultiplets. Only when the theory has a "fake" FI term, as discussed above, does such a corresponding supercurrent supermultiplet exist.

It is important to note that even in this case, this still does not yield a total supercurrent supermultiplet $J_{\alpha \dot{\alpha}}^{(C)}$ which exhibits an unbroken $R_{5}$-symmetry in the chiral formalism. The reason is that the addition of the $m^{2} V^{2}$ term into the Lagrangian induces a further, $m$-dependent contribution to the supercurrent supermultiplet, and this further contribution will necessarily break $R_{5}$-symmetry again. Thus, while it is possible to achieve a partial success in which $\Xi_{\alpha \dot{\alpha}}^{(C)}$ exhibits an unbroken $R_{5}$-symmetry, use of the chiral formalism guarantees that this can never be a property of the total supercurrent supermultiplet as a whole.

\section{Analysis in the linear formalism}

As we have seen in Sect. 4, the difficulties that arise in theories with FI terms in the chiral formalism arise essentially because the chirally-compensated $D$-term action in Eq. (4.11) is not gauge invariant under $U(1)_{\mathrm{FI}}$ transformations unless the chiral compensators $\Sigma$ and $\bar{\Sigma}$ are charged under $U(1)_{\mathrm{FI}}$. Furthermore, since the chiral compensators also carry non-zero $R_{5}$-charges, we see that $R_{5}$-invariance is always broken in the chiral formalism, regardless of whether it was preserved in the globallysupersymmetric version of the theory. As a consequence of this artificial breaking of the $R_{5}$ symmetry, we found that the FI supercurrent supermultiplet $\Xi_{\alpha \dot{\alpha}}^{(C)}$ in the chiral formalism is not only non-vanishing in theories with FI terms, but also fails to be gauge invariant.

Unfortunately, many of these conclusions hinged on the structure of the chiral formalism itself. It is therefore unclear to what extent these inconsistencies are general truths about FI terms in supergravity, or merely artifacts of the conformalcompensator formalism employed. Indeed, as discussed in the Introduction, there exist other, alternative formulations of supergravity with different sets of conformal compensators in which the action of the conformally-compensated theory remains invariant under $U(1)_{\mathrm{FI}}$ transformations. It is therefore important to understand whether the primary conclusion of Sect. 4 - namely that theories with non-zero FI terms must exhibit additional global symmetries - holds in such alternative formalisms as well. If not, there would then be no problem in coupling any theory with an FI term to supergravity, so long as this theory admitted a description in such a formalism. 
Our primary goal, then, is to understand the extent to which the conclusions of Sect. 4 and their implications for FI terms are modified by a change of framework. In this section, we will therefore re-examine the issues involved with coupling FI terms to supergravity using an alternate framework: the so-called "linear-compensator" or "new minimal" formalism of Ref. [10]. By its very nature, this formalism manifestly preserves both $R_{5}$-symmetry and $U(1)_{\text {FI }}$ gauge invariance; hence the gauge-invariance issues that arise in the chiral formalism due to the spurious breaking of $R_{5}$-invariance by the compensator fields will not arise here.

We begin this section with a brief review of the linear formalism. We then give a proof that in this formalism, no additional FI contribution $\Xi_{\alpha \dot{\alpha}}^{(L)}$ to the supercurrent supermultiplet can possibly exist. We shall then demonstrate how this same result can be understood through the linear compensator formalism, and finally discuss one possible case in which this result might seem to be altered.

\subsection{Linear compensator formalism: General outline}

In the linear formalism, just as in the chiral formalism, one introduces a set of conformal compensator fields, the role of which is to restore superconformal invariance to the theory in question. Thus, in this way, the resulting theory may be successfully be coupled to conformal supergravity. The fundamental ingredients of the linear formalism are:

- a linear compensator multiplet $L$, with Weyl weight $w_{L}=2$ and vanishing $R_{5}$-charge;

- a pair of chiral compensator multiplets $\Sigma_{L}, \bar{\Sigma}_{L}$, with Weyl weights $w_{\Sigma_{L}}=w_{\bar{\Sigma}_{L}}=$ 1 and $R_{5}$-charges $\pm 2 / 3$ respectively; and

- a new local $U(1)$ symmetry, henceforth denoted $U(1)_{L}$, under which

$$
\Sigma_{L} \rightarrow \Sigma_{L} e^{-i \Lambda_{L}}, \quad \bar{\Sigma}_{L} \rightarrow \bar{\Sigma}_{L} e^{i \bar{\Lambda}_{L}},
$$

where $\Lambda_{L}$ is a chiral superfield parameter. The remaining fields in the theory will be assumed neutral under $U(1)_{L}$ transformations. This $U(1)_{L}$ symmetry plays a crucial role in determining the structure of the action in the linear formalism, as we shall soon see. Note that even though this symmetry is local, we do not introduce any corresponding gauge bosons. This is therefore a local $U(1)$ symmetry of the action, but not a fully dynamical gauge symmetry. We will discuss this symmetry further below.

We begin by discussing how an arbitrary Kähler potential may be made superconformal in this context. Just as in the case of the chiral formalism, let us assume that we may write our Kähler potential in the form $K=K\left(\Phi, \Phi^{\dagger}, V\right)=\sum_{n} K_{n}$ where $K_{n}$ has Weyl weight $n$ and vanishing $R_{5}$-charge. We can then restore scale invariance 
to the theory by multiplying each term in $K$ by an appropriate power of $L$ so as to define a new quantity with vanishing Weyl weight:

$$
\widetilde{K}_{L}\left(\Phi_{i}, \Phi_{i}^{\dagger}, V\right) \equiv \sum_{n}\left(\frac{L}{3 M_{P}^{2}}\right)^{-n / 2} K_{n} .
$$

In terms of this new quantity $\widetilde{K}_{L}$, the Kähler potential $\widehat{K}$ of our conformally compensated theory is then given by

$$
\begin{aligned}
\widehat{K} & \equiv L \ln \left[\frac{L}{\Sigma_{L} \bar{\Sigma}_{L}} \exp \left(\frac{\widetilde{K}_{L}\left(\Phi_{i}, \Phi_{i}^{\dagger}\right)}{3 M_{P}^{2}}\right)\right] \\
& =L \ln \left(\frac{L}{\Sigma_{L} \bar{\Sigma}_{L}}\right)+L \frac{\widetilde{K}_{L}\left(\Phi_{i}, \Phi_{i}^{\dagger}\right)}{3 M_{P}^{2}}
\end{aligned}
$$

whereupon the corresponding $D$-term Lagrangian of the compensated theory is

$$
\mathcal{L}=\int d^{4} \theta L \ln \left[\frac{L}{\Sigma_{L} \bar{\Sigma}_{L}} \exp \left(\frac{\widetilde{K}_{L}\left(\Phi_{i}, \Phi_{i}^{\dagger}\right)}{3 M_{P}^{2}}\right)\right] .
$$

As in the chiral formalism, we can then reproduce our original Lagrangian by setting our compensator fields to fixed values, i.e.,

$$
L \rightarrow 3 M_{P}^{2}, \quad \Sigma_{L}, \bar{\Sigma}_{L} \rightarrow \sqrt{3} M_{P},
$$

and then taking the $M_{P} \rightarrow \infty$ limit. Indeed, following this procedure, we see that $\widehat{K} \rightarrow K$ directly.

Several comments are in order. First, it should be noted that this is already quite different from the analogous situation in the chiral formalism. In the chiral formalism, we did not find $\widehat{K} \rightarrow K$ upon "freezing" our compensator fields and taking $M_{P} \rightarrow \infty$; indeed, this only occurred upon integrating over $d^{4} \theta$. Moreover, even after doing this integration, we still did not precisely reproduce our original theory in the chiral formalism; we only reproduced its overall algebraic form. Indeed, in the final version, all of the non-trivial Weyl weights and $R_{5}$-charges were ultimately stripped from the fields in question. By contrast, in the linear formalism, we have not tampered with our fundamental matter fields $\Phi$ at all. Thus, upon fixing our compensator fields to fixed values and taking the $M_{P} \rightarrow \infty$ limit, our original theory is reproduced exactly. In other words, there is no "hysteresis" effect that emerges upon introducing our compensators and then freezing them to fixed values.

Second, we note that the Kähler portion of the compensated Lagrangian is invariant not only under Weyl rescalings and $R_{5}$-symmetries, but also under local $U(1)_{L}$ transformations. Under the $U(1)_{L}$ transformation in Eq. (5.1), we see from Eq. (5.3) that

$$
\widehat{K} \rightarrow \widehat{K}+i L\left(\Lambda_{L}-\bar{\Lambda}_{L}\right)
$$


However, it may easily be shown as a mathematical identity that for any linear superfield $L$ and chiral superfield $\Lambda_{L}$, the quantity

$$
\int d^{4} \theta L \Lambda_{L}
$$

is a total derivative. Specifically, in component form, we find

$$
\begin{aligned}
\delta \mathcal{L} & =\int d^{4} \theta L \Lambda_{L} \\
& =-\frac{1}{4}\left(\phi_{L} \square C-C \square \phi_{L}\right)-\frac{i}{2} A^{\mu} \partial_{\mu} \phi_{L}-\frac{1}{\sqrt{2}}\left(\overline{\chi \sigma^{\mu}} \partial_{\mu} \psi_{L}-\psi_{L} \sigma^{\mu} \partial_{\mu} \bar{\chi}\right) \\
& =\partial_{\mu}\left[\frac{1}{4}\left(C \partial^{\mu} \phi_{L}-\phi_{L} \partial^{\mu} C\right)+\frac{1}{\sqrt{2}}\left(\psi \sigma^{\mu} \bar{\chi}\right)-\frac{i}{2} A^{\mu} \phi_{L}\right]
\end{aligned}
$$

where $\left\{C, \chi_{\alpha}, \bar{\chi}_{\dot{\alpha}}, A^{\mu}\right\}$ and $\left\{\phi_{L}, \psi_{L}, F_{L}\right\}$ are the component fields within $L$ and $\Lambda_{L}$ respectively. Thus, the action of our theory is invariant under $U(1)_{L}$ transformations. Note that $\Lambda_{L}$ and $\bar{\Lambda}_{L}$ have been treated as fields in this analysis rather than as constant parameters; consequently the full symmetry of the action is local rather than global. This remains true even though no gauge multiplet corresponding to the $U(1)_{L}$ symmetry has been introduced.

Finally, we note that the Kähler portion of our action is also invariant under $U(1)_{\mathrm{FI}}$ gauge transformations. The logic is similar to the previous case. Under $U(1)_{\mathrm{FI}}$ transformations of the form in Eq. (4.19), we see that

$$
\widehat{K} \rightarrow \widehat{K}+\frac{2 i \xi}{3 M_{P}^{2}} L(\Lambda-\bar{\Lambda})
$$

Under $\int d^{4} \theta$ integration, this too yields a total divergence.

We now consider the superpotential $W(\Phi)$ in the linear formalism. It turns out that the form that the superpotential can take in the linear formalism is far more restricted than it was in the chiral formalism. This is due to the presence of the additional $U(1)_{L}$ symmetry, which we are demanding be a symmetry of our superconformal compensated theory. Because the chiral compensator fields $\Sigma_{L}$ and $\bar{\Sigma}_{L}$ carry non-zero $U(1)_{L}$ charges in addition to their Weyl weights and $R_{5}$-charges, these fields can no longer compensate for explicit breakings of Weyl or $R_{5}$-invariance in the superpotential in the same way that $\Sigma$ and $\bar{\Sigma}$ were previously able to do in the chiral formalism. Indeed, while $\Sigma$ and $\bar{\Sigma}$ in the chiral formalism were able to "soak up" unwanted Weyl weights and $R_{5}$-charges from the different terms of our superpotential, we see that $\Sigma_{L}$ and $\bar{\Sigma}_{L}$ can no longer do the same thing without simultaneously breaking $U(1)_{L}$.

As a result, we see that our superpotential $W(\Phi)$ cannot be appropriately compensated in the linear formalism in order to build a fully superconformal theory unless it was already $R_{5}$-invariant and Weyl-invariant to begin with. Thus, we conclude that 
the linear compensator formalism can be only used to produce a superconformal, compensated theory from an original theory which is already $R_{5}$-invariant and whose superpotential is also already conformally (Weyl) invariant [10]. However, if a theory satisfies these criteria, we may still define an alternative set of rescaled matter fields $\widetilde{\Phi}_{L i}, \widetilde{\Phi}_{L i}^{\dagger}$ through the relations

$$
\Phi_{i}=\left(\frac{\Sigma_{L}}{\sqrt{3} M_{P}}\right)^{w_{i}} \widetilde{\Phi}_{L i}, \quad \Phi_{i}^{\dagger}=\left(\frac{\bar{\Sigma}_{L}}{\sqrt{3} M_{P}}\right)^{w_{i}} \widetilde{\Phi}_{L i}^{\dagger}
$$

Like $\widetilde{\Phi}_{i}$ and $\widetilde{\Phi}_{i}^{\dagger}$ in the chiral formalism, these new fields will have vanishing Weyl weight. Furthermore, as we have seen, the Weyl weight and $R_{5}$-charge of any chiral supermultiplet must be related through Eq. (4.2). Thus $\widetilde{\Phi}_{L i}$ and $\widetilde{\Phi}_{L i}^{\dagger}$ also have vanishing $R_{5}$-charge. They are, however, charged under $U(1)_{L}$.

Note that although $\Sigma_{L}$ and $\bar{\Sigma}_{L}$ have non-trivial $R_{5}$-charges, the act of setting these compensator multiplets to the constant values in Eq. (5.5) will not affect the $R_{5^{-}}$ invariance of the theory. This is because these compensator fields appear in Eq. (5.3) only in the $R_{5}$-invariant combination $\Sigma_{L} \bar{\Sigma}_{L}$. Thus, once we fix our compensators to the fixed values in Eq. (5.5), we see that "frozen" theory will continue to preserve $R_{5}$-invariance, in sharp contrast to what happens in the chiral formalism. This, in turn, implies that $\partial^{\mu} j_{\mu}^{(5)}=0$, and that the conservation equation for the supercurrent supermultiplet takes the form $\bar{D}^{\dot{\alpha}} J_{\alpha \dot{\alpha}}^{(L)}=L_{\alpha}$, as discussed in Sect. 3. Indeed, as we shall show explicitly in Sect. 5.3, the variations of $\Sigma_{L}$ and $\bar{\Sigma}_{L}-$ unlike those of $\Sigma$ and $\bar{\Sigma}$ in the chiral formalism — do not contribute to $j_{\mu}^{(5)}$.

\subsection{Deriving the FI supercurrent supermultiplet in the linear formal- ism: A proof of the non-existence of an FI contribution $\Xi_{\alpha \dot{\alpha}}$ to the supercurrent supermultiplet}

We now turn to address the FI contribution $\Xi_{\alpha \dot{\alpha}}^{(L)}$ to the supercurrent supermultiplet within the linear formalism. As we shall prove, the only possible solution consistent with the symmetries of the theory is $\Xi_{\alpha \dot{\alpha}}^{(L)}=0$. Clearly, this result differs from the corresponding, non-zero result for $\Xi_{\alpha \dot{\alpha}}^{(C)}$ in Eq. (4.14), which was derived using the chiral formalism. This then provides graphic illustration that the form of the supercurrent supermultiplet in theories with non-zero FI terms is highly formalismdependent.

In order to show that any additional contribution $\Xi_{\alpha \dot{\alpha}}^{(L)}$ to the supercurrent supermultiplet must vanish in the linear formalism, we begin by noting that amongst all of the possible terms that may appear in a supersymmetric Lagrangian with a $U(1)$ gauge symmetry, the FI term is unique in that it simultaneously exhibits three properties:

- First, because the FI coefficient has a mass dimension, the FI term introduces a mass scale (or equivalently a cosmological constant) into the theory and consequently breaks superconformal invariance. 
- Second, because both $D$ and $C$ are neutral under $R_{5}$-symmetries, the FI term preserves $R_{5}$-symmetry. This was explicitly verified in Sect. 3.4 using the Noether procedure, and this must remain true - regardless of the addition of any possible improvement terms - for any supercurrent supermultiplet which is to be directly associated with the FI term. This $R_{5}$-invariance should be manifest for any supermultiplet constructed within the linear formalism.

- Finally, unlike kinetic terms or mass terms which are quadratic in the fundamental fields of the theory - and likewise unlike superpotential terms which are often cubic or higher in the fundamental fields - the FI term is linear in the fundamental fields.

As we shall now prove, no term which has these three properties simultaneously can yield a non-vanishing contribution $\Xi_{\alpha \dot{\alpha}}^{(L)}$ to the supercurrent supermultiplet of the theory.

Our proof proceeds as follows. As we discussed in Sect. 3, any theory which breaks superconformal invariance while preserving $R_{5}$-symmetry must give rise to a supercurrent supermultiplet within the linear formalism whose lowest components $\left(C_{\mu}, \chi_{\mu \alpha}, \hat{T}_{\nu \mu}\right)$ satisfy the reduced supersymmetry algebra in Eq. (3.15). However, given this reduced supersymmetry algebra, it is possible to consider two successive supersymmetry transformations of magnitudes $\eta$ and $\epsilon$ respectively and thereby derive a self-consistency constraint on the single field $\chi_{\mu}$ :

$$
\begin{aligned}
\delta_{\epsilon} \delta_{\eta} \chi_{\mu \alpha} & =\left(\sigma^{\nu} \bar{\eta}\right)_{\alpha}\left(\partial_{\nu} \delta_{\epsilon} C_{\mu}+i \delta_{\epsilon} \hat{T}_{\nu \mu}\right) \\
& =i\left(\sigma^{\nu} \bar{\eta}\right)_{\alpha}\left[-\epsilon \sigma^{\rho} \bar{\sigma}_{\nu}\left(\partial_{\rho} \chi_{\mu}\right)+\overline{\epsilon \sigma}_{\nu} \sigma^{\rho}\left(\partial_{\rho} \bar{\chi}_{\mu}\right)\right] \\
& =-2 i\left(\epsilon \sigma^{\nu} \bar{\eta}\right)\left(\partial_{\nu} \chi_{\mu \alpha}\right)+2 i(\overline{\epsilon \eta})\left(\sigma^{\nu} \partial_{\nu} \bar{\chi}_{\mu}\right)_{\alpha}
\end{aligned}
$$

This single constraint equation then governs what possible solutions for $\chi_{\mu}$ might exist: any $\chi_{\mu}$ which fails to satisfy this constraint equation cannot possibly be a component of the appropriate supercurrent supermultiplet. By contrast, any $\chi_{\mu}$ which satisfies this equation might or might not lead an appropriate self-consistent supercurrent supermultiplet; in such cases, it would still remain to verify that appropriate solutions for $C_{\mu}$ and $\hat{T}_{\nu \mu}$ in Eq. (3.15) also exist.

One critical feature of the constraint equation in Eq. (5.11) is that its right side is independent of $\eta$, depending only on $\bar{\eta}$. As we see from Eq. (3.15), this is a direct consequence of the fact that $M_{\mu}=N_{\mu}=0$. As discussed in Sect. 3.3, this in turn is a general feature of supercurrent supermultiplets in theories with unbroken $R_{5}$-symmetry.

In general, there are potentially many forms for $\chi_{\mu \alpha}$ which will satisfy Eq. (5.11). However, because we are focusing on the specific case of an FI term $\Xi_{\alpha \dot{\alpha}}^{(L)}$, we see that $\chi_{\mu \alpha}$ must be linear in the fields that appear as components of our original supermultiplet $V$ in Eq. (2.1). The Lorentz vectorial/spinorial nature of $\chi_{\mu \alpha}$, along 
with elementary dimensional analysis, then require that $\chi_{\mu \alpha}$ can at most take the form

$$
\chi_{\alpha}^{\mu}=X\left(\sigma^{\mu} \bar{\lambda}\right)_{\alpha}+Y \partial^{\mu} \chi_{\alpha}+Z\left(\sigma^{\mu \nu} \partial_{\nu} \chi\right)_{\alpha},
$$

where $X, Y$, and $Z$ are unknown (generally complex) coefficients. Inserting this ansatz into Eq. (5.11), it is then possible to obtain self-consistency constraints on the coefficients $X, Y$, and $Z$.

As a first step, it is fairly easy to show that we must have $Y=Z=0$. Indeed, taking Eq. (5.12) for $\chi_{\alpha}^{\mu}$ and evaluating the double-variation $\delta_{\epsilon} \delta_{\eta} \chi_{\alpha}^{\mu}$, we obtain an expression whose $\eta$-dependent terms are given by

$$
\left.\delta_{\epsilon} \delta_{\eta} \chi_{\alpha}^{\mu}\right|_{\eta}=\left[\left(Y g^{\mu \nu}+Z \sigma^{\mu \nu}\right) \eta\right]_{\alpha}\left(2 i \overline{\epsilon \sigma}^{\rho} \partial_{\nu} \partial_{\rho} \chi+2 \bar{\epsilon} \partial_{\nu} \bar{\lambda}\right)
$$

However, we see from Eq. (5.11) that all $\eta$-dependent terms must cancel. Since $g^{\mu \nu}$ and $\sigma^{\mu \nu}$ are respectively even and odd under exchange of their Lorentz indices, this requires that each term vanish separately in the coefficient of Eq. (5.13). We thus have $Y=Z=0$.

Given this, we can now proceed to test whether the sole remaining possible term

$$
\chi_{\alpha}^{\mu} \stackrel{?}{=} X\left(\sigma^{\mu} \bar{\lambda}\right)_{\alpha}=X \sigma_{\alpha \dot{\alpha}}^{\mu} \bar{\lambda}^{\dot{\alpha}}
$$

satisfies the constraint equation in Eq. (5.11). Evaluating the double-variation of this expression directly in terms of the underlying fields in the theory and focusing first on the terms which are holomorphic in $\epsilon$, we find

$$
\begin{aligned}
\left.\delta_{\epsilon} \delta_{\eta} \bar{\lambda}_{\dot{\alpha}}\right|_{\epsilon} & =i \bar{\eta}_{\dot{\alpha}}\left(\epsilon \sigma^{\mu} \partial_{\mu} \bar{\lambda}\right)+\frac{i}{2}\left(\overline{\eta \sigma}^{\nu} \sigma^{\mu}\right)_{\dot{\alpha}}\left(\epsilon \sigma_{\nu} \partial_{\mu} \bar{\lambda}\right)-\frac{i}{2}\left(\overline{\eta \sigma}^{\nu} \sigma^{\mu}\right)_{\dot{\alpha}}\left(\epsilon \sigma_{\mu} \partial_{\nu} \bar{\lambda}\right) \\
& =i\left[\bar{\eta}_{\dot{\alpha}}\left(\epsilon \sigma^{\mu} \partial_{\mu} \bar{\lambda}\right)+\left(\epsilon \sigma^{\mu}\right)_{\dot{\alpha}}\left(\bar{\eta} \partial_{\mu} \bar{\lambda}\right)-\left(\partial_{\mu} \bar{\lambda}_{\dot{\alpha}}\right)\left(\epsilon \sigma^{\mu} \bar{\eta}\right)\right] \\
& =-2 i \partial_{\mu} \bar{\lambda}_{\dot{\alpha}}\left(\epsilon \sigma^{\mu} \bar{\eta}\right) .
\end{aligned}
$$

In passing to the third line of Eq. (5.15), we have used the hermitian conjugate of the cyclic identity $A_{\alpha}(B C)+B_{\alpha}(C A)+C_{\alpha}(A B)=0$, where $A, B$, and $C$ are all spinors. From Eq. (5.15), it then follows that

$$
\left.\delta_{\epsilon} \delta_{\eta} \chi_{\alpha}^{\mu}\right|_{\epsilon}=-2 i X\left(\sigma^{\mu} \partial_{\nu} \bar{\lambda}\right)_{\alpha}\left(\epsilon \sigma^{\nu} \bar{\eta}\right)=-2 i X\left(\partial_{\nu} \chi_{\alpha}^{\mu}\right)\left(\epsilon \sigma^{\nu} \bar{\eta}\right)
$$

in complete accordance with Eq. (5.11). Thus, the terms which are holomorphic in $\epsilon$ succeed in satisfying the constraint equation (5.11) for any $X$.

However, if we examine the terms which depend on $\bar{\epsilon}$, we find that

$$
\begin{aligned}
\left.\delta_{\epsilon} \delta_{\eta} \bar{\lambda}^{\dot{\alpha}}\right|_{\bar{\epsilon}} & =-i \bar{\eta}^{\dot{\alpha}}\left(\overline{\epsilon \sigma}^{\mu} \partial_{\mu} \lambda\right)+\frac{i}{2} \epsilon^{\dot{\alpha} \dot{\beta}}\left(\overline{\eta \sigma}^{\nu} \sigma^{\mu}\right)_{\dot{\beta}}\left(\overline{\epsilon \sigma}_{\nu} \partial_{\mu} \lambda-\overline{\epsilon \sigma}_{\mu} \partial_{\nu} \lambda\right) \\
& =-i\left[\bar{\eta}^{\dot{\alpha}}\left(\overline{\epsilon \sigma}^{\mu} \partial_{\mu} \lambda\right)-\left(\bar{\sigma}^{\mu} \partial_{\mu} \lambda\right)^{\dot{\alpha}}(\overline{\epsilon \eta})+\bar{\epsilon}^{\dot{\alpha}}\left(\overline{\eta \sigma}^{\mu} \partial_{\mu} \lambda\right)\right] \\
& =2 i\left(\bar{\sigma}^{\mu} \partial_{\mu} \lambda\right)^{\dot{\alpha}}(\overline{\epsilon \eta}),
\end{aligned}
$$


whereupon we find that the double-variation of $\chi_{\alpha}^{\mu}$ in Eq. (5.14) is given by

$$
\left.\delta_{\epsilon} \delta_{\eta} \chi_{\alpha}^{\mu}\right|_{\bar{\epsilon}}=2 i X\left(\sigma^{\mu} \bar{\sigma}^{\nu} \partial_{\nu} \lambda\right)_{\alpha}(\overline{\epsilon \eta})
$$

By contrast, the $\bar{\epsilon}$-dependent terms on the right side of Eq. (5.11) yield

$$
2 i X^{*}\left(\sigma^{\nu} \partial_{\nu} \bar{\chi}^{\mu}\right)_{\alpha}(\overline{\epsilon \eta})=-2 i X^{*}\left(\sigma^{\nu} \bar{\sigma}^{\mu} \partial_{\nu} \lambda\right)_{\alpha}(\overline{\epsilon \eta})
$$

and we see that the expressions in Eqs. (5.18) and (5.19) are unequal. Indeed, we find that Eqs. (5.18) and (5.19) differ by the non-zero quantity

$$
-4\left[(\operatorname{Re} X)\left(i \partial^{\mu} \lambda_{\alpha}\right)+2(\operatorname{Im} X)\left(\sigma^{\mu \nu} \partial_{\nu} \lambda\right)_{\alpha}\right](\overline{\epsilon \eta})
$$

which does not even vanish on-shell. We thus conclude that the ansatz in Eq. (5.14) fails to satisfy the constraint equation in Eq. (5.11), or equivalently that there is only one self-consistent solution for $\chi_{\alpha}^{\mu}$ in Eq. (5.12):

$$
X=Y=Z=0
$$

Thus, we conclude that

$$
\Xi_{\alpha \dot{\alpha}}^{(L)}=0
$$

We stress again that this result does not imply that no supercurrent supermultiplet can ever be constructed for theories in which superconformal symmetry is broken while $R_{5}$-symmetry is preserved. Rather, what we have shown is that this cannot be done using expressions which are linear in the component fields that appear within the vector multiplet $V$ in Eq. (2.1). Such expressions would be required for a potential FI contribution $\Xi_{\alpha \dot{\alpha}}^{(L)}$ to the total supercurrent supermultiplet of any theory containing an FI term.

It is worth emphasizing that our results were derived without use of the equations of motion of the theory. Equations of motion, of course, are the one feature of a theory which connect the different terms in its Lagrangian and thereby allow the presence of one term in the Lagrangian to affect the on-shell appearance of the supercurrent contributions from another term. Therefore, it might seem that use of equations of motion could potentially invalidate the term-by-term approach to calculating the supercurrent supermultiplet which is inherent in our implicit separation of the supercurrent into a non-FI piece and an FI piece. However, our proof of the non-existence of an FI supercurrent supermultiplet $\Xi_{\alpha \dot{\alpha}}^{(L)}$ is intrinsically an off-shell proof: it asserts that there exist no self-consistent solutions for $\left(C_{\mu}, \chi_{\mu \alpha}, \hat{T}_{\nu \mu}\right)$ within $\Xi_{\alpha \dot{\alpha}}^{(L)}$ regardless of the equations of motion. This permits our proof to hold in all generality.

Similarly, our proof is also independent of the precise mapping relations between the supercurrent superfield components $\left(C_{\mu}, \chi_{\mu \alpha}, \hat{T}_{\nu \mu}\right)$ and the supercurrents 
$\left(j_{\mu}^{(5)}, j_{\mu \alpha}, T_{\nu \mu}\right)$. Thus our proof should hold even in formulations in which these mapping relations are modified, as long as the underlying supersymmetry algebra is consistent with the constraint $M_{\mu}=N_{\mu}=0$ which characterizes $R_{5}$-invariant theories.

Finally, we remark that the supercurrent superfield is not a physical object; it can be modified by Kähler transformations and other sorts of unphysical improvement terms. However, since our proof has shown that there exist no consistent solutions for the components of the FI contribution $\Xi_{\alpha \dot{\alpha}}^{(L)}$ to the total supercurrent superfield, this remains true despite the possibility of performing Kähler transformations or adding improvement terms. Indeed, all that was assumed in our proof was the fact that our supercurrent superfield is consistent with unbroken $R_{5}$-symmetry, and this in turn led directly to our constraint equation in Eq. (5.11). Consequently, our proof holds despite the possibility of Kähler transformations and other improvement terms.

Thus, to summarize: in the linear formalism, theories that contain FI terms do not yield corresponding FI contributions $\Xi_{\alpha \dot{\alpha}}^{(L)}$ to their supercurrent supermultiplets. Of course, such theories continue to have $R_{5}$-currents, supercurrents, and energymomentum tensors (each of which can be derived through the Noether procedure and improved in various ways), and in general the FI term contributes to both the supercurrent and the energy-momentum tensor. However, what we have proven is that there is no self-consistent $\xi$-dependent supermultiplet structure that can be associated with these FI contributions. It is this feature which stands in stark contrast to the analogous result in the chiral formalism.

It is important to note that the results of this proof do not necessarily lead to any inconsistency insofar as the total supercurrent supermultiplet for the entire theory in question is concerned, even in the presence of a non-zero FI term. Indeed, we note that applying the mapping relations in Eq. (3.13) to the superfield components in Eq. (3.23) yields results for the Noether currents which encapsulate not only those in Eq. (3.20), but also those in Eq. (3.26) when the equations of motion that are used are those that exist in the presence of an FI term. Indeed, within the linear formalism, this is a general phenomenon for any theory with an FI term: regardless of what superpotential terms might be added to the theory, the presence of a non-zero FI term with coefficient $\xi$ has the net effect of shifting $D$ to $D+\xi$ in the equations of motion, and this shift, when applied to the current contributions frm the $U(1)_{\mathrm{FI}}$ kinetic terms, always automatically generates the extra current contributions given in Eq. (3.26). Thus, we see that within the linear formalism, the supercurrent superfield in Eq. (3.22) encapsulates the correct individual currents regardless of whether or not an FI term is introduced: the appearance of an FI term simply shifts the equations of motion for the $D$-field in such a way that the required extra $\xi$-dependent current contributions are automatically incorporated. 


\subsection{Deriving the supercurrent supermultiplet in the linear formalism: Noether calculation}

In Sect. 5.2, we gave an algebraic proof that no additional contribution $\Xi_{\alpha \dot{\alpha}}^{(L)}$ to the supercurrent supermultiplet exists in the linear formalism. However, it is also possible to understand this result by performing an explicit Noether calculation in the fully compensated superconformal theory, taking into account the variations of the compensator fields, in much the same manner as we did in the chiral formalism in Sect. 4.2. In what follows, we perform such a calculation and show that these compensator fields yield no additional contribution to $j_{\mu}^{(5)}$ in the $M_{P} \rightarrow \infty$ limit, i.e., that $\Xi_{\alpha \dot{\alpha}}^{(L)}=0$. We therefore confirm, this time using the linear compensator formalism, that there is no additional FI contribution $\Xi_{\alpha \dot{\alpha}}^{(L)}$ to the supercurrent supermultiplet.

Let us begin by considering how such an FI supercurrent contribution $\Xi_{\alpha \dot{\alpha}}^{(L)}$ could possibly have arisen. Clearly, since the linear compensator field $L$ carries no $R_{5^{-}}$ charge, its variations will yield no Noether contribution to $j_{\mu}^{(5)}$. However, the variations of $\Sigma_{L}$ and $\bar{\Sigma}_{L}$ could potentially contribute to $j_{\mu}^{(5)}$, as these fields carry non-trivial $R_{5}$-charges.

We now demonstrate that no such contribution arises. Although we could do this through a brute-force calculation as in Sect. 4.2 , it is possible to take a useful shortcut. First, we observe that the variation of the Lagrangian of the compensated theory in Eq. (5.4) with respect to any component field $\zeta$ (or derivative of such a component field) within $\Sigma_{L}$ or $\bar{\Sigma}_{L}$ becomes

$$
\frac{\partial \mathcal{L}}{\partial \zeta}=-\int d^{4} \theta \frac{L}{\Sigma_{L} \bar{\Sigma}_{L}} \frac{\partial\left(\Sigma_{L} \bar{\Sigma}_{L}\right)}{\partial \zeta} \longrightarrow-\int d^{4} \theta \frac{\partial\left(\Sigma_{L} \bar{\Sigma}_{L}\right)}{\partial \zeta}
$$

where we have set $L, \Sigma_{L}$, and $\bar{\Sigma}_{L}$ to their corresponding fixed values in passing to the final expression. However, we recognize that this is nothing but the form that we would be dealing with if we were calculating the Noether contributions to $j_{\mu}^{(5)}$ coming from the Kähler term in the trivial Wess-Zumino model with $K=\Phi \Phi^{\dagger}$. Therefore, up to an overall prefactor, we can borrow the well-known Noether result for the Wess-Zumino model to write

$$
\left.j_{\mu}^{(5)}\right|_{\Sigma, \bar{\Sigma}}=\frac{2 i}{3}\left(\phi_{\Sigma}^{*} \partial_{\mu} \phi_{\Sigma}-\phi_{\Sigma} \partial_{\mu} \phi_{\Sigma}^{*}\right)+\frac{1}{3} \psi_{\Sigma} \sigma_{\mu} \bar{\psi}_{\Sigma}
$$

where $\left\{\phi_{\Sigma}, \psi_{\Sigma}, F_{\Sigma}\right\}$ are the component fields of $\Sigma_{L}$.

The next step is to set the component fields in $\Sigma_{L}$ and $\bar{\Sigma}_{L}$ to their fixed values

$$
\phi_{\Sigma} \rightarrow M_{P}, \quad \psi_{\Sigma}, F_{\Sigma} \rightarrow 0
$$

However, when we do this, we find that their contribution to $j_{\mu}^{(5)}$ in Eq. (5.24) vanishes:

$$
\left.j_{\mu}^{(5)}\right|_{\Sigma, \Sigma} \rightarrow 0
$$


Identifying this as the bottom component of the supercurrent superfield $\Xi_{\alpha \dot{\alpha}}^{(L)}$, we thus once again see that

$$
\Xi_{\alpha \dot{\alpha}}^{(L)}=0
$$

in complete agreement with the results of our proof in Eq. (5.22).

We therefore conclude that $\Sigma_{L}$ and $\bar{\Sigma}_{L}$ do not contribute to $j_{\mu}^{(5)}$ in the way that $\Sigma$ and $\bar{\Sigma}$ do in the chiral formalism. Indeed, $\Xi_{\alpha \dot{\alpha}}^{(L)}=0$, and the supercurrent supermultiplet in the linear formalism remains exactly what it was in the uncompensated theory. As a result, our supercurrent supermultiplet is manifestly $U(1)_{\mathrm{FI}}$ gauge invariant.

\subsection{Evading the proof?}

In Sect. 4.4, within the context of the chiral formalism, we proved that an FI supercurrent supermultiplet $\Xi_{\alpha \dot{\alpha}}^{(C)}$ with conserved $R_{5}$-symmetry does not exist. Indeed, as we discussed in Sect. 4.5, there is only one exception to this result: this occurs if the $U(1)_{\mathrm{FI}}$ gauge boson has a supersymmetric mass $m$, corresponding to the introduction of a mass term $\int d^{4} \theta m^{2} V^{2}$ into the Lagrangian. In this section, we shall explain how the introduction of a mass term also allows us to evade the linear-formalism proof in Sect. 5.2. We shall also explicitly construct the $\xi$-dependent supercurrent supermultiplet $\Xi_{\alpha \dot{\alpha}}^{(L)}$ that results.

Recall that within the context of the linear formalism, we showed that our ansatz for $\chi_{\mu \alpha}$ in Eq. (5.12) can satisfy the constraint equation in Eq. (5.11) only if $X=$ $Y=Z=0$. Indeed, this is the result that emerged when we applied the generic equations of motion corresponding to a massless $U(1)_{\mathrm{FI}}$ gauge boson. However, in evaluating quantities such as $\delta_{\epsilon} \delta_{\eta} \chi_{\mu \alpha}$ in the presence of a mass term, we may now make use of the equations of motion in Eq. (4.39). It is easy to see that this can have a profound effect on our conclusions. For example, we previously found that the $\eta$-dependent terms in Eq. (5.13) do not vanish, as they must for consistency, unless $Y=Z=0$. However, we now see that when the equations of motion in Eq. (4.39) are applied, the final parenthesized factor in Eq. (5.13) vanishes all by itself. We are therefore no longer forced to conclude that $Y=Z=0$.

Thus, we shall now quickly repeat our analysis of the possible solutions to the constraint equation in Eq. (5.11), bearing in mind the new equations of motion in Eq. (4.39). First, we observe that as a result of the equations of motion, we have

$$
\left(\sigma^{\mu \nu} \partial_{\nu} \chi\right)_{\alpha}=\frac{i}{2}\left(\sigma^{\mu} \bar{\lambda}\right)_{\alpha}+\frac{1}{2} \partial^{\mu} \chi_{\alpha}
$$

Thus, even our ansatz in Eq. (5.12) simplifies from three possible terms down to two. We shall therefore take our new ansatz to be

$$
\chi_{\alpha}^{\mu}=X\left(\sigma^{\mu} \bar{\lambda}\right)_{\alpha}+Y \partial^{\mu} \chi_{\alpha}
$$

where $X$ and $Y$ are unknown (generally complex) coefficients. 
We now must evaluate $\delta_{\epsilon} \delta_{\eta} \chi_{\alpha}^{\mu}$, and compare this with the right side of Eq. (5.11). We have already seen above that the $\eta$-dependent terms cancel as a result of the new equations of motion, as they must, so this does not provide any constraint on $X$ or $Y$. Likewise, we may easily verify that the $\epsilon$-dependent terms also match without providing any constraint on $X$ or $Y$. However, rotating our overall phase for $\chi_{\mu \alpha}$ so that $X$ is real (without loss of generality), we find that the $\bar{\epsilon}$-dependent terms do not match unless

$$
\operatorname{Re} X=-\operatorname{Im} Y,
$$

with $\operatorname{Re} Y$ unconstrained. Likewise, demanding that $\partial_{\mu} \chi_{\alpha}^{\mu}=0$ (as appropriate for a supercurrent supermultiplet in the linear formalism), we find upon use of the equations of motion that $\operatorname{Re} Y=0$. Taking $X=\xi$, we therefore find that our solution for $\chi_{\alpha}^{\mu}$ is given by

$$
\chi_{\mu \alpha}=\xi\left(\sigma_{\mu} \bar{\lambda}\right)_{\alpha}-i \xi \partial_{\mu} \chi_{\alpha}=-2 i \xi \sigma_{\mu \nu} \partial^{\nu} \chi_{\alpha},
$$

whereupon it is straightforward to verify through supersymmetry transformations that the remaining two components are given by

$$
C_{\mu}=\xi A_{\mu} \quad \text { and } \quad \hat{T}_{\nu \mu}=\xi\left(g_{\mu \nu} \square-\partial_{\mu} \partial_{\nu}\right) C+i \xi \tilde{F}_{\nu \mu} .
$$

Indeed, with the identifications in Eqs. (5.31) and (5.32), it is straightforward to check that the algebra in Eq. (3.15) closes through the equations of motion in Eq. (4.39).

We conclude, then, that in the presence of a non-zero mass for the $U(1)_{\mathrm{FI}}$ gauge boson, an FI supercurrent supermultiplet $\Xi_{\alpha \dot{\alpha}}^{(L)}$ can indeed exist, and has components

$$
\begin{aligned}
C_{\mu} & =\xi A_{\mu} \\
\chi_{\mu \alpha} & =\xi\left(\sigma_{\mu} \bar{\lambda}\right)_{\alpha}-i \xi \partial_{\mu} \chi_{\alpha}=-2 i \xi \sigma_{\mu \nu} \partial^{\nu} \chi_{\alpha} \\
\hat{T}_{\nu \mu} & =\xi\left(g_{\mu \nu} \square-\partial_{\mu} \partial_{\nu}\right) C+i \xi \tilde{F}_{\nu \mu} .
\end{aligned}
$$

Remarkably, these are nothing but the components of the superfield

$$
\Xi_{\mu}=-\frac{1}{2} \bar{\sigma}_{\mu}^{\dot{\alpha} \alpha} \Xi_{\alpha \dot{\alpha}} \quad \text { where } \quad \Xi_{\alpha \dot{\alpha}}=-\frac{\xi}{2}\left[D_{\alpha}, \bar{D}_{\dot{\alpha}}\right] V,
$$

which has the same structural form as Eq. (4.14). The key difference here, of course, is the fact that this is valid only in the presence of a non-zero mass for the $U(1)_{\mathrm{FI}}$ gauge boson.

\section{Connecting the chiral and linear formalisms}

In Sects. 4 and 5 respectively, we examined the properties of both the action and the supercurrent superfield for theories with FI terms, first in the chiral formalism, then in the linear formalism. We demonstrated that in the former construction, the additional FI contribution $\Xi_{\alpha \dot{\alpha}}^{(C)}$ to the supercurrent superfield $J_{\alpha \dot{\alpha}}^{(C)}$ is given by 
Eq. (4.14), while in the latter, $\Xi_{\alpha \dot{\alpha}}^{(L)}=0$. This result is of critical importance, for it illustrates that the gauge non-invariance of the supercurrent supermultiplet emphasized in Ref. 25] is a by-product of the particular formalism that was used. However, as we have seen, the breaking of $U(1)_{\text {FI }}$ gauge invariance in the chiral formalism leads to a rich local and global symmetry structure for theories with non-zero FI terms, including an inconsistency when attempting to couple such theories to supergravity. It is therefore important to understand the local and global symmetry structure that emerges in cases in which the linear formalism can also be employed - i.e., in theories with an unbroken $R_{5}$-symmetry. This is particularly relevant in the case of theories with FI terms, since FI terms in and of themselves preserve $R_{5}$-symmetry.

We shall begin by outlining a duality relation, first developed in Refs. [14, 15], between the chiral- and linear-compensator formalisms. We shall then use this duality relation to derive a general connection between the two supercurrent supermultiplets $J_{\alpha \dot{\alpha}}^{(C)}$ and $J_{\alpha \dot{\alpha}}^{(L)}$ (or between their FI contributions $\Xi_{\alpha \dot{\alpha}}^{(C)}$ and $\Xi_{\alpha \dot{\alpha}}^{(L)}$ ) that respectively emerge in the two formalisms. This analysis will result in a general condition that a theory must satisfy in order for these two supercurrent supermultiplets to differ. As we will see, most theories with canonical renormalizable Kähler potentials will not satisfy this condition, but theories with non-zero FI terms do. Finally, we shall then turn our attention to the local and global symmetry structure of theories with non-zero FI terms, this time using the linear formalism. As we shall see, many of their properties mirror those of their chiral-formalism counterparts, yet there are some crucial differences.

\subsection{Duality relation between chiral- and linear-compensator formalisms}

It is clear from the results of Sects. 4 and 5 that the chiral- and linear-formalism descriptions of the same uncompensated theory are, in general, distinct theories with distinct supercurrent supermultiplets $J_{\alpha \dot{\alpha}}^{(C)}$ and $J_{\alpha \dot{\alpha}}^{(L)}$. However, it can also be shown that these descriptions are related by a duality transformation [14] in cases in which the original, uncompensated theory is $R_{5}$-invariant. Indeed, this is a general result, valid for any $R_{5}$-invariant theory.

Our method of demonstrating this duality will be quite simple: we shall introduce a new "intermediate" Lagrangian which is in neither the chiral nor linear formalisms, and then demonstrate that from this single intermediate Lagrangian we may obtain either Eq. (4.10) or Eq. (5.4) by substituting in for the appropriate fields (or sets of fields) using the equations of motion. Whether we obtain Eq. (4.10) or Eq. (5.4) depends on which fields are placed on shell in our intermediate Lagrangian. This is indeed a standard technique for demonstrating a duality between two different theories.

We shall begin with a discussion of the Kähler contribution to our "intermediate" 
Lagrangian, which takes the form

$$
\mathcal{L}_{D}=\int d^{4} \theta\left[U \ln \left(\frac{U e^{\widetilde{K}_{U}\left(\Phi_{i}, \Phi_{i}^{\dagger}\right) / 3 M_{P}^{2}}}{\Sigma_{L} \bar{\Sigma}_{L}}\right)-i U(\Omega-\bar{\Omega})\right] .
$$

Here $\Sigma_{L}$ and $\bar{\Sigma}_{L}$ are the same compensator fields introduced in the linear formalism, carrying non-trivial $U(1)_{L}$ charges; $U$ is a real vector superfield, neutral under all $U(1)$ symmetries; $\Omega, \bar{\Omega}$ are a pair of left- and right-chiral hermitian-conjugate superfields; and

$$
\widetilde{K}_{U} \equiv-3 M_{P}^{2}+\sum_{n}\left(\frac{U}{3 M_{P}^{2}}\right)^{-n / 2} K_{n}
$$

is the analogue of the rescaled Kähler potential $\widetilde{K}_{L}$ defined in Eq. (5.2), but with $U$ in place of $L$ and with an additional constant term $-3 M_{P}^{2}$. Here, as in Eq. (4.6), the subscript on $K_{n}$ corresponds to its Weyl weight.

Just as in the linear formalism, we would like the Lagrangian in Eq. (6.1) to exhibit a full $U(1)_{\mathrm{FI}} \times U(1)_{L}$ invariance. Unfortunately, under $U(1)_{\mathrm{FI}} \times U(1)_{L}$ transformations, we find that the first term in Eq. (6.1) leads to the variations

$$
\delta_{\mathrm{FI}} \mathcal{L}_{D} \ni \frac{2 i \xi}{3 M_{P}^{2}} \int d^{2} \theta U(\Lambda-\bar{\Lambda}), \quad \delta_{L} \mathcal{L}_{D} \ni i \int d^{2} \theta U\left(\Lambda_{L}-\bar{\Lambda}_{L}\right)
$$

These variations are no longer total derivatives, as they were in the linear formalism, because $U$ is a vector multiplet rather than a linear multiplet. Consequently, in order to maintain $U(1)_{\mathrm{FI}} \times U(1)_{L}$ invariance, we require that $\Omega$ and $\bar{\Omega}$ in the second term of Eq. (6.1) transform linearly under both of these symmetries so as to cancel Eq. (6.3):

$$
\Omega \rightarrow \Omega+\left(\frac{2 \xi}{3 M_{P}^{2}} \Lambda+\Lambda_{L}\right), \quad \bar{\Omega} \rightarrow \bar{\Omega}+\left(\frac{2 \xi}{3 M_{P}^{2}} \bar{\Lambda}+\bar{\Lambda}_{L}\right)
$$

With this transformation, the Lagrangian in Eq. (6.1) is completely $U(1)_{L} \times U(1)_{\mathrm{FI}}$ invariant.

Our aim will be to demonstrate that we may obtain either Eq. (4.10) or Eq. (5.4) from this Lagrangian by substituting in for $U$ in the former case, and for $\Omega$ and $\bar{\Omega}$ in the latter case, using the equations of motion.

Let us focus first on reproducing the linear case (5.4). In superfield language, the equations of motion for the $\Omega$ and $\bar{\Omega}$ superfields are

$$
\frac{\partial \mathcal{L}_{D}}{\partial \Omega}=\frac{\partial \mathcal{L}_{D}}{\partial \bar{\Omega}}=0
$$

Given the Lagrangian in Eq. (6.1), these equations require not only that $U$ be real, but also that it have a $\theta^{2} \bar{\theta}^{2}$ component which is a total derivative. There is only one 
way in which this can happen: $U$ must take the form of a linear superfield, so that we may write

$$
U=L
$$

Substituting this into Eq. (6.1) then yields

$$
\mathcal{L}_{D}=\int d^{4} \theta\left[L \ln \left(\frac{L e^{\widetilde{K}_{L}\left(\Phi, \Phi_{i}^{\dagger}\right) / 3 M_{P}^{2}}}{\Sigma_{L} \bar{\Sigma}_{L}}\right)-L\right]
$$

Since $\int d^{4} \theta L$ is a total divergence (as can be verified from the final column of Table 2), we see that Eq. (6.7) implies the same physics as Eq. (5.4). In other words, we have successfully reproduced the linear-compensator formalism. Indeed, from this perspective, we see that $\Omega$ and $\bar{\Omega}$ are nothing but superfield Lagrange multipliers which enforce the linearity constraint on the superfield $L$.

We shall now demonstrate that Eq. (6.1) also leads to the chiral-compensator formalism. To do this, we shall return to Eq. (6.1) but now consider the equation of motion for $U$ :

$$
\sum_{n}\left(1-\frac{n}{2}\right)\left(\frac{U}{3 M_{P}^{2}}\right)^{-n / 2} \frac{K_{n}}{3 M_{P}^{2}}+\ln \left(\frac{U}{\Sigma_{L} \bar{\Sigma}_{L}}\right)=i(\Omega-\bar{\Omega}) .
$$

For arbitrary Kähler potentials, it is impossible to solve this equation for $U$ exactly. However, we may make a simplifying (and ultimately temporary) assumption that our Kähler potential takes the form

$$
K\left(\Phi_{i}, \Phi, V\right)=K_{0}\left(\Phi_{i}, \Phi_{i}^{\dagger}, V\right)+K_{2}\left(\Phi_{i}, \Phi_{i}^{\dagger}, V\right)
$$

Note that this is not a particularly restrictive assumption, as many theories of theoretical and phenomenological interest frequently take this simplified form. For example, any theory comprising a set of matter fields $\Phi_{i}$ with canonical kinetic terms which are charged under a $U(1)$ gauge group with a non-zero FI term will have $K_{2}=\Phi_{i} e^{-Q_{i} V} \Phi_{i}^{\dagger}$ and $K_{0}=2 \xi V$. Later, we shall generalize our duality argument to any Kähler potential of arbitrary form.

With the assumption in Eq. (6.9), we find that Eq. (6.8) reduces to the form

$$
\frac{K_{0}\left(\Phi_{i}, \Phi_{i}^{\dagger}\right)}{3 M_{P}^{2}}+\ln \left(\frac{U}{\Sigma_{L} \bar{\Sigma}_{L}}\right)=i(\Omega-\bar{\Omega}) .
$$

Solving this for $U$ then yields

$$
U=\Sigma_{L} \bar{\Sigma}_{L} \exp [i(\Omega-\bar{\Omega})] \exp \left[-\frac{K_{0}\left(\Phi_{i}, \bar{\Phi}_{i}\right)}{3 M_{P}^{2}}\right] .
$$

Defining

$$
\Sigma \equiv \Sigma_{L} e^{i \Omega}, \quad \bar{\Sigma} \equiv \bar{\Sigma}_{L} e^{-i \bar{\Omega}}
$$


we now see that $\Sigma$ and $\bar{\Sigma}$ appearing in these relations can be identified with the compensators $\Sigma$ and $\bar{\Sigma}$ of the chiral formalism. In particular, as a result of Eq. (6.4), we see that these $\Sigma, \bar{\Sigma}$ fields carry exactly the same non-trivial $U(1)_{\mathrm{FI}}$ charges as they do in the chiral formalism, even though the previous $\Sigma_{L}, \bar{\Sigma}_{L}$ fields were $U(1)_{\mathrm{FI}^{-}}$ neutral. On the other hand, we see that the the new fields $\Sigma, \bar{\Sigma}$ are $U(1)_{L}$-neutral, while the previous $\Sigma_{L}, \bar{\Sigma}_{L}$ fields were $U(1)_{L}$-charged. Thus, we see that the passage from $\Sigma_{L}, \bar{\Sigma}_{L}$ to $\Sigma, \bar{\Sigma}$ essentially trades $U(1)_{L}$ charges for $U(1)_{\mathrm{FI}}$ charges.

Substituting Eq. (6.11) into Eq. (6.1), we then obtain

$$
\begin{aligned}
\mathcal{L}_{D} & =\int d^{4} \theta\left(-U+K_{2}\right) \\
& =\int d^{4} \theta\left[-\Sigma \bar{\Sigma} \exp \left(-\frac{K_{0}}{3 M_{P}^{2}}\right)+K_{2}\right] \\
& =\int d^{4} \theta\left[-\Sigma \bar{\Sigma}\left(1-\frac{K_{0}}{3 M_{P}^{2}}-\frac{3 M_{P}^{2}}{\Sigma \bar{\Sigma}} \frac{K_{2}}{3 M_{P}^{2}}\right)+\mathcal{O}\left(\frac{K_{n}^{2}}{9 M_{P}^{4}}\right)\right] \\
& =\int d^{4} \theta\left[-\Sigma \bar{\Sigma}\left(1-\frac{\widetilde{K}}{3 M_{P}^{2}}\right)+\mathcal{O}\left(\frac{K_{n}^{2}}{9 M_{P}^{4}}\right)\right] \\
& =\int d^{4} \theta\left[-\Sigma \bar{\Sigma} \exp \left(-\frac{\widetilde{K}}{3 M_{P}^{2}}\right)+\mathcal{O}\left(\frac{K_{n}^{2}}{9 M_{P}^{4}}\right)\right]
\end{aligned}
$$

where we have assumed $K_{n} \ll 3 M_{P}^{2}$ and identified $\widetilde{K} \equiv K_{0}+3 M_{P}^{2} K_{2} /(\Sigma \bar{\Sigma})$ in passing to the fourth line, where $\widetilde{K}$ is defined in Eq. (4.6). Thus, up to higher-order terms which vanish in the $M_{P} \rightarrow \infty$ limit, we successfully recover the chiral-formalism expression in the first line of Eq. (4.10).

This result holds for Kähler potentials of the form in Eq. (6.9). However, it actually holds more generally. Recall that for general Kähler potentials, the equation of motion for $U$ is given in Eq. (6.8), or equivalently

$$
U=\Sigma \bar{\Sigma} \exp \left[-\sum_{n}\left(1-\frac{n}{2}\right)\left(\frac{U}{3 M_{P}^{2}}\right)^{-n / 2} \frac{K_{n}}{3 M_{P}^{2}}\right] .
$$

In general, we cannot solve for $U$ in this relation explicitly, as was possible for the simplified case above. However, we can insert this solution into Eq. (6.1) to obtain

$$
\begin{aligned}
\mathcal{L}_{D} & =\int d^{4} \theta\left[U \ln \left(\frac{U}{\Sigma \bar{\Sigma}}\right)-U+\sum_{n}\left(\frac{U}{3 M_{P}^{2}}\right)^{1-n / 2} K_{n}\right] \\
& =\int d^{4} \theta\left[-\sum_{n} U\left(1-\frac{n}{2}\right)\left(\frac{U}{3 M_{P}^{2}}\right)^{-n / 2} \frac{K_{n}}{3 M_{P}^{2}}-U+\sum_{n}\left(\frac{U}{3 M_{P}^{2}}\right)^{1-n / 2} K_{n}\right] \\
& =\int d^{4} \theta\left[\sum_{n}\left(\frac{n}{2}\right)\left(\frac{U}{3 M_{P}^{2}}\right)^{1-n / 2} K_{n}-U\right] .
\end{aligned}
$$


Thus far, we have made no approximations. However, once again taking $K_{n} \ll 3 M_{P}^{2}$ and using Eq. (6.14) to iteratively substitute $U \sim \Sigma \bar{\Sigma}$ at higher orders, we find that Eq. (6.15) yields

$$
\begin{aligned}
\mathcal{L}_{D}= & \int d^{4} \theta\left[\sum_{n}\left(\frac{n}{2}\right)\left(\frac{\Sigma \bar{\Sigma}}{3 M_{P}^{2}}\right)^{1-n / 2} K_{n}-\Sigma \bar{\Sigma}\right. \\
& \left.\quad+\sum_{n}\left(1-\frac{n}{2}\right)\left(\frac{\Sigma \bar{\Sigma}}{3 M_{P}^{2}}\right)^{1-n / 2} K_{n}+\mathcal{O}\left(\frac{K_{n}^{2}}{9 M_{P}^{4}}\right)\right] \\
= & \int d^{4} \theta\left[\sum_{n}\left(\frac{\Sigma \bar{\Sigma}}{3 M_{P}^{2}}\right)^{1-n / 2} K_{n}-\Sigma \bar{\Sigma}+\mathcal{O}\left(\frac{K_{n}^{2}}{9 M_{P}^{4}}\right)\right] \\
= & \int d^{4} \theta\left[-\Sigma \bar{\Sigma} \exp \left(-\frac{\widetilde{K}}{3 M_{P}^{2}}\right)+\mathcal{O}\left(\frac{K_{n}^{2}}{9 M_{P}^{4}}\right)\right]
\end{aligned}
$$

where we have identified $\widetilde{K}=\sum_{n}\left(\Sigma \bar{\Sigma} / 3 M_{P}^{2}\right)^{-n / 2} K_{n}$. Once again, we see that this is identical to the chiral-formalism Lagrangian in the $M_{P} \rightarrow \infty$ limit.

Our discussion thus far has focused on the contributions to the action coming from the Kähler potential, where we have shown that the chiral- and linear-compensator formalisms are essentially related through a duality. Indeed, as we have seen, this "duality" is nothing but a superfield-level Legendre transformation [14, 15]. However, it remains to verify that we can likewise relate the $F$-term contributions coming from a superpotential $W\left(\Phi_{i}\right)$ between the two formalisms. Fortunately, this is quite simple to do, and we shall find that the two contributions are related for all cases in which the superpotential of the original, uncompensated theory has an $R$-symmetry [14].

In order to understand this, let us consider a theory written in the chiral formalism, with an arbitrary superpotential $\widehat{W} \sim \Sigma^{3} \widetilde{W}$. To obtain the corresponding expression in the linear formalism, we may rewrite this expression in terms of $\Sigma_{L}$ by using Eq. (6.12):

$$
\widehat{W}=\Sigma^{3} \widetilde{W}=e^{-3 i \Omega} \Sigma_{L}^{3} \widetilde{W} .
$$

In order to cancel the $\Omega$-dependent phase in this expression, we must be able to absorb this phase through the redefinitions of the chiral matter fields $\Phi_{i}$ given in Eq. (4.3). However, this will only happen if the sum of the $R$-charges of the fields $\Phi_{i}$ appearing in each term in the superpotential $W\left(\Phi_{i}\right)$ of the linear-formalism theory is equal to 2. This requirement is, of course, nothing but the statement that the superpotential of the original, uncompensated theory must be $R$-invariant. Since this is also a necessary condition for the linear-compensator formalism to be valid, this result proves that any theory which may be described using the linear formalism also has a dual description in the chiral formalism. Of course, the converse is not true in general [14].

Likewise, in order to establish the relationship between $U(1)_{\text {FI }}$ gauge transformations in the two dual pictures, we recall from Eq. (6.4) that the Lagrange-multiplier 
superfields $\Omega$ and $\bar{\Omega}$ are required to transform non-trivially under $U(1)_{\text {FI }}$ gauge transformations in order to keep Eq. (6.1) gauge invariant. As a consequence, the field redefinitions in Eq. (6.12) result in $\Sigma$ and $\Sigma$ acquiring $U(1)_{\mathrm{FI}}$ charges equal to $\pm 2 \xi / 3 M_{P}^{2}$, just as they do in the chiral formalism.

Given this discussion, it is straightforward to see how all of this relates to theories containing non-zero FI terms. We have already shown in Sect. 4, through an analysis involving the chiral formalism, that any theory involving an FI term must possess a global $R$-symmetry. Moreover, in order to admit a description in the linear formalism, it is necessary that a theory possess an unbroken $R_{5}$-symmetry. Consequently, any theory that can be described in the linear formalism satisfies the criteria for the duality to hold. Thus, we conclude that any globally-supersymmetric theory which satisfies the consistency conditions for having FI term can be conformally compensated using the linear formalism as well.

\subsection{Duality relation between supercurrent supermultiplets}

We have seen that any theory which admits a description in the linear formalism (and which therefore has an unbroken $R_{5}$-invariance) has a dual description in the chiral formalism. Given this, we now discuss the general relationship between the supercurrent superfields $J_{\alpha \dot{\alpha}}^{(L)}$ and $J_{\alpha \dot{\alpha}}^{(C)}$ in these two dual theories. We also derive a relationship between the quantities $L_{\alpha}$ and $D_{\alpha} S$ in their respective conservation laws. In this way, we shall essentially be demonstrating that the supercurrentsupermultiplet conservation law (3.7) for a theory with a linear multiplet of anomalies can be "traded" for a conservation law (3.6) involving a chiral multiplet of anomalies through a modification of the supercurrent superfield. While this result is already known [19, 7], our demonstration of this relationship will be derived directly from a strict Noether calculation, using the duality between the chiral and linear formalisms outlined in Sect. 6.1. We shall also discuss the direct implications of these results for theories with non-vanishing FI terms, and show that theories with non-vanishing FI terms are essentially unique amongst theories with renormalizable Kähler potentials in having supercurrent superfields $J_{\alpha \dot{\alpha}}^{(L)}$ and $J_{\alpha \dot{\alpha}}^{(C)}$ which actually differ.

Rather than perform direct superfield calculations, our procedure will be to focus

on deriving contributions to the $R_{5}$-current $j_{\mu}^{(5)}$ in both formalisms. As discussed in Sect. 4.2, this current may be calculated through the Noether procedure, even in the chiral formalism, because we are working within the framework of the compensated theories in which superconformal invariance is preserved. We then "freeze" our compensator fields in order to derive our final expressions for $j_{\mu}^{(5)}$ in each theory. As a final step, we then recognize these results as the bottom components of supercurrent superfields, and thereby promote these results to full superfield expressions.

First, we recall that within the frameworks of our two different formalisms, there are only certain terms within the Kähler portions of the corresponding Lagrangians which contribute to non-zero $R_{5}$-currents after the relevant compensator fields are 
frozen. These Lagrangian terms are

$$
\begin{aligned}
& \text { chiral : } & \mathcal{L} & =\int d^{4} \theta\left[\frac{\Sigma \bar{\Sigma}}{3 M_{P}^{2}} K\left(\widetilde{\Phi}_{i}, \widetilde{\Phi}_{i}^{\dagger}, V\right)\right] \\
& \text { linear : } & \mathcal{L} & =\int d^{4} \theta\left[\frac{L}{3 M_{P}^{2}} \widetilde{K}_{L}\left(\Phi_{i}, \Phi_{i}^{\dagger}, V\right)\right]
\end{aligned}
$$

where the superfields $\widetilde{\Phi}_{i}$ in the chiral formalism are defined in Eq. (4.3) and where $\widetilde{K}_{L}$ in the linear formalism is defined in Eq. (5.2). Note that only the chiral compensator fields $\Sigma, \bar{\Sigma}$ and the original superfields $\Phi_{i}, \Phi_{i}^{\dagger}$ carry non-vanishing $R_{5}$-charges; by contrast, the linear compensator field $L$ and the rescaled superfields $\widetilde{\Phi}_{i}, \widetilde{\Phi}_{i}^{\dagger}$ do not carry $R_{5}$-charge. This implies that there are two independent reasons why the corresponding $R_{5}$-currents $j_{\mu}^{(5)}$ of these two theories might differ:

- There will be contributions from the $R_{5}$-variations of the compensator fields $\Sigma, \bar{\Sigma}$ in the chiral formalism which are not present in the linear formalism. In complete analogy with Eq. (4.13), which was derived for $K=2 \xi V$, we find that these contributions take the form

$$
j_{\mu}^{(5)}=-\frac{2}{3} K_{\mu}\left(\widetilde{\Phi}_{i}, \widetilde{\Phi}_{i}^{\dagger}, V\right)
$$

where $K_{\mu}$ is the coefficient of $-\theta \sigma^{\mu} \bar{\theta}$ within the vector superfield $K\left(\widetilde{\Phi}_{i}, \widetilde{\Phi}_{i}^{\dagger}, V\right)$. Promoting this contribution to a superfield expression, we have

$$
J_{\alpha \dot{\alpha}}=\frac{1}{3}\left[D_{\alpha}, \bar{D}_{\dot{\alpha}}\right] K\left(\widetilde{\Phi}_{i}, \widetilde{\Phi}_{i}^{\dagger}\right) .
$$

- There will also be differences in the variations of the matter fields $\Phi_{i}$ in the linear formalism versus $\widetilde{\Phi}_{i}$ in the chiral formalism. Specifically, the difference in the overall $R_{5}$-charge of these superfields changes the $R_{5}$-charges of their individual field components, and thereby alters the way in which these individual field components contribute to $j_{\mu}^{(5)}$.

As an example of how these combined modifications affect the supercurrent superfield, let us begin by considering a simple case: a Wess-Zumino model in which the Kähler potential takes the minimal form $K=\Phi_{i}^{\dagger} e^{V} \Phi_{i}$ and all of the matter fields are assigned $R_{5}$-charge $R_{i}^{(5)}=2 / 3$. Given this Kähler potential, we find that Eq. (6.19) takes the form

$$
\frac{2 i}{3}\left(\widetilde{\phi}_{i}^{*} \partial_{\mu} \widetilde{\phi}_{i}-\widetilde{\phi}_{i} \partial_{\mu} \widetilde{\phi}_{i}^{*}\right)-\frac{2}{3}\left(\widetilde{\psi}_{i} \sigma_{\mu} \widetilde{\bar{\psi}}_{i}\right)
$$

where $\widetilde{\phi}$ and $\widetilde{\psi}$ are respectively the lowest and next-lowest components of $\widetilde{\Phi}$. By contrast, the results of varying the appropriate matter fields in the two formalisms take the general form

$$
i r_{\phi}\left(\phi_{i}^{*} \partial_{\mu} \phi_{i}-\phi_{i} \partial_{\mu} \phi_{i}^{*}\right)-r_{\psi}\left(\psi_{i} \sigma_{\mu} \bar{\psi}_{i}\right)
$$


where $r_{\phi}$ and $r_{\psi}$ are the $R_{5}$-charges of the appropriate fields $\phi$ and $\psi$ respectively, with $\left(r_{\widetilde{\phi}}, r_{\widetilde{\psi}}\right)=(0,-1)$ for the chiral formalism and $\left(r_{\phi}, r_{\psi}\right)=(2 / 3,-1 / 3)$ for the linear formalism. We therefore find that the total contributions to the $R_{5}$-current $j_{\mu}^{(5)}$ are identical in each formalism, differing only in whether the relevant fields are the original fields or the rescaled fields:

$$
\begin{array}{lll}
\text { chiral : } & j_{\mu}^{(5)}=\frac{2 i}{3}\left(\widetilde{\phi}_{i}^{*} \partial_{\mu} \widetilde{\phi}_{i}-\widetilde{\phi}_{i} \partial_{\mu} \tilde{\phi}_{i}^{*}\right)+\frac{1}{3}\left(\widetilde{\psi}_{i} \sigma_{\mu} \overline{\widetilde{\psi}}_{i}\right) \\
& \text { linear : } & j_{\mu}^{(5)}=\frac{2 i}{3}\left(\phi_{i}^{*} \partial_{\mu} \phi_{i}-\phi_{i} \partial_{\mu} \phi_{i}^{*}\right)+\frac{1}{3}\left(\psi_{i} \sigma_{\mu} \bar{\psi}_{i}\right) .
\end{array}
$$

We thus have

$$
\begin{aligned}
& J_{\alpha \dot{\alpha}}^{(C)}=-D_{\alpha} \widetilde{\Phi}_{i} \bar{D}_{\dot{\alpha}} \widetilde{\Phi}_{i}^{\dagger}+\frac{1}{3}\left[D_{\alpha}, \bar{D}_{\dot{\alpha}}\right]\left(\widetilde{\Phi}_{i} \widetilde{\Phi}_{i}^{\dagger}\right) \\
& J_{\alpha \dot{\alpha}}^{(L)}=-D_{\alpha} \Phi_{i} \bar{D}_{\dot{\alpha}} \Phi_{i}^{\dagger}+\frac{1}{3}\left[D_{\alpha}, \bar{D}_{\dot{\alpha}}\right]\left(\Phi_{i} \Phi_{i}^{\dagger}\right)
\end{aligned}
$$

and we see that the functional forms of these two supercurrents in this special case are identical. Finally, after we freeze our compensator fields to their fixed values, we see that $\widetilde{\Phi}_{i} \rightarrow \Phi$. Thus, after freezing, we see that our two supercurrent superfields become truly identical.

Given these results, let us now proceed to consider the general case of an arbitrary Kähler potential $K\left(\Phi, \Phi^{\dagger}, V\right)$ built from matter fields $\Phi_{i}$ with $R_{5}$-charges $R_{i}^{(5)}$ and gauge fields $V$. We shall assume, of course, that this theory preserves $R_{5}$-invariance, so that a description in either the linear or chiral formalism is possible; moreover, we observe that all fields $\Phi_{i}$ must have a common $R_{5}$-charge $R_{\Phi}^{(5)}$ if we are ultimately to obtain a symmetric energy-momentum tensor [7]. In the linear formalism, the supercurrent superfield $J_{\alpha \dot{\alpha}}^{(L)}$ associated with such a theory can then be obtained via a straightforward Noether calculation of $j_{\mu}^{(5)}$, yielding the result

$$
J_{\alpha \dot{\alpha}}^{(L)}=-g_{i \overline{1}} D_{\alpha} \Phi_{i} \bar{D}_{\dot{\alpha}} \Phi_{\overline{1}}^{\dagger}+\frac{R_{\Phi}^{(5)}}{2}\left[D_{\alpha}, \bar{D}_{\dot{\alpha}}\right]\left(\Phi_{i} K_{i}\right)
$$

Here $K_{i} \equiv \partial K / \partial \Phi_{i}$ and the Kähler metric $g_{i \overline{1}}$ is defined in Eq. (4.17). Note that in Eq. (6.25), we have not explicitly indicated the contributions $2 W_{\alpha} e^{V} \bar{W}_{\dot{\alpha}}$ that correspond to each of the gauge-kinetic terms; these terms are the same in each formalism, and will not be discussed further. As a special case, we observe that if our theory has a minimal Kähler potential of the form $K=\Phi_{i}^{\dagger} e^{V} \Phi_{i}$, then $\Phi_{i} K_{i}=K$. With $R_{\Phi}^{(5)}=2 / 3$, the expression in Eq. (6.25) then reduces to that given in Eq. (6.24).

Eq. (6.25) gives the result for $J_{\alpha \dot{\alpha}}^{(L)}$ in the linear formalism. However, as discussed above, the corresponding supermultiplet $J_{\alpha \dot{\alpha}}^{(C)}$ in the chiral formalism differs from $J_{\alpha \dot{\alpha}}^{(L)}$ in two ways: through a shift in the charges of the redefined fields $\widetilde{\Phi}_{i}$ relative to those of the original fields $\Phi_{i}$, and through the addition of the extra compensator-induced 
term in Eq. (6.20). We therefore have

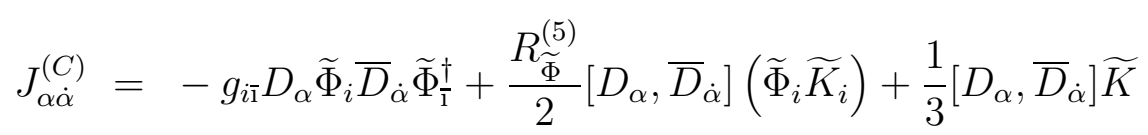

where $\tilde{K} \equiv K\left(\widetilde{\Phi}, \widetilde{\Phi}^{\dagger}, V\right)$. Since $R_{\widetilde{\Phi}}^{(5)}=0$ by construction, and since $\widetilde{\Phi}_{i} \rightarrow \Phi_{i}$ after our compensator fields are frozen, we therefore find $J_{\alpha \dot{\alpha}}^{(C)}$ and $J_{\alpha \dot{\alpha}}^{(L)}$ are connected through the relation

$$
J_{\alpha \dot{\alpha}}^{(C)}=J_{\alpha \dot{\alpha}}^{(L)}+\frac{1}{3}\left[D_{\alpha}, \bar{D}_{\dot{\alpha}}\right]\left(K-\frac{3 R_{\Phi}^{(5)}}{2} \Phi_{i} K_{i}\right) .
$$

This result enables us to determine the general conditions under which $J_{\alpha \dot{\alpha}}^{(C)}$ and $J_{\alpha \dot{\alpha}}^{(L)}$ can differ: this can happen only if there are terms in the Kähler potential for which

$$
\kappa \equiv K-\frac{3 R_{\Phi}^{(5)}}{2} \Phi_{i} K_{i} \neq 0
$$

Clearly, this does not occur for Kähler potentials of the canonical form with $R_{\Phi}^{(5)}=$ $2 / 3$. By contrast, this only happens for non-renormalizable terms involving the matter fields, or situations with $R_{\Phi}^{(5)} \neq 2 / 3$. However, we observe that this also occurs even for a canonical Kähler potential and even with canonical $R_{5}$-charges - in the presence of a non-zero FI term. Indeed, in such a case, we find that $\kappa=2 \xi V \neq 0$. This is then consistent with the results in Eqs. (4.14) and (5.22).

This, then, explains why our results for $\Xi_{\alpha \dot{\alpha}}^{(C)}$ in Sect. 4.2 and $\Xi_{\alpha \dot{\alpha}}^{(L)}$ in Sect. 5.3 are different. Moreover, this also explains why it was possible for the supercurrent supermultiplet in the chiral formalism to break $U(1)_{\text {FI }}$ gauge invariance. Clearly, by construction, the linear formalism preserves $U(1)_{\text {FI }}$ gauge invariance. Thus, we can have a gauge non-invariant $J_{\alpha \dot{\alpha}}^{(C)}$ only when the two supermultiplets are allowed to differ.

Given the result in Eq. (6.27) relating $J_{\alpha \dot{\alpha}}^{(C)}$ and $J_{\alpha \dot{\alpha}}^{(L)}$, it is also possible to derive a relation between the anomalies $L_{\alpha}$ and $D_{\alpha} S$ to which $\bar{D}^{\dot{\alpha}} J_{\alpha \dot{\alpha}}^{(C)}$ and $\bar{D}^{\dot{\alpha}} J_{\alpha \dot{\alpha}}^{(L)}$ are respectively equated. Indeed, writing the linear multiplet in unconstrained form as $L_{\alpha} \equiv \bar{D}^{2} D_{\alpha} T_{L}$ where $T_{L}$ is a vector superfield, it is even possible to solve for $T_{L}$. Starting with the relation

$$
J_{\alpha \dot{\alpha}}^{(C)}=J_{\alpha \dot{\alpha}}^{(L)}+\frac{1}{3}\left[D_{\alpha}, \bar{D}_{\dot{\alpha}}\right] \kappa
$$

where $\kappa$ is defined in Eq. (6.28), we then find

$$
\begin{aligned}
\bar{D}^{\dot{\alpha}} J_{\alpha \dot{\alpha}}^{(C)} & =\bar{D}^{\dot{\alpha}} J_{\alpha \dot{\alpha}}^{(L)}+\frac{1}{3} \bar{D}^{\dot{\alpha}}\left[D_{\alpha}, \bar{D}_{\dot{\alpha}}\right] \kappa \\
& =\bar{D}^{2} D_{\alpha}\left(T_{L}+\kappa\right)-\frac{1}{3} \bar{D}_{\dot{\alpha}} D_{\alpha} \bar{D}^{\dot{\alpha}} \kappa \\
& =D_{\alpha} \bar{D}^{2}\left(T_{L}+\frac{2}{3} \kappa\right)+4 i\left(\sigma^{\mu} \partial_{\mu} \bar{D}\right)_{\alpha}\left(T_{L}+\frac{1}{2} \kappa\right) .
\end{aligned}
$$


However, we know that $\bar{D}^{\dot{\alpha}} J_{\alpha \dot{\alpha}}^{(C)}$ should take the form $D_{\alpha} S$, where $S$ is a left-chiral superfield. We therefore deduce that we must have $T_{L}=-\frac{1}{2} \kappa$, whereupon we conclude that

$$
\begin{aligned}
L_{\alpha} & =\bar{D}^{2} D_{\alpha} T_{L}=-\frac{1}{2} \bar{D}^{2} D_{\alpha} \kappa \\
D_{\alpha} S & =D_{\alpha} \bar{D}^{2}\left(T_{L}+\frac{2}{3} \kappa\right)=-\frac{1}{3} D_{\alpha} \bar{D}^{2} T_{L}=\frac{1}{6} D_{\alpha} \bar{D}^{2} \kappa .
\end{aligned}
$$

\subsection{The symmetry structure of theories with non-zero FI terms in the linear formalism}

In this section, we shall explore the structure of local and global symmetries that appear in theories with non-zero FI terms in the linear formalism. This section therefore serves as the linear-formalism counterpart to Sect. 4.3. We shall begin by describing the general symmetry structure of such theories and the way in which it emerges. We shall then provide an explicit example.

\subsubsection{General symmetry structure}

Thus far in this section, we have demonstrated that a duality exists between the chiral and linear formulations of supergravity. However, while this implies a physical equivalence between the compensated, superconformal theories in these two formulations, it is not yet clear to what extent this equivalence persists after the compensator fields of each formalism are "frozen" in order to break the symmetries of the superconformal group down to those of Poincaré supergravity. Indeed, as we have already discussed in Sects. 4 and 5, we know that certain differences between the formalisms are inevitable after the compensators are frozen. For example, $R_{5}$-symmetry remains a symmetry in the linear formalism, but is necessarily broken in the chiral formalism. Moreover, some of these differences are unique to theories with non-zero FI terms. For example, $U(1)_{\mathrm{FI}}$ gauge symmetry remains a good symmetry within the linear formalism, but is broken in the chiral formalism if a non-zero FI term is present. As a result, the supercurrent supermultiplet $J_{\alpha \dot{\alpha}}^{(L)}$ in theories with FI terms is $U(1)_{\mathrm{FI}}$ gauge-invariant in the linear formalism, whereas the corresponding supermultiplet $J_{\alpha \dot{\alpha}}^{(C)}$ in the chiral formalism is not.

It is therefore important to explore the extent to which the symmetry structures that survive in the linear formalism can be matched to those in the chiral formalism, and to determine whether the issues that arise in coupling theories with non-zero FI terms to supergravity using the chiral formalism also arise in the linear formalism. In this way, we will be testing the extent to which the difficulties in coupling theories with non-zero FI terms to supergravity in the chiral formalism are intrinsic to the FI terms themselves, or are instead primarily features of the formalism that is being used. 
With this goal in mind, we now discuss the symmetry structure of theories that are treated in the linear formalism. Of course, any theory which admits a description in the linear formalism must already possess a global $R_{5}$-symmetry (which will become local once the theory is coupled to supergravity). Moreover, by assumption, our original theory will have an FI symmetry which we will call $U(1)_{\mathrm{FI}}^{\prime}$. While the presence of the non-zero FI term $2 \xi V$ in the Kähler potential breaks Weyl invariance explicitly, the superpotential of the theory is Weyl-invariant by assumption. The theory may possess additional gauge symmetries, of course, but these symmetries will not be affected by the compensator calculus and are therefore not relevant to the present discussion.

The first step in coupling a theory to supergravity in the linear formalism is to introduce the compensator fields $L, \Sigma_{L}$, and $\bar{\Sigma}_{L}$, thereby modifying the $D$-term Lagrangian of the original theory so that it takes the form given in Eq. (5.4). By design, the compensated theory is invariant under both conformal rescalings and $R_{5}$ rotations in addition to special SUSY transformations, implying that it is invariant under full super-Weyl group $U(1)_{\mathrm{SW}}$. Furthermore, unlike the analogous situation in the chiral formalism, the superconformal theory in the linear formalism also directly inherits the full local $U(1)_{\mathrm{FI}}^{\prime}$ gauge symmetry of the original theory thanks to the fact - already discussed below Eq. (5.9) - that $U(1)_{\text {FI }}^{\prime}$ gauge transformations shift the Lagrangian by total derivatives and thus leave the action invariant. Indeed, by suitably covariantizing the partial derivatives appearing in this Lagrangian, it can be shown that all of these symmetries can be gauged without further modification of the action [27, 28. Finally, as discussed in Sect. 5.1, the Lagrangian in Eq. (5.4) also possesses an additional local $U(1)_{L}$ symmetry. However, it should be emphasized that the $U(1)_{L}$ symmetry is not a gauge symmetry, since it has no corresponding gauge bosons. It is nevertheless a local symmetry of the action because - just as with $U(1)_{\mathrm{FI}}^{\prime}$ - an arbitrary local $U(1)_{L}$ transformation causes the Lagrangian of the theory to shift by a total derivative, thereby leaving the action invariant.

In summary, then, we see that our compensated superconformal covariantized theory in the linear formalism will have a local symmetry of the form $U(1)_{\mathrm{FI}}^{\prime} \times$ $U(1)_{\mathrm{SW}} \times U(1)_{L}$. It is, however, to recast this symmetry structure into a slightly different form.

In order to do this, we first observe that the local $U(1)_{L}$ symmetry has the net effect of rendering the additional degrees of freedom in $\Sigma_{L}$ and $\bar{\Sigma}_{L}$ unphysical. Thus, the presence of the $U(1)_{L}$ symmetry allows these degrees of freedom to be gauged away. This can be seen most readily when the Lagrangian is expressed in terms of the original matter fields $\Phi_{i}$, for we then find that $\Sigma_{L}$ and $\bar{\Sigma}_{L}$ are the only fields which transform non-trivially under $U(1)_{L}$.

This implies that any $U(1)$ charges which are assigned to $\Sigma_{L}$ and $\bar{\Sigma}_{L}$ are ultimately unphysical, since their effects under $U(1)$ gauge transformations can be eliminated through a compensating $U(1)_{L}$ transformation. Given this, we are free to define a new FI symmetry — to be denoted $U(1)_{\mathrm{FI}}$ - under which $\Sigma_{L}$ and $\bar{\Sigma}_{L}$ are charged. 
For reasons to become clear shortly, we shall choose $\Sigma_{L}, \bar{\Sigma}_{L}$ to carry the same $U(1)_{\mathrm{FI}}$ charges as the corresponding $\Sigma, \bar{\Sigma}$ fields carry in the chiral formalism, namely

$$
Q_{\Sigma_{L}, \bar{\Sigma}_{L}}= \pm \frac{2 \xi}{3 M_{P}^{2}}
$$

Thus, while our original matter fields $\Phi_{i}$ have $U(1)_{\mathrm{FI}}$ charges which coincide with their $U(1)_{\mathrm{FI}}^{\prime}$ charges, the rescaled matter fields $\widetilde{\Phi}_{L i}$ will not.

One way to think of this shift from the $U(1)_{\mathrm{FI}}^{\prime}$ description to the $U(1)_{\mathrm{FI}}$ description is as a basis change realized through linear combination

$$
U(1)_{\mathrm{FI}} \equiv U(1)_{\mathrm{FI}}^{\prime}-\frac{2 \xi}{3 M_{P}^{2}} U(1)_{L}
$$

In this way, we see that $U(1)_{\mathrm{FI}}^{\prime}$ and $U(1)_{\mathrm{FI}}$ can be freely substituted for each other in the presence of the $U(1)_{L}$ symmetry. However, it is important to stress that this replacement of $U(1)_{\mathrm{FI}}^{\prime}$ by $U(1)_{\mathrm{FI}}$ is not a true change of basis because we should not think of the $U(1)_{\mathrm{FI}}^{\prime}$ gauge boson as somehow picking up a $U(1)_{L}$ charge when being repackaged as a $U(1)_{\mathrm{FI}}$ gauge boson. Rather, we must think of the $U(1)_{\mathrm{FI}}^{\prime}$ gauge boson and the $U(1)_{\mathrm{FI}}$ gauge boson as being one and the same object. As discussed above, this is because the change from $U(1)_{\mathrm{FI}}^{\prime}$ to $U(1)_{\mathrm{FI}}$ has no physical import, and does not alter our theory in any way.

There is, however, one important difference between $U(1)_{\mathrm{FI}}$ and $U(1)_{\mathrm{FI}}^{\prime}$, and this is what ultimately motivates the specific charge choice in Eq. (6.32). We have already remarked that $U(1)_{\mathrm{FI}}^{\prime}$ is a symmetry of the action of our superconformal, compensated theory in the linear formalism; specifically, $U(1)_{\mathrm{FI}}^{\prime}$ transformations cause the Lagrangian of the superconformal theory to shift by total derivatives. However, we expect that a canonical gauge symmetry should not merely leave the action invariant; it should leave the Lagrangian invariant as well. Unfortunately, $U(1)_{\mathrm{FI}}^{\prime}$ does not do this in any manifest way. However, by charging the chiral compensator fields $\Sigma_{L}, \bar{\Sigma}_{L}$ under $U(1)_{\mathrm{FI}}$ as in Eq. (6.32), we see that $U(1)_{\mathrm{FI}}$ is now a full manifest gauge symmetry of the theory, one which leaves the Lagrangian as well as the action invariant. This happens because variations of $V, \Sigma_{L}$, and $\bar{\Sigma}_{L}$ under $U(1)_{\mathrm{FI}}$ transformations now explicitly cancel against each other.

Given these observations, we shall therefore recast the symmetries of our covariantized superconformal theory in the linear formalism as $U(1)_{\mathrm{FI}} \times U(1)_{\mathrm{SW}} \times U(1)_{L}$. All of these symmetries are local; they are also "big" in the sense defined below Eq. (2.4). Likewise, only $U(1)_{\mathrm{Sw}}$ is an $R$-type symmetry. Finally, we again stress that only $U(1)_{\mathrm{FI}}$ and $U(1)_{\mathrm{SW}}$ are gauge symmetries; indeed $U(1)_{L}$, despite being local, lacks a corresponding gauge boson.

Having now redefined the symmetry content of the theory in terms of $U(1)_{\mathrm{FI}}$, we recast the theory in terms of the rescaled matter fields $\widetilde{\Phi}_{L i}$ defined in Eq. (5.10). Since $\Sigma_{L}$ is charged under both $U(1)_{\mathrm{FI}}$ and $U(1)_{L}$, the $\widetilde{\Phi}_{L i}$ fields acquire charges 
under both symmetries due to rescaling. Specifically, we find that the $U(1)_{L}, U(1)_{\mathrm{FI}}$, and $U(1)_{\mathrm{SW}}$ charges of the $\widetilde{\Phi}_{L i}$ matter fields are given by

$$
Q_{\widetilde{\Phi}_{L i}}^{L}=w_{i}, \quad Q_{\widetilde{\Phi}_{L i}}^{\mathrm{FI}}=Q_{\Phi_{i}}^{\mathrm{FI}}-\frac{2 \xi}{3 M_{P}^{2}} w_{i}, \quad Q_{\widetilde{\Phi}_{L i}}^{\mathrm{SW}}=0
$$

while the corresponding charges of the $\Sigma_{L}$ compensator field are given by

$$
Q_{\Sigma_{L}}^{L}=-1, \quad Q_{\Sigma_{L}}^{\mathrm{FI}}=\frac{2 \xi}{3 M_{P}^{2}}, \quad Q_{\Sigma_{L}}^{\mathrm{SW}}=\frac{2}{3} .
$$

However, when we compare these charge assignments to the corresponding ones in the chiral-formalism description of the same theory, we find that they agree exactly. In other words, the charges of $\Sigma_{L}$ and $\widetilde{\Phi}_{L i}$ under $U(1)_{\mathrm{FI}}$ in the linear formalism are precisely those of $\Sigma$ and $\widetilde{\Phi}_{i}$ under $U(1)_{\mathrm{FI}}$ in the chiral formalism. Furthermore, the global $U(1)_{\mathrm{FI}}^{\prime}$ symmetry of the chiral formalism is identical to the $U(1)_{\mathrm{FI}}^{\prime}$ symmetry of the linear formalism, which is simply a linear combination of $U(1)_{L}$ and $U(1)_{\mathrm{FI}}$, as discussed above.

We thus see that the superconformal compensated theories in the chiral and linear formalisms have an almost identical symmetry structure. Both theories are invariant under a gauged $U(1)_{\mathrm{FI}} \times U(1)_{\mathrm{SW}}$ symmetry as well as under an additional global symmetry which can be viewed, depending of the basis of $U(1)$ factors chosen, as either $U(1)_{L}$ or $U(1)_{\mathrm{FI}}$. Indeed, the only difference between the two formalisms is whether this additional symmetry may also be considered local (despite not being gauged) in a particular basis. This, then, is another explicit verification of the duality relation between the chiral and linear formalisms.

As discussed above, this duality relation is only expected to hold at the level of the superconformal theories prior to freezing our compensator fields. Our next step, therefore, is to investigate what happens in the linear formalism once the compensator fields are frozen. In the linear formalism, there are two sets of compensator fields that must be frozen: the chiral compensators $\Sigma_{L}, \bar{\Sigma}_{L}$, and the linear compensator $L$. Freezing each of these has a distinct effect.

Let us first consider freezing the chiral compensators $\Sigma_{L}, \bar{\Sigma}_{L}$. Given the charge assignments in Eq. (6.35), we find that there are always two independent symmetries which survive this freezing (i.e., two independent symmetries under which $\Sigma_{L}, \bar{\Sigma}_{L}$ are uncharged). It is particularly convenient to choose a specific basis for these symmetries:

$$
\begin{aligned}
U(1)_{\mathrm{FI}}^{\prime} & \equiv U(1)_{\mathrm{FI}}+\frac{2 \xi}{3 M_{P}^{2}} U(1)_{L} \\
R_{G} & \equiv \frac{2}{3} U(1)_{L}+U(1)_{\mathrm{SW}}
\end{aligned}
$$

Indeed, if we formally "solve" for $U(1)_{L}$ from the first equation in Eq. (6.36) and substitute this into the second equation, we obtain precisely the definition (4.27) for 
$R_{G}$ in the chiral formalism. Note that $U(1)_{\mathrm{FI}}^{\prime}$ and $R_{G}$ are both local $U(1)$ symmetries which are "big", operating at the superfield level.

The next step is to implement the freezing of the linear compensator $L$. However, $L$ only carries a Weyl charge. Thus, the net effect of freezing $L$ is simply to break the Weyl symmetry of the theory, i.e., to demote the "big" $U(1)_{\mathrm{SW}}$ symmetry to its "little" (Wess-Zumino-gauge) remnant $R_{5}$. This in turn leaves the $U(1)_{\mathrm{FI}}^{\prime}$ symmetry of Eq. (6.36) intact, but demotes $R_{G}$ from "big" to "little" status. Both symmetries remain local, however.

The upshot, then, is that coupling our original globally $R_{5}$-invariant theory to supergravity in the linear formalism results in a frozen theory with two local symmetries: the $U(1)_{\mathrm{FI}}^{\prime}$ gauge symmetry of our original theory, and a new "little" local $R_{G}$ symmetry defined as

$$
R_{G} \equiv \frac{2}{3} U(1)_{L}+R_{5}
$$

However, $R_{G}$ is essentially equivalent to $R_{5}$ as far as the physics of our theory is concerned; indeed, if we work in terms of the original unscaled matter fields $\Phi_{i}$, these symmetries are identical. We thus see that the linear formalism indeed preserves both the original FI gauge symmetry and the original $R_{5}$-symmetry of the theory, as advertised.

We conclude, then, that the process of coupling an $R_{5}$-invariant theory to supergravity in the linear formalism does not alter the original symmetry structure of the theory. Indeed, the only change is that $R_{5}$ is promoted from a global symmetry to a local one whose gauge field is the auxiliary field $b_{\mu}$ of the gravitational multiplet.

\subsubsection{An explicit example}

For a concrete illustration of these ideas, let us revisit the $R_{5}$-invariant toy model with non-zero FI term that was presented in Sect. 4.3.2. We shall now trace how its symmetry structure changes through the various steps in the conformal-compensator calculus, this time in the linear formalism.

We begin by considering the compensated superconformal theory. As discussed above, the linear formalism allows us the option of writing the action for our conformally-compensated theory in terms of either the original matter fields $\Phi_{i}$ of the uncompensated theory, or the rescaled matter fields $\widetilde{\Phi}_{i}$. In the former notation, the Lagrangian for the conformally-compensated theory is given by

$$
\mathcal{L}=\int d^{4} \theta\left[L \ln \left(\frac{L}{\Sigma_{L} \bar{\Sigma}_{L}}\right)+\frac{2 \xi}{3 M_{P}^{2}} L V+K^{\prime}\right]+\left[\int d^{2} \theta W+\text { h.c. }\right]
$$

where $W$ and $K^{\prime}$ are defined in Eqs. (4.25) and (4.26), respectively. Alternatively, 
when written in terms of the compensated fields $\widetilde{\Phi}_{L i}$, this Lagrangian becomes

$$
\mathcal{L}=\int d^{4} \theta\left[L \ln \left(\frac{L}{\Sigma_{L} \bar{\Sigma}_{L}}\right)+\frac{2 \xi}{3 M_{P}^{2}} L V+\frac{\Sigma_{L} \bar{\Sigma}_{L}}{3 M_{P}^{2}} \widetilde{K}_{L}^{\prime}\right]+\left[\int d^{2} \theta\left(\frac{\Sigma_{L}}{\sqrt{3} M_{P}}\right)^{3} \widetilde{W}_{L}+\text { h.c. }\right]
$$

where $\widetilde{K}_{L}^{\prime}$ and $\widetilde{W}_{L}$ take the same form as the corresponding expressions for $K^{\prime}$ and $W$ in Eqs. (4.26) and (4.25), but with $\Phi_{i}$ and $\Phi_{i}^{\dagger}$ replaced everywhere by $\widetilde{\Phi}_{L i}$ and $\widetilde{\Phi}_{L i}^{\dagger}$. This action is invariant under not only $U(1)_{\mathrm{FI}}^{\prime}$ gauge transformations and local superconformal transformations (including local $R_{5}$-rotations), but also under an additional, local $U(1)_{L}$ symmetry (which is a symmetry of the action, but not of the Lagrangian). As discussed above, this $U(1)_{L}$ symmetry allows us complete freedom to redefine $\Sigma_{L}$ and $\Sigma_{L}$. Consequently we may assign charges to these fields under the $U(1)_{\mathrm{FI}}$ gauge symmetry defined in Eq. (6.33) without affecting the physics.

We therefore find that our superconformal theory has the symmetry structure shown in Table 6. Moreover, comparing the symmetries and charge assignments listed in this table to those given in Table 4 for the chirally-compensated theory, we see that the $U(1)_{\mathrm{FI}}$ symmetries of the two theories are identical; that is, the field charges in the linear-formalism description of the theory, written in terms of the rescaled fields $\widetilde{\Phi}_{L i}$, coincide with the field charges of the chiral-formalism description. The $R_{5}$-charges and Weyl-weights in the two theories are likewise identical. Thus,

\begin{tabular}{||c||c|ccc|c||}
\hline \hline Field & $U(1)_{\mathrm{FI}}$ & $U(1)_{\mathrm{SW}}:$ & $R_{5}$ & Weyl & $U(1)_{L}$ \\
\hline \hline$\Phi_{1}$ & +1 & $2 / 3$ & $2 / 3$ & 1 & 0 \\
$\Phi_{2}$ & -1 & $2 / 3$ & $2 / 3$ & 1 & 0 \\
$\Phi_{3}$ & 0 & $2 / 3$ & $2 / 3$ & 1 & 0 \\
\hline$\widetilde{\Phi}_{L 1}$ & $1-2 \xi / 3 M_{P}^{2}$ & 0 & 0 & 0 & +1 \\
$\widetilde{\Phi}_{L 2}$ & $-1-2 \xi / 3 M_{P}^{2}$ & 0 & 0 & 0 & +1 \\
$\widetilde{\Phi}_{L 3}$ & $-2 \xi / 3 M_{P}^{2}$ & 0 & 0 & 0 & +1 \\
\hline$\Sigma_{L}$ & $2 \xi / 3 M_{P}^{2}$ & $2 / 3$ & $2 / 3$ & 1 & -1 \\
$L$ & 0 & $*$ & 0 & 2 & 0 \\
\hline$\lambda_{\alpha}$ & 0 & $*$ & 1 & $3 / 2$ & 0 \\
$\psi_{\mu \alpha}$ & 0 & $*$ & 1 & $3 / 2$ & 0 \\
\hline \hline
\end{tabular}

Table 6: The symmetry structure of the linearly-compensated version of the toy model presented in Sect. 4.3.2. This table may be compared with Table 4, which shows the corresponding symmetry structure within the chiral formalism. Charges are listed for the original matter fields $\Phi_{i}$, their rescaled counterparts $\widetilde{\Phi}_{L i}$, the compensators $\Sigma_{L}$ and $L$, the $U(1)_{\mathrm{FI}}$ gaugino $\lambda_{\alpha}$, and the gravitino $\psi_{\mu \alpha}$. An entry ' $*$ ' indicates that the corresponding field is not a chiral superfield, and therefore its $R_{5}$ - and Weyl-charges cannot be packaged as a chiral charge under $U(1)_{\mathrm{SW}}$. Note that $R_{G}$ is an independent symmetry only when the theory is written in terms of the rescaled fields $\widetilde{\Phi}_{L i}$. 


\begin{tabular}{||c||c|c||}
\hline \hline Field & $U(1)_{\mathrm{FI}}^{\prime}$ & $R_{G}$ \\
\hline \hline$\Phi_{1}$ & +1 & $2 / 3$ \\
$\Phi_{2}$ & -1 & $2 / 3$ \\
$\Phi_{3}$ & 0 & $2 / 3$ \\
\hline$\widetilde{\Phi}_{L 1}$ & +1 & $2 / 3$ \\
$\widetilde{\Phi}_{L 2}$ & -1 & $2 / 3$ \\
$\widetilde{\Phi}_{L 3}$ & 0 & $2 / 3$ \\
\hline$\lambda_{\alpha}$ & 0 & 1 \\
$\psi_{\mu \alpha}$ & 0 & 1 \\
\hline \hline
\end{tabular}

Table 7: The symmetry structure of the final version of our toy model in Table 6, after the compensator fields are "frozen" to their fixed values in the linear formalism. This table may be compared with Table 5, which shows the corresponding symmetry structure within the chiral formalism. Once again, charges are listed for both the original matter fields $\Phi_{i}$ and their rescaled counterparts $\widetilde{\Phi}_{L i}$, as well as for the $U(1)_{\mathrm{FI}}$ gaugino $\lambda_{\alpha}$ and gravitino $\psi_{\mu \alpha}$.

this explicit $R_{5}$-invariant example nicely illustrates the general result of Sect. 6.1, namely that the chiral- and linear-formalism descriptions of such a theory are dual to each other at the level of their conformally-compensated Lagrangians.

We now turn to examine the effects that emerge upon freezing the compensator fields $\Sigma_{L}, \bar{\Sigma}_{L}$, and $L$. As discussed in Sect. 6.3.1, freezing these compensators breaks the local $U(1)_{L} \times U(1)_{\mathrm{FI}} \times U(1)_{\mathrm{SW}}$ invariance of the compensated superconformal theory down to a subgroup comprising a $U(1)_{\text {FI }}^{\prime}$ gauge symmetry and a local $R_{G}$ symmetry. The charge assignments for the various fields of the frozen theory under these symmetries are shown in Table 7.

We see, then, that the FI gauge symmetry of the frozen theory is identical to that of the original, uncompensated theory. This stands in stark contrast to the situation in the chiral formalism in Sect. 4.3.2. But even more importantly, we see that no additional global symmetries are present. Indeed, what remains in addition to the $U(1)_{\mathrm{FI}}^{\prime}$ gauge symmetry is merely a local version of our original $R_{5}$-symmetry, this time with $R_{G}$ playing the role of $R_{5}$.

\section{Discussion}

Although supersymmetric theories with non-zero Fayet-Iliopoulos terms have played a vital role in many areas of particle physics over the past twenty years, history has shown that it is surprisingly difficult to couple such theories to supergravity. While there exists a relatively extensive literature dealing with this and related issues [2-24], recent discussions [25] have sparked renewed interest in this question and added urgency to the task of understanding its resolution. 
In this work, we have sought to clarify the status of Fayet-Iliopoulos terms in supergravity theories. Following the lead of Ref. [25], we have focused on issues pertaining to the supercurrent supermultiplets of globally supersymmetric theories with non-zero FI terms. However, we have also broadened our investigation by studying the overall symmetry properties of such theories, particularly insofar as questions of gauge invariance and $R_{5}$-symmetry conservation are concerned. Towards this end, we have reviewed both the chiral (or "old minimal") and linear (or "new minimal") compensator formalisms of supergravity. This has enabled us to analyze, within each of these formalisms, the salient properties of the actions and supercurrent supermultiplets corresponding to theories containing non-zero FI terms. This also has enabled us to ascertain which features of these theories are intrinsic to FI terms, and which tend to be more closely associated with the formalisms themselves and might therefore be viewed as artifacts of those formalisms. We have also examined the extent to which the well-established duality [14] between the conformally-compensated theories in these two formulations is disturbed after their respective compensator fields are "frozen" in order to break the extraneous symmetries of the superconformal group.

The primary results of our analysis may be summarized as follows.

- First, within the context of a generic globally supersymmetric theory with supercurrent supermultiplet $J_{\alpha \dot{\alpha}}$, we have studied the specific contribution $\Xi_{\alpha \dot{\alpha}}$ within $J_{\alpha \dot{\alpha}}$ that arises due to the possible existence of a non-zero FI term. Specifically, $\Xi_{\alpha \dot{\alpha}}$ may be identified as that part of the overall supercurrent $J_{\alpha \dot{\alpha}}$ which depends explicitly on the Fayet-Iliopoulos coefficient $\xi$ - and which therefore has a linear dependence on the fields associated with the $U(1)_{\mathrm{FI}}$ vector superfield — without use of the equations of motion. Our conclusion is that no self-consistent solutions for $\Xi_{\alpha \dot{\alpha}}$ exist for theories with unbroken $R_{5^{-}}$ symmetry. (Such theories are natural candidates for study, since FI terms by themselves intrinsically preserve $R_{5}$-symmetry.) In the chiral formalism, this happens because one cannot construct a consistent supercurrent supermultiplet $J_{\alpha \dot{\alpha}}^{(C)}$ from the three Noether currents $j_{\mu}^{(5)}, j_{\mu \alpha}$, and $T_{\mu \nu}$ of the flat-space theory. Instead, as we demonstrated in Sect. 4.2, explicit contributions from the compensator fields of this formalism must be included in order to obtain a consistent multiplet, and we have shown in Sect. 4.4 that there is no way in which these contributions can preserve $R_{5}$-symmetry. This peculiar property of FI terms is indicative of the fundamental difficulties which arise in coupling $R_{5}$-invariant theories with non-zero FI terms to supergravity using the chiral formalism. Moreover, in the linear formalism, we have shown in Sect. 5.2 that $\Xi_{\alpha \dot{\alpha}}$ must vanish outright.

- On the other hand, for theories that break $R_{5}$-symmetry, we find that the FI supercurrent contribution $\Xi_{\alpha \dot{\alpha}}$ exists, but necessarily breaks the $U(1)_{\text {FI }}$ gauge symmetry of the original theory. This result is in complete agreement with the results of Ref. 25]. However, as we discuss in Sect. 2, the FI term by itself 
does not break this gauge symmetry. Instead, as we demonstrate in Sect. 4, this breaking of the $U(1)_{\mathrm{FI}}$ symmetry in the presence of a non-zero FI term is entirely an artifact of the chiral-compensator formalism, as originally pointed out in Ref. [8]. Indeed, in the linear formalism, the $U(1)_{\mathrm{FI}}$ gauge symmetry is preserved, even in the presence of a non-zero FI term. However, the linear formalism is not applicable for theories which break $R_{5}$-symmetry.

- Taking a bird's-eye view, these observations suggest that there is a fundamental connection between $R_{5}$-symmetry and $U(1)_{\text {FI }}$ gauge invariance in the presence of a non-zero FI term: either both symmetries are simultaneously preserved (as in the linear formalism), or both are broken (as in the chiral formalism [12]). Using our results from Sect. 5.2, this implies at the level of supercurrent supermultiplets that the only way to have a non-zero FI contribution $\Xi_{\alpha \dot{\alpha}}$ is to break $U(1)_{\mathrm{FI}}$ gauge invariance. This is, of course, precisely what occurs in Ref. [25], but we now see that this is indeed an expected and generic property, forced upon us by the minimal compensator formalisms.

- As we have shown in Sects. 4.4 and 5.4, there is one situation in which we may construct a non-zero FI contribution $\Xi_{\alpha \dot{\alpha}}$ to the supercurrent even when $R_{5^{-}}$ symmetry is conserved: this occurs if our $U(1)_{\text {FI }}$ gauge boson has an explicit supersymmetric mass. Such a situation might arise, e.g., for effective FI theories at lower energy scales. This mass breaks the $U(1)_{\text {FI }}$ gauge symmetry explicitly, but demonstrates that non-zero solutions for $\Xi_{\alpha \dot{\alpha}}$ can exist in such situations. Moreover, to the best of our knowledge, this is the only known situation in which the minimal supergravity formalisms permit $R_{5}$ symmetry to be manifestly preserved while $U(1)_{\text {FI }}$ gauge symmetry is broken in the presence of a non-zero FI term.

- In the chiral formalism, the existence of a non-zero FI term results in the presence of an exact, global symmetry in the conformally-compensated, locally supersymmetric theory at the classical level. This is discussed explicitly in Sect. 4.3. Even after the compensator fields of the theory are "frozen" in order to break superconformal invariance down to Poincaré supersymmetry, this exact global symmetry remains unbroken. This result is in complete agreement with the results of Ref. [25]. Moreover, the symmetries of the frozen theory also include a gauged $R$-symmetry, under which the gravitino and all gauginos in the theory are charged.

- In the linear formalism, the conformally-compensated theory in the presence of a non-zero FI term is dual to the corresponding theory in the chiral formalism. (Indeed, the analogue of the additional $U(1)_{\mathrm{FI}}^{\prime}$ symmetry in the latter is the built-in $U(1)_{L}$ symmetry in the former.) Upon freezing the compensators of the linear formalism, however, the symmetry content of the theory reduces to 
that of the uncompensated theory, the one difference being that $R_{5}$ has become a local symmetry. Consequently, as discussed in Sect. 6.3, all of the symmetries of the final, frozen theory are local.

Several comments about these results are in order.

First, it should be noted that there are many subtleties associated with the process of freezing the conformal compensator fields. Of course, this process is intended to eliminate the extraneous degrees of gauge freedom associated with transformations under the superconformal group (scale invariance, $S$-supersymmetry, etc.) which were not symmetries of the original theory. However, the gauge-fixing conditions imposed in order to break these symmetries often also break other symmetries of that theory which ought to be preserved in the frozen theory. For example, the gauge-fixing conditions break supersymmetry. Likewise, the $R_{5}$-symmetry of an $R_{5^{-}}$ invariant theory will unfortunately be broken in the chiral formalism. In such cases, a set of analogous symmetries can be defined for this theory, the generators of which correspond to linear combinations of the generators of the symmetries of the compensated theory (for an excellent review, see Ref. [13]). Nevertheless, issues of this sort do not directly impact the status of FI terms in supergravity theories, and we have therefore refrained from discussing them in detail in the present work.

Second, it should be emphasized that the analysis presented here has been entirely classical. At the quantum level, the results outlined above are still valid; however, one must be slightly more careful in choosing the compensator formalism one uses in order to couple a given theory to supergravity. In particular, theories which admit a consistent description in the linear formalism at the classical level often do not admit such a description at the quantum level; in models in which the $R_{5}$-symmetry is anomalous, higher-order corrections will induce superconformal-anomaly contributions which are not of the form $L_{\alpha}$, thereby rendering the linear-formalism description invalid [7]. Indeed, this kind of issue is known to affect the anomaly structures of supersymmetric QED and QCD [33, 34]. This poses additional constraints on the applicability of the linear formalism, but these constraints may be overcome. The massless Wess-Zumino model, for example, satisfies such constraints and may be consistently coupled to supergravity using the linear formalism, even at the quantum level.

Third, it is worth mentioning that other supergravity formulations exist in addition to the chiral and linear formalisms discussed here. These include the non-minimal formalism of Ref. [35], as well as a variety of additional possibilities which utilize reducible multiplets as compensators (for a discussion of some of these possibilities, see Ref. [29]). One interesting non-minimal alternative, especially as far as FI terms are concerned, is the vectorial formalism of Ref. [6], in which the compensator fields include a vector multiplet $V$, a chiral superfield $\Sigma_{V}$, and its hermitian conjugate $\bar{\Sigma}_{V}$. This formalism can be viewed as being analogous to the chiral formalism, but with an FI term, whose corresponding gauge field is the vector compensator $V$, already 
incorporated [13]. We will not discuss these possibilities further here, except to note that use of such non-minimal formalisms could offer valuable additional perspectives on many the issues surrounding FI terms in supergravity.

Finally, even within the minimal formalisms we have focused on here, the question of whether theories with primordial non-zero FI terms can be consistently coupled to supergravity is likely to depend on whether $R_{5}$-symmetry is ultimately broken in nature (i.e., on whether the $R_{5}$-symmetry breaking scale is at or near the SUSYbreaking scale). If so, and if the chiral formalism is applicable, then such a coupling to supergravity is likely to be impossible as a result of the extra global symmetry that remains in this formalism, as discussed in Sect. 4.3 and originally pointed out in Ref. [25]. In such a case, if our underlying primordial theory at the highest energy scales is coupled to supergravity, then this theory cannot contain FI terms. As a result, if supersymmetry is realized in nature and broken dynamically at lower energy scales, the mechanism responsible for that breaking will turn out to be predominately $F$-term in nature. However, if $R_{5}$-symmetry is ultimately preserved in nature, then the chiral formalism will no longer be appropriate and the the linear formalism will be more relevant as a description of the corresponding supergravity. In such cases, as discussed in Sect. 6.3, the resulting symmetry structure may well be different.

We conclude, then, that the issue of coupling theories with FI terms to supergravity is indeed a subject with many subtleties and unexpected consequences. Incorporating the FI term into supergravity appears to be uniquely and inherently wedded to the specific formalism employed, which motivates the comparative treatment we have provided here. Moreover, we see that the $R_{5}$-symmetry properties of a theory appear to be closely linked to its FI properties, and both together appear to determine the degree of difficulty with which such a theory can ultimately be coupled to supergravity. Finally, we have seen that the FI contribution $\Xi_{\alpha \dot{\alpha}}$ to the supercurrent $J_{\alpha \dot{\alpha}}$ is a very strange beast: in one formalism it breaks gauge invariance, and in another formalism it doesn't even exist. All of these features together explain why the issue of coupling theories with non-zero FI terms to supergravity has had a long and tortu(r)ous history, and suggest that this topic is likely to have a long and tortu(r)ous future as well.

\section{Acknowledgments}

We are happy to thank Z. Chacko, E. Dudas, S.J. Gates, and especially N. Seiberg for discussions. This work was supported in part by the U.S. Department of Energy under Grant DE-FG02-04ER-41298. The opinions and conclusions expressed here are those of the authors, and do not represent either the Department of Energy or the National Science Foundation. 


\section{References}

[1] P. Fayet and J. Iliopoulos, Phys. Lett. B 51 (1974) 461; P. Fayet, Nucl. Phys. B 90, 104 (1975).

[2] S. Ferrara and B. Zumino, Nucl. Phys. B 87, 207 (1975).

[3] D. Z. Freedman, Phys. Rev. D 15, 1173 (1977).

[4] A. Das, M. Fischler and M. Rocek, Phys. Rev. D 16, 3427 (1977).

[5] K. S. Stelle and P. C. West, Phys. Lett. B 74, 330 (1978); S. Ferrara and P. van Nieuwenhuizen, Phys. Lett. B 74, 333 (1978).

[6] B. de Wit and P. van Nieuwenhuizen, Nucl. Phys. B 139, 216 (1978).

[7] T. E. Clark, O. Piguet and K. Sibold, Nucl. Phys. B 143, 445 (1978).

[8] K. S. Stelle and P. C. West, Nucl. Phys. B 145, 175 (1978).

[9] W. Fischler, H. P. Nilles, J. Polchinski, S. Raby and L. Susskind, Phys. Rev. Lett. 47, 757 (1981).

[10] M. F. Sohnius and P. C. West, Phys. Lett. B 105, 353 (1981).

[11] M. Sohnius and P. C. West, Nucl. Phys. B 198, 493 (1982).

[12] R. Barbieri, S. Ferrara, D. V. Nanopoulos and K. S. Stelle, Phys. Lett. B 113, 219 (1982).

[13] T. Kugo and S. Uehara, Nucl. Phys. B 226, 49 (1983).

[14] S. Ferrara, L. Girardello, T. Kugo and A. Van Proeyen, Nucl. Phys. B 223, 191 (1983); M. T. Grisaru, N. K. Nielsen, W. Siegel and D. Zanon, Nucl. Phys. B 247, 157 (1984).

[15] A. Van Proeyen, Proc. of Karpacz Winter School 1983:0093.

[16] E. Witten, Nucl. Phys. B 268, 79 (1986).

[17] M. A. Shifman and A. I. Vainshtein, Nucl. Phys. B 277, 456 (1986) [Sov. Phys. JETP 64, 428 (1986 ZETFA,91,723-744.1986)].

[18] N. Seiberg, Phys. Lett. B 318, 469 (1993) arXiv:hep-ph/9309335.

[19] T. E. Clark and S. T. Love, Int. J. Mod. Phys. A 11, 2807 (1996) arXiv:hep-th/9506145. 
[20] A. H. Chamseddine and H. K. Dreiner, Nucl. Phys. B 458, 65 (1996) arXiv:hep-ph/9504337;

[21] M. Dine, "Fields, Strings and Duality: TASI 96," eds. C. Efthimiou and B. Green, World Scientific, Singapore (1997).

[22] D. J. Castano, D. Z. Freedman and C. Manuel, Nucl. Phys. B 461, 50 (1996) arXiv:hep-ph/9507397]; P. Binetruy, G. Dvali, R. Kallosh and A. Van Proeyen, Class. Quant. Grav. 21, 3137 (2004) |arXiv:hep-th/0402046]; H. Elvang, D. Z. Freedman and B. Kors, JHEP 0611, 068 (2006) |arXiv:hep-th/0606012].

[23] S. Weinberg, Phys. Rev. Lett. 80, 3702 (1998) arXiv:hep-th/9803099.

[24] R. Kallosh, L. Kofman, A. D. Linde and A. Van Proeyen, Class. Quant. Grav. 17, 4269 (2000) [Erratum-ibid. 21, 5017 (2004)] [arXiv:hep-th/0006179].

[25] Z. Komargodski and N. Seiberg, JHEP 0906, 007 (2009) arXiv:0904.1159 [hepth]].

[26] J. Wess and J. Bagger, "Supersymmetry and supergravity," Princeton University Press, USA (1992).

[27] P. C. West, "Introduction to supersymmetry and supergravity," World Scientific, Singapore (1990).

[28] S. J. Gates, M. T. Grisaru, M. Rocek and W. Siegel, "Superspace, or one thousand and one lessons in supersymmetry," Front. Phys. 58, 1 (1983) arXiv:hep-th/0108200.

[29] T. Kugo and S. Uehara, Nucl. Phys. B 226, 93 (1983).

[30] T. Banks and L. J. Dixon, Nucl. Phys. B 307, 93 (1988).

[31] R. Kallosh, A. D. Linde, D. A. Linde and L. Susskind, Phys. Rev. D 52, 912 (1995) arXiv:hep-th/9502069.

[32] C. P. Burgess, et al, JHEP 0807, 073 (2008) [arXiv:0805.4037 [hep-th]].

[33] T. E. Clark, O. Piguet and K. Sibold, Nucl. Phys. B 159, 1 (1979).

[34] T. E. Clark, O. Piguet and K. Sibold, Nucl. Phys. B 172, 201 (1980).

[35] P. Breitenlohner, Phys. Lett. B 67, 49 (1977). 